UNIVERSIDADE DE SÃO PAULO

INSTITUTO DE FÍSICA E QUÍMICA DE SAOO CARLOS

\title{
O MÉTODO DO CAMPO AUTOCONSISTENTE NO ESTUDO DE PROBLEMAS DE MUITOS CORPOS
}

\author{
VERA BEATRU PRERTAS DE CAMPOS \\ Departamento de Fisica \\ Universidade Federal de São Carlos
}

( Tese Apresentada ao Instituto de Física e Química de São Carlos, USP, para a obtenção do título de "Doutor em Física").

Orientador:

Prof. Dr. Roberto Leal Lobo e Silva Filho

SÃO CARLOS

1976 
UNIVERSIDADE DE SÃO PAULO

INSTITUTO DE FISICA E QUÍMICA DE SÃO CARLOS

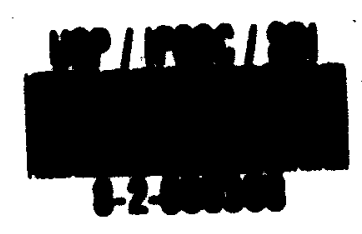

\title{
O MÉTODO DO CAMPO AUTOCONSISTENTE NO ESTUDO DE PROBLEMAS DE MUITOS CORPOS
}

\author{
VERA BEATRIZ FREITAS DE CAMPOS \\ Deportamento de Física \\ Universidade Federal de São Carlos \\ (Tese opresentoda oo Instituto de Firaca \\ e Química de São Carlos, USP, para a \\ obtençōo do título de "Doutor em Física").
}

Orientodor:

Prof. Dr. Roberto Leal Lobo Silva Filho 
Agradeço,

ao prof. Dr. Roberto Leal Lobo e Silva Filho pelo constante apoio, boa vontade e orientação na realização deste trabalho, ao prof. Dr. Oscar Hipólito pelas proveitosas discussões e calaboração, aos colegas e funcionärios do Departamento de Física e da Divisão de Computação da UFSCAR, e do Departamento de Fisica e Ciência dos Ma teriais do IFQSC, USP, que contribuiram direta ou indiretamente pa ra o desenvolvimento deste trabalho,

à Maria Laura pelo excelente trabalho de datilografia.

Finalmeste, agradeço a hospitalidade do IFQSC e o patrocínio da UFSCAR, indispensāveis à realização desta tese. 


\section{APRESENTAÇAOO}

Êste trabalho constitui-se do cálculo de propriedades de sistemas de muitos corpos, em especial líquidos quânticos $\left(T=0^{\circ} \mathrm{K}\right)$, utilizando o método do campo autoconsistente (SCFA). Vamos aplicar a SCFA a sistema quânti cos compostos por partículas neutras ou carregadas (eletrons), estudando a susceptibilidade generalizada do sistema. Assim, obtém-se autoconsistentemen te propriedades como o fator de estrutura, função de correlação dos pares,es pectro de energia das excitações, velocidade do som, etc.

No Capítulo 1 discutimos os conceitos teóricos que serão utilizados,en quanto que o Capítulo 2 é dedicado a uma descrição da SCFA. Singwi e seus colaboradores desenvolveram essa aproximação para estudar o gás de eletrons tridimensional, na região de densidades metálicas, tentando melhorar os resultados até então obtidos por outras aproximações. A hipótese fundamental feita nessa teoria é utilizar a função de correlação dos pares de partículas para expressar a função de distribuição de dyas partículas em termos do produto das funções de distribuição de cada partícula. Então procura-se uma solução autoconsistente para o fator de estrutura e função de correlação dos pares. Este fato implica na obtenção de um potencial efetivo autoconsistente, que representa a interação entre as partículas do gäs.

o Capítulo 3 é dedicado ao estudo de um sistema tridimensional de bosons, comparando-se os resultados obtidos supondo que as partículas possam ser consideradas como esferas totalmente rígidas e introduzindo uma parte atrativa no potencial, a fim de tornā-1o mais semelhante ao potencial real de 
sistemas tipo 4 He. Usando nas expressões obtidas teoricamente os parâme tros correspondentes ao líquido de ${ }^{4} \mathrm{He}$, podemos comparar os resultados com os resultados experimentais obtidos a baixas temperaturas. Verifica-se que a introdução de uma parte atrativa no potencial ocasiona uma melhora nos re sultados, principalmente para a velocidade do som e massa efetiva do roton. Os espectros de energia teórico e experimental apresentam o mesmo comporta mento, com as posições do máximo e do minnimo coincidentes. Entretanto, a altura do primeiro mäximo e do mỉnimo experimentais correspondem a cerca de 50 a $60 \%$ do valor teórico com cada potencial. No caso de potencial com par te atrativa, se supusermos que a massa efetiva do átomo de Hélio no sistemaé cerca de 1.8 vezes maior que a massa do átomo livre, os espectros de energia teórico e experimental vão praticamente coincidir. Então, a velocidade do som tem o valor $s=218 \mathrm{~m} / \mathrm{s}$, bastante mais próximo do valor extrapolado dos resultados experimentais. Podemos também calcular a massa efetiva do átomo de Hélio que corresponderia ao valor experimental para a velocidade do som, obtendo-se $\mathrm{m}_{\mathrm{ef}} \cong 2 \mathrm{~m}_{\mathrm{He}}$. Neste caso, os valores do máximo e do mínimo do es pectro de energia teórico ficam cerca de $8 \%$ maiores que os experimentais.

No Capítulo 4 a aproximação SCFA é aplicada a um sistema de fermions neutros, composto de partỉculas impenetráveis. 0 problema unidimensional foi resolvido completamente. Os resultados para este sistema mostraram uma característica bastante interessante, que é associada ao seu comportamento em função da densidade. Verificou-se que existem duas regiões de densida des com comportamentos bem distintos, tanto para o fator de estrutura e fun ção de correlação dos pares, como para o espectro de energia das excitações elementares e velocidade do som. 0 espectro de energia apresenta desconti 
nuidades quando os modos coletivos encostam no espectro de particula única. Para todas as densidades a primeira descontinuidade ocorre quando $q=\pi / \ell$, que corresponde à inversão do sinal do potencial efetivo autoconsistente. Esse fato é evidente quando se examina a relação de dispersão de um sistema de fermions e verifica-se que a troca de sinal no potencial efetivo deve ne cessariamente corresponder a uma troca de sinal na diferença de energias en tre a excitação coletiva e partỉcula única. Na região de "altas" densida des $(\rho l \geq 0.23)$ o espectro de energia vai apresentar um comportamento tipo "roton", com o minimo de energia acentuando-se quando a densidade cresce. Para densidades mais altas vai aparecer um segundo mínimo, com $q>\pi / \ell$, que, eventualmente, vai corresponder a um zero de energia quando $\rho \ell \sim 0.66$. Es se fato evidencia que o sistema alcançou um estado cristalino, o que é tam bém indicado pelo comportamento fortemente oscilatório da função de correlą ção dos pares nesta região. o comportamento de um sistema unidimensional de fermions considerados como barras rígidas, e o de um sistema de bosons com mesma interação é bastante semelhante, especialmente para baixas densida des, quando os resultados concordam com os resultados exatos de um sistema sem interação.

No Capítulo 5, é feito o estudo de um sistema quasi-unidimensional, onde a condução é feita de maneira unidimensional. Usando a aproximação SCFA, calculamos o fator de estrutura autoconsistente para valores de $x_{\mathrm{s}}=$ $x_{0} / a_{0}$ entre 0.01 e 12 . Com esse resultado obtivemos a função de correlação dos pares em cada caso, comparando-a com o resultado obtido com RPA- que cor responde à aproximação de ordem zero em SCFA. Para $x_{s}=0.01$ os resultados obtidos em ambas as aproximações coincidem, indicando que esse valor corres ponde a uma região de densidades bastante altas. Entretanto, para densida 
des menores, os valores RPA para a função de correlação são inferiores àque les obtidos com SCFA. Para $x_{S}>11$, a função de correlação obtida em RPA é negativa, para pequenas separações de partículas. Entretanto, a aproxima ção SCFA apresenta valores sempre positivos até $x_{s}=12$, que é o valor limi te para o tipo de cálculo desenvolvido. Porém, como estes resultados ainda são bastante positivos, $\mathrm{g}(0) \sim 0.2$, é razoável supor que a aproximação SCFA vai manter $\mathrm{g}(0)$ positivo para densidades ainda mais baixas. 0 cálculo das energias dos modos coletivos indica que para $x_{s} \geq 7$ vai aparecer um mínimo em uma posição $q \neq 0$, o que não acontece em RPA. Para $x_{s} \geq 7$, a compress $\underline{i}$ bilidade alcança valores negativos, o que parece indicar uma possível instạ bilidade dos sistemas cuja densidade correspond a estes valores. 


\section{INOICE}

APRESENTAÇAOO

CAPITULO 1 INTRODUÇAOO

1.1 Excitações elementares 2

1.1 .1 Excitaçōes de quasi-particulas 4

1.1 .2 Excitaçōes coletivas 5

1.2 Resposta linear e susceptibilidade 6

1.3 Fator de estrutura e função de correlação dos pares 10 * 4 Regra de soma para a compressibilidade 16

Bibliografia $\quad 19$

CAPITULO 2 METODO AUTOCONSISTENTE NA TEORIA DO GAS DE ELETRONS 20

2.1 Introdução 20

2.2 Método do campo autoconsistente (SCFA) 22

2.2.1 Aproximação semi-clássica 22

2.2.2 Caso quāntico 28

2.3 Resultados para três dimensões 33

2.4 Modificações na SCFA 34

Bibligrafia $\quad 37$ 
CAPITULO 3 APLICAÇAO DE SCFA A UM SISTEMA DE BOSONS 38

3.1 Mëtodo autoconsistente 38

3.2 Gás de bosons tipo esfera dura 43

3.2.1 Espectro de energia 46

3.2.2 Fator de estrutura $e$ função de correlação dos pares 46

3.2 .3 Velocidade do som 47

3.2.4 Massa efetiva 48

3.2.5 Comparação com ${ }^{4} \mathrm{He} \quad 49$

3.3 Gás de bosons: esfera dura e potencial atrativo 51

3.3.1 Fator de estrutura e funçāo de correlação 57

3.3.2 Espectro de energia e velocidade do som 57

3.3.3 Massa efetiva 58

3.3.4 Comparaçāo com ${ }^{4} \mathrm{He} \quad 59$

Bibliografia 61

CAPITULO 4 APLICAÇAO DE SCFA A UM SISTEMA DE FERMIONS 63

4.1 Método autoconsistente 63

4.2 Gäs de Fermi tridimensional 67

4.3 Gás de Fermi unidimensional $\quad 70$

4.4 Resultados $\quad 74$

4.4.1 Cálculo do parâmetro A $\quad 75$

4.4.2 Espectros de energia $\quad 76$

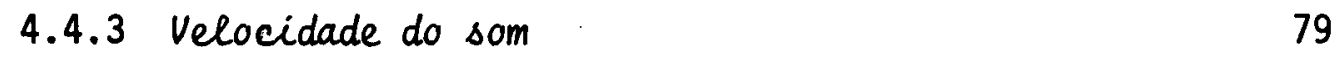

4.4.4 Fator de estrutura e função de correlação $\quad 80$

4.5 Comparação com outros sistemas unidimensionais 80

Bibliografia 83 
CAPITULO 5 GAS DE ELETRONS UNIDIMENSTONAL 84

5.1 Método autoconsistente 85

5.2 Limite para grandes comprimentos de onda 90

5.2.1 Relação de dispersão de plasmon 90

5.2.2 Compressibilidade 91

5.3 Resultados 92

5.3.1 Fator de estrutura e função de correlação dos pares 93

5.3.2 Espectro de energia e compressibilidade 94

Bibliografia 96

APENDICE A CALCULO DA SUSCEPTIBILIDADE DE UM SISTEMA DE PARTICCULAS LIVRES

A.1 Sistema de férmions 97.

A.1.1 Gás de férmions em uma dimensão $\quad 100$

A.1.2 Gás de férmions tridimensional $\quad 101$

A.2 Sistema de basons 103

FIGURAS 


\section{CAPITULO I}

\section{Introdução}

0 objetivo da teoria de muitos corpos é estudar as propriedades de sistemas macroscópicos, baseando-se no conhecimento das forças entre as par tículas, isto é, estudando a maneira pela qual a interação entre as partículas em um sistema denso altera seu comportamento. Uma descrição precisa e completa destes sistemas exige a inclusão do potencial entre as partículas, na equação de Schrobdinger. Em princípio, o conjunto de funções de onda de um sistema de muitos corpos contém toda a informação necessäria; entretanto, a solução exata e direta da equação de Schrßdinger, correspondente ao problę ma, nesse caso, é, em geral, impraticável. É necessārio então desenvolverse hipóteses simplificatórias, com o objetivo de poder resolver o problema. O tipo de aproximação utilizada depende do processo específico a ser estudado e das propriedades nas quais estamos interessados. Assim é criado um mo delo que será adequado para um certo tipo de interação e para um conjunto de densidades do sistema. Então é importante termos conhecimento do trabalho experimental que vem sendo desenvolvido no sistema que nos interessa, e de seus resultados, que terão papel importante no desenvolvimento das hipóteses nas quais baseamos o modelo.

Em estado sólido sabemos quais são as partículas envolvidas e, com boa aproximação, quais as forças que agem entre elas. A dificuldade é que temos cerca de $10^{23}$ partículas por $\mathrm{cm}^{3} \mathrm{e}$, portanto, precisamos resolver um proble ma de muitos corpos. A procura e a utilização de modelos aproximados para estudar sistemas reais de um ponto de vista determinado, tão precisamente 
quanto possível, é um desafio e uma atração da física do estado sólido.

0 trabalho por nós desenvolvido constitui-se na aplicação de uma técnica de aproximação ao estudo de sistemas compostos por bosons e fermions, à temperatura de $0^{\circ} \mathrm{K}$. Vamos então estudar líquidos quânticos, isto é, sistemas homogêneos compostos por partículas que interagem fortemente entre si,em temperacuras suficientemente baixas, de modo que os efeitos quânticos representem papel importante. Os sistemas de ${ }^{3}$ He e ${ }^{4}$ He líquidos são os melho res exemplos reais de sistemas quânticos, como mostram os trabalhos experimentais a baixas temperaturas. Os aspectos do comportamento dos eletrons de condução em metais que não são grandemente afetados pela natureza periōdica do potencial iônico, tambēm podem ser estudados considerando-se o gás de ele trons como um líquido quântico.

\section{1 - EXCITAÇÕES ELEMENTARES}

O objetivo de um trabalho teórico dedicado ao estudo de propriedades de um sistema em geral, não é apenas tentar estimar o estado fundamental do sistema, mas também calcular as energias dos estados excitados que estão ligados com o estado fundamental. Os trabalhos experimentais estão associados à resposta do sistema a um estímulo externo. Tais experiências em geral modificam o estado do sistema para um estado de maior energia. Então,como gran de parte das propriedades do sistema surge dessa mudança de estado de energia, vamos nos interessar principalmente por esses estados excitados. O pro cesso de levar o sistema a um estado de energia mais alta será estudado como um processo onde se supõe que alguma coisa é criada quando nada havia an- 
tes - cria-se uma excitação elementar no sistema.

Para podermos considerar o sistema como um conjunto de excitações elementares que interagem fracamente entre si, elas devem ter uma energia bem definida. Isto acontece ${ }^{(1)}$ quando a excitação pode ser considerada como ten do vida longa. Porém, o sistema que estamos considerando é composto por par tículas fortemente interagentes, e então precisamos examinar se é razoável imaginar que as excitações elementares no sistema tenham longa vida. Seu dẹ caimento pode ocorrer por espalhamento com outras excitações elementares ou com partículas do estado fundamental. o primeiro tipo de decaimento é prati camente desprezível em sistemas a baixas temperaturas, quando temos um nümero relativamente pequeno de excitações elementares. A segunda possibilidade de decaimento apresenta também baixa probabilidade porque, para os sistemas de interesse, existem limitações no espaço de fase disponível para o decai mento de uma excitação de baixo momentum ou grande comprimento de onda. Então, para que as excitações elementares tenham um tempo de vida longo, é necessārio considerarmos sistemas a baixas temperaturas e limitarmos o estudo a fenômenos que envolvem frequências suficientemente baixas e comprimentos de onda comparativamente grandes. Quando isso não acontece podemos ain da descrever um certo fenômeno físico em termos das excitações envolvidas, mas se torna essencial levar em conta o fato de que as excitações possuem um tempo de vida finito.

As excitações elementares de relativamente baixa energia, em um siste ma de muitas partículas, podem ser divididas em duas categorias principais ${ }^{(2)}$ excitações de quasi-particula, que são excitações de partícula única modificadas, e excitações coletivas, que surgem das correlações entre o movimento 
das partículas, devido à interação entre elas.

\section{1 .1 - Excitações de quasi-particulas}

Para entender o conceito de quasi-partícula ${ }^{(3)}$, vamos examinar a seguinte situação: supondo um gás de partículas livres (sem interação), podemos aumentar a energia de uma das partículas sem afetar em nada as demais. Se o sistema estava originalmente no seu estado fundamental, este processo pode ser descrito como criando uma excitação elementar. Se agora aumentarmos a energia de outra partícula, as energias das excitações se somariam para originar a energia do sistema duplamente excitado acima do seu estado fundamental. Estamos assim criando excitações de partícula livre. Se supomos que as partículas do sistema considerado interagem entre si, esperamos que estas excitações de partícula vão decair, jā que agora a partícula excitada sofre espalhamento com as partículas não excitadas e sua energia e momentum seriam gradualmente perdidos. Entretanto, se as partículas obedecem ao princípiode exclusão de Pauli, e, se a energia das excitações for pequena, haverá muito poucos estados vazios para os quais a partícula possa ser espalhada. Espera-se então que a excitação tenha um tempo de vida suficientemente longo para que a descrição em termos de partículas seja adequada. As energias das excitações diferem daquelas de partículas livres, devido à existência de interações. Ao mover-se, a partícula excitada perturba as partículas próximas, afastando umas e arrastando outras. Então, a quasi-partícula pode ser pensa da como uma partícula acompanhada por uma "nuvem" formada pela distorção na sua vizinhança, ocasionada pela existência de interação entre as partīculas. Isto é, a partícula se apresenta como que "vestida" por uma nuvem de auto- 
energia.

\section{1 .2 - Excitações coletivas}

As excitações coletivas relevantes neste trabalho, ou modos coletivos, são semelhantes a ondas de som, por corresponderem a oscilações da densidade das partículas, isto é, ao movimento coerente do sistema como um todo. Entre tanto, diferem de ondas de som quanto ao mecanismo responsável pelas oscilações. Em uma onda de som as colisões entre as partículas agem como uma força restauradora para trazer a densidade de volta ao seu valor médio. Nos modos de excitação coletiva, o campo de força médio criado por um grande número de outras partículas age como força restauradora. As colisões entre as partí culas tendem a modificar esse campo médio, e, consequentemente, atenuar as ex citações. Para que estes modos coletivos possam existir como excitações elementares bem definidas do sistema de mitos corpos, eles devem possuir um tem po de vida suficientemente longo, antes de decaírem em pares de quasi- partículas. Esse decaimento éemgeral energeticamente possível, mas pode ter peque na probabilidade devido a efeitos de coerência entre os elementos de matriz importantes. Exemplos de excitações coletivas em sistemas de muitas partícu las são os fonons, plasmons, excitons, polarons, magnons, rotons, polaritons.

Em um sistema de muitos corpos um aspecto importante é a correlação en tre as posições das partículas, devido às interações mūtuas. Estas correla ções dão origem a importantes efeitos de coerência. Por exemplo, quando o mo do de excitação coletiva existe e pode ser considerado como uma excitação ele 
mentar, em geral ele é o modo de excitação dominante do sistema, embora seja apenas um único modo coletivo, comparado com o contínuo de excitações de quasi-partículas. Isto acontece porque um grande número de partículas toma parte no modo coletivo, e as correlações responsáveis pelo modo coletivo originam também fatores de coerência cujo efeito é reduzir drasticamente a probabi lidade de excitar pares de quasi-partículas.

\section{2 - RESPOSTA LINEAR E SUSCEPTIBILIDADE ${ }^{(4)}$}

Qualquer experimento efetuado em um sistema físico relaciona a excitação do sistema com um estímulo externo, através da medida da resposta ao estî mulo. Se a interação entre a excitação externa e o sistema é suficientemente fraca, a resposta do sistema pode ser considerada linear e é determinada completamente pelas propriedades que o sistema apresenta quando isolado. Consequen temente, experimentos de interação fraca oferecem o método ideal para estudar o comportamento de um sistema de muitas partículas.

Seja $\mathrm{H}_{\mathrm{O}}$ a hamiltoniana que descreve um sistema isolado. Se introduzir mos uma perturbação que vamos representar por $\lambda \phi$, a hamiltoniana do sistema passa a ser escrita como $H_{0}+\lambda \phi$. 0 termo linear em $\lambda$, da diferença entre os valores médios de um observāvel, calculados com e sem a perturbação externa, é a resposta linear ao campo perturbador, do observável considerado.

Vamos examinar o efeito de uma perturbação externa $\phi(\vec{r}, t)$ aplicada a um sistema quântico. Esta perturbação pode ser, por exemplo, um campo de pres sões ou um potencial eletromagnético, no caso de um sistema de partículas carregadas eletricamente. A existência dessa perturbação farā com que apareça u ma flutuação $\rho(\vec{r}, t)$ na densidade do sistema: 


$$
\rho(\vec{n}, t)=\sum_{\bar{q}} \int d \omega \rho(\vec{q}, \omega) e^{i(\vec{q} \cdot \vec{n}-\omega t)} .
$$

Supondo aproximação linear, a resposta $\chi(\vec{q}, \omega)$ à perturbação aplicada serā:

$$
\chi(\vec{q}, \omega)=\frac{\langle\rho(\vec{q}, \omega)\rangle}{\phi(\vec{q}, \omega)},
$$

onde $\phi(\vec{q}, \omega)$ é a transformada de Fourier da perturbação externa e a média é fei ta sobre todos os estados do sistema. $\quad \times(\vec{q}, \omega)$ é então a susceptibilidade ge neralizada do sistema, que representa sua resposta a uma perturbação externa.

A hamiltoniana do sistema quântico composto por $\mathrm{N}$ partículas pode ser escrita da maneira usual

$$
H_{0}=\sum_{i} \frac{p_{i}^{2}}{2 m}+\frac{1}{2} \sum_{\bar{q}} \varphi(\vec{q})\left[\rho_{\vec{q}} \rho_{-\bar{q}}-N\right] \text {, }
$$

onde $\varphi(\vec{q})$ representa a interação entre as partículas e $\rho_{\vec{q}} \bar{e} \circ$ operador densi dade, $\quad \rho \rightarrow \vec{q}=\sum_{i} \exp \left(-i \vec{q} \cdot \vec{n}_{i}\right)$.

Vamos supor que a perturbação seja introduzida no sistema de maneira adiabática, e que ela possa ser considerada como uma perturbação fraca. Supo mos que a interação é ligada em $t=-\infty$, e cresce lentamente até seu valor to tal em $t=0$. A hamiltoniana do sistema fica então:

$$
H=H_{0}+H_{1} \text {, }
$$


8

onde $\mathrm{H}_{1}$ é a hamiltoniana da perturbação

$$
H_{1}=\lim _{\eta \rightarrow 0} \sum_{\bar{q}}\left[\rho_{\bar{q}}^{+} \phi(\tilde{q} \omega) e^{-i \omega t} e^{\eta t}+c . c .\right] .
$$

- A introdução do infinitésimo $n$ garante a condição adiabātica.

A resposta $X(\vec{q}, \omega)$ à perturbação serā calculada na aproximaçãc de resposta linear, supondo que o sistema está inicialmente no seu estado fundamental. A equação de Schrddinger dependente do tempo é:

$$
i \hbar \frac{\partial}{\partial t} \psi(t)=\left(H_{0}+H_{1}\right) \Psi(t) \text {, }
$$

onde $\phi(t) \bar{e}$ a autofunção do sistema total.

Sejam $\psi_{n}$ e $E_{n}$ respectivamente os autoestados e autovalores de $H_{0}$. Pode mos então expandir $\psi(t)$ em termos das $\psi_{n}$, que são funções de onda de sistema de muitos corpos e não sabemos calcular exatamegte,

$$
\Psi(t)=\sum_{n} a_{n}(t) \Psi_{n} e^{-\frac{i}{\hbar} E_{n} t},
$$

onde $a_{n}(t)$ são os coeficientes da expansão que, como o sistema está inicialmente no estado fundamental, devem obedecer às condições de contorno $a_{n}(t=-\infty)=\delta_{n o}$.

Os coeficientes da expansão podem ser calculados usando teoria de per turbação e as Eq̨s. (1.3) a (1.7). Conservando apenas os termos lineares na perturbação, temos: 
9

$$
\begin{aligned}
a_{n}(t) & =\frac{\phi(\bar{q}, \omega)\left(\rho_{\bar{q}}^{+}\right)_{n_{0}} e^{-i\left(\omega-\omega_{n 0}+i \eta\right) t}}{\hbar\left(\omega-w_{n 0}+i \eta\right)}- \\
& -\frac{\phi^{*}(\bar{q}, \omega)\left(\rho_{\bar{q}}\right)_{n_{0}} e^{i\left(\omega+\omega_{n 0}-i \eta\right) t}}{\hbar\left(\omega+\omega_{n 0}-i \eta\right)},
\end{aligned}
$$

onde $\left(\rho_{\vec{q}}\right)_{n o}$ são os elementos de matriz exatos entre as autofunções de $H_{0}$ e $\omega_{n o}$ são as correspondentes frequências de excitação $\hbar \omega_{n o}=E_{n}-E_{0}$.

0 valor esperado de $\rho_{\mathfrak{q}}$ nos autoestados da hamiltoniana completa, conservando apenas termos lineares nos coeficientes $a_{n}(t)$, pode ser escrito como:

$$
\left\langle\rho_{\bar{q}}(t)\right\rangle=\sum_{n}\left[\left(\rho_{\vec{q}}\right) a_{0 n}(t) e^{-i \omega_{n 0} t}+\left(\rho_{\vec{q}}\right)_{n 0} a_{n}^{+}(t) e^{i \omega_{n 0} t}\right]
$$

Este resultado pode ser simplificado consideravelmente se usarmos 0 fato que se $(\rho \vec{q})_{\text {no }} \neq 0$, então $\left(\rho \frac{\hbar}{q}\right)_{\text {no }}=0$; isto $\bar{e}$, o mesmo par de estados não pode ser acoplado simultaneamente por $\rho_{q}$ e $\rho \frac{t}{q}$... Substituindo os valores de coeficientes $a_{n}(t)$ na Eq. (1.9), ficamos com

$$
\begin{aligned}
& \left\langle\rho_{\vec{q}}(t)\right\rangle=\frac{1}{\hbar} \phi(\vec{q}, \omega) e^{-i(\omega+i n) t} \times \\
& \times \sum_{n}\left[\frac{\left|\left(\rho_{q}^{+}\right)_{n 0}\right|^{2}}{\omega-\omega_{n 0}+i \eta}-\frac{\left|\left(\rho_{t}\right)_{n 0}\right|^{2}}{\omega+\omega_{n 0}+i n}\right] .
\end{aligned}
$$

Supondo que o sistema é invariante frente à reflexão temporal e usando

$$
\left\langle\rho_{\bar{q}}(t)\right\rangle=\langle\rho(\bar{q}, \omega)\rangle e^{-i \omega t}
$$


chega-se à expressão para a susceptibilidade generalizada do sistema:

$$
\chi(\vec{q}, \omega)=\frac{1}{\hbar} \sum_{n}\left|\left(\rho_{\bar{q}}^{+}\right)_{n 0}\right|^{2}\left\{\frac{1}{\omega-\omega_{n 0}+i n}-\frac{1}{\omega+\omega_{n 0}+i n}\right\} .
$$

Como $n \rightarrow 0$, podemos escrever:

$$
\operatorname{Re} \chi(\vec{q}, \omega)=\frac{1}{\hbar} P \sum_{n}\left|\left(\rho_{\vec{q}}^{+}\right)_{n 0}\right|^{2}\left(\frac{2 \omega_{n 0}}{\omega^{2}-\omega_{n 0}^{2}}\right)
$$

e

$$
J_{m} X(\vec{q}, \omega)=-\frac{\pi}{\hbar} \sum_{n}\left|\left(\rho_{\vec{q}}\right)_{n 0}\right|^{2}\left[\delta\left(\omega-\omega_{n 0}\right)-\delta\left(\omega+\omega_{n 0}\right)\right],
$$

onde o símbolo $P$ indica que devemos tomar a parte principal da expressão. No apêndice A é obtida em detalhe a expressão para a susceptibilidade de sistemas quânticos sem interação.

\section{3 - FATOR DE ESTRUTURA E'FUnÇÃO DE CORRELAÇAO dOS PARES}

Um exemplo típico de experimento para estudar propriedades de líquidos quânticos neutros é o espalhamento inelástico de um feixe de partículas (neutrons) pelo líquido. Vamos supor que o potencial de interação entre o sistema e a excitação externa depende apenas da posição relativa das partículas do sistema e do feixe incidente:

$$
H_{\text {int }}=\sum_{i} \phi\left(\vec{n}_{i}-\vec{R}\right)
$$


11

Escrevendo em termos dos componentes de Fourier, temos

$$
H_{\text {int }}=\sum_{i} \sum_{\bar{q}} \phi_{\vec{q}} e^{i \vec{q} \cdot\left(\vec{n}_{i}-\vec{R}\right)}=\sum_{\bar{q}} \phi_{\bar{q}} \rho_{\vec{q}}^{+} e^{-i \vec{q} \cdot \vec{R}},
$$

onde $\underset{\vec{q}}{\phi} \underset{q}{\rho}$ são respectivamente as transformadas de Fourier da excitação ex terna e da densidade de partículas $\rho(\vec{r})=\sum_{i} \delta\left(\vec{r}-\vec{r}_{i}\right)$.

Usando teoria de perturbação, podemos calcular a probabilidade $P(\vec{q}, \omega)$ por unidade de tempo de que a partícula transfira momentum $\hbar_{\vec{q}}$ e energia $\hbar_{\omega}$ ao sistema $(5)$. 0 resultado $\bar{e}$

$$
P(\vec{q}, \omega)=2 \pi\left|\phi_{\vec{q}}\right|^{2} \bar{S}(\vec{q}, \omega) \text {, }
$$

onde definimos

$$
\bar{S}(\bar{q}, \omega)=\frac{1}{\hbar} \sum_{n}\left|\left(\rho_{\bar{q}}^{+}\right)_{n 0}\right|^{2} \delta\left(\omega-\omega_{n 0}\right) \text {. }
$$

o fator de estrutura' dinâmico $\bar{S}(\vec{q}, \omega)$ engloba todas as propriedades do sistema de muitas partículas que são relevantes ao espalhamento do feixe de neutrons, e é a māxima informação que se pode obter sobre o comportamento do sistema em tal experimento. Como para $T=0^{\circ} \mathrm{K}$ as frequência $\omega_{\text {no }}$ são todas positivas, $\bar{s}(\vec{q}, \omega)=0$ para $\omega<0$ e $\bar{s}(\vec{q}, \omega)$ e sempre real.

Podemos relacionar o fator de estrutura dinâmico com a parte imaginá ria da susceptibilidade generalizada do sistema par $\vec{q} \neq 0$, usando a Eq. (1.12b)

$$
I_{m} \chi(\vec{q}, \omega)=-\pi[\bar{S}(\vec{q}, \omega)-\bar{S}(\vec{q},-\omega)] \text {, }
$$


e a susceptibilidade completa pode ser obtida de $\bar{S}(q, \omega)$, usando

$$
X(\vec{q}, \omega)=\int_{-\infty}^{\infty} d \omega^{\prime} \bar{S}\left(\vec{q}, \omega^{\prime}\right)\left\{\frac{1}{\omega-\omega^{\prime}+i \eta}-\frac{1}{\omega+\omega^{\prime}+i \eta}\right\} .
$$

Supondo que não se possa analisar a energia das partículas (neutrons) emergentes, o que se mede é a seção de choque diferencial em função de ângulo $\theta$ de espalhamento. Se supomos ainda que o momentum e a energia transferi dos sejam essencialmente independentes, podemos obter para a probabilidade correspondente

$$
P(\theta)=\alpha_{n}\left|\phi_{\vec{q}}\right|^{2} N \vec{S}(\vec{q}),
$$

onde $\alpha_{n}$ depende das partículas do feixe incidente e o fator de estrutura estātico è definido por:

$$
\bar{S}(\vec{q})=\frac{\hbar}{N} \int_{0}^{\infty} d w \bar{S}(\vec{q}, \omega) .
$$

A relação entre o fator de estrutura $\bar{S}(\vec{q})$ e a susceptibilidade $\chi(\vec{q}, \omega)$, quando $\vec{q} \neq 0$, $\bar{e}$

$$
\bar{S}(\vec{q})=-\frac{\hbar}{\pi N} \int_{0}^{\infty} d \omega Y_{m} \chi(\vec{q}, \omega) .
$$

Por outro 1ado, usando as Eqs.(1.15) e (1.18) vamos obter, para o fator de estrutura $\overrightarrow{\mathrm{s}}(\overrightarrow{\mathrm{q}})$ 
13

$$
\bar{S}(\vec{q})=\frac{1}{N} \sum_{n}\left|\left(\rho_{\vec{q}}^{+}\right)_{n o}\right|^{2}=\frac{1}{N}\left\langle 0\left|\rho_{\vec{q}} \rho_{\bar{q}}^{+}\right| 0\right\rangle .
$$

Podemos então separar na expressão do fator de estrutura a contribuição de transferência de momentum nula. Como $\rho_{0}=N$, vamos ficar com

$$
\bar{S}(\vec{q})=\frac{1}{N}\left\{\left\langle 0\left|p_{q} \rho_{\bar{q}}^{+}\right| 0\right\rangle\right\}_{\bar{q} \neq 0}+N \delta_{q_{0}}
$$

Nesta definição para o fator de estrutura,que é a utilizada por Pines ${ }^{(6)}$, temos $\bar{S}(0)=N$. Podemos então definir um "novo" fator de estrutura

$$
S(\vec{q})=\bar{S}(\vec{q})-N \delta_{\vec{q}_{0}}=\frac{1}{N}\left\langle 0\left|\rho_{\vec{q}} \rho_{\vec{q}}^{+}\right| 0\right\rangle-N \delta_{\vec{q} 0}
$$

que é nulo para $\vec{q}=0$.

Com essa nova definição, e o corrępondente $S(\vec{q}, \omega)$, as relações entre a susceptibilidade generalizada e o fator de estrutura valem para todo $\vec{q}$. Nes te trabalho, vamos usar a definição (1.22).

o fator de estrutura permite tambēm obter-se informação detalhada com relação a correlações espaciais em um sistema de muitas partículas. Vamos con siderar a evolução temporal de uma flutuação de densidade $\rho_{\vec{q}}^{t}$. Se H é a hamiltoniana total do sistema, temos, na representação de Heisenberg ${ }^{(7)}$ :

$$
\rho_{\vec{q}}^{+}(t)|0\rangle=e^{\frac{i}{t} H t} \rho_{\vec{q}}^{+} e^{-\frac{i}{\hbar} H t}|0\rangle \text {. }
$$


14

A correlação dinâmica entre uma flutuação de densidade em $t=0$ outra no tempo t é expressa pela função de correlação da densidade,

$$
p(\vec{r}, t)=\sum_{\bar{q}} p(\vec{q}, t) e^{i \vec{q} \cdot \vec{r}},
$$

com

$$
p(\vec{q}, t)=\frac{1}{N}\left\langle 0\left|\rho_{\bar{q}}(t) \rho_{\bar{q}}^{+}(0)\right| 0\right\rangle .
$$

Usando a Eq.(1.20) e introduzindo o conjunto completo de autoestados de H, obtém-se

$$
\begin{aligned}
& p(\vec{q}, t)=\frac{1}{N} \sum_{n}\left\langle 0\left|e^{i_{n} H t} \rho_{\vec{q}} e^{-i}\right| n\right\rangle\left\langle n\left|\rho_{\hat{q}}^{+}\right| 0\right\rangle= \\
& =\frac{1}{N} \sum_{n}\left|\left(\rho_{\bar{q}}^{t}\right)_{n p}\right|^{2} e^{-i \omega_{n 0} t},
\end{aligned}
$$

e assim

$$
p(\vec{n}, t)=\frac{1}{N} \sum_{n} \sum_{\vec{q}}\left|\left(\rho_{\bar{q}}^{t}\right)_{n 0}\right|^{2} e^{i\left(\vec{q}, \vec{n}-\omega_{n 0} t\right)}
$$

A função de correlação estātica da densidade pode ser escrita como 
15

$$
p(\vec{r})=p(\vec{r}, 0)=\frac{1}{N} \sum_{\vec{q}}\left\langle 0\left|\rho_{q} \rho_{\vec{q}}^{+}\right| 0\right\rangle e^{i \vec{q} \cdot \vec{r}}
$$

on

$$
p(\vec{n})=\sum_{q} S(\vec{q}) e^{i \vec{q} \cdot \vec{n}}+N
$$

onde usamos a Eq. (1.22).

Por outro lado, a função de correlação estática da densidade está relą cionada com a função de correlação dos pares $g\left(F^{\prime}\right)$. Supondo um sistema de par tículas puntuais

$$
\begin{aligned}
p(\vec{r}) & =\frac{1}{N} \int\left\langle 0\left|\rho\left(\vec{r}+\vec{r}^{\prime}\right) \rho\left(\vec{r}^{\prime}\right)\right| 0\right\rangle d \vec{r}^{\prime}= \\
& =\frac{1}{N} \int\left\langle 0\left|\sum_{i, j} \delta\left(\vec{r}+\vec{r}^{\prime}-\vec{r}_{i}\right) \delta\left(\vec{r}^{\prime}-\vec{r}_{j}\right)\right| 0\right\rangle d \vec{r}^{\prime}
\end{aligned}
$$

Para um sistema com invariança translacional, como as coordenadas das partícu las $\underline{i}$ e $\underline{j}$ comutam, já que são observadas simultaneamente, temos

$$
\begin{aligned}
p(\vec{n}) & =\frac{1}{N}\left\langle 0\left|\sum_{i, j} \delta\left(\vec{n}+\vec{n}_{j}-\vec{n}_{i}\right)\right| 0\right\rangle= \\
& =\delta(\vec{n})+(N-1) \quad g(\vec{n}),
\end{aligned}
$$

onde a função de correlação dos pares é expressa por 


$$
g(\vec{n})=\frac{1}{N(N-1)}\left\langle 0\left|\sum_{i \neq j} \delta\left(\vec{r}+\vec{r}_{j}-\vec{r}_{i}\right)\right| 0\right\rangle .
$$

Na Eq.(1.28), a primeira parcela do lado direito representa as correla ções de cada partícula com si mesma, enquanto que a segunda parcela representa as correlações entre pares de partículas. Então, a função $g(\vec{r})$ expressa a probabilidade de que se uma partícula é observada em uma certa posição $\vec{r}_{0}$, outra partícula será encontrada em $\vec{r}_{0}+\vec{r}_{\text {. }}$ Usando as Eqs.(1.26) e (1.28), po demos mostrar que, para um sistema onde o número de partículas é muito grande, o fator de estrutura e a função de correlação dos pares $g(\vec{r})$ estão relacionados através da integral de Fourier, isto é

$$
\frac{1}{N}[S(\vec{q})-1]=T . F \cdot[g(\vec{n})-1] \text {. }
$$

\section{4 - REGRA DE SOMA PARA A COMPRESSIBILTDADE}

No limite de grandes comprimentos de onda, a susceptibilidade estática $x(\vec{q}, 0)$ representa a resposta da densidade $\rho$ do sistema a um campo de força es tático, que varia lentamente no espaço. Então, deve estar relacionada com a compressibilidade $K$ do sistema. A relação entre elas é facilmente estabelecí da $(8,9)$, considerando a descrição macroscópica da resposta do sistema ao potencial escalar $\phi(\vec{r})$. A força sentida por uma partícula devido ao potencial estático é: 


$$
\vec{F}(\vec{r})=-i \vec{q} \phi(\vec{q}) e^{i \vec{q} \cdot \vec{r}}+c . c .
$$

Se supomos que essa força longitudinal varia lentamente no espaço, podemos de finir a força média por unidade de volume do sistema como $\rho \vec{F}(\vec{r}) \cong N \vec{F}(\vec{r})$, considerando apenas termos lineares no potencial. O efeito deste campo de forças é deformar o sistema, criando uma flutuação de densidade $\delta \rho(\vec{r})$, que, por sua vez, origina uma distribuição adicional de pressões

$$
\delta P(\vec{n})=\frac{1}{N K} \delta \rho(\vec{n})
$$

onde $K \bar{e}$ a compressibilidade macroscópica.

A distribuição $\delta P(\vec{r})$ deve ser tal que a força externa seja contrabalan çada pelo termo grad SP. Ou seja, a distribuição de pressões vai contrabalançar o efeito do potencial externo

$$
\delta P(\vec{n})=-N \phi(\vec{n})=-N \phi(\vec{q}) e^{i \vec{q} \cdot \vec{n}}+c \cdot c .
$$

e

$$
\delta \rho(\vec{n})=-k N^{2} \phi(\vec{q}) e^{i \vec{q} \cdot \vec{n}}+c \cdot c .
$$

ou ainda

$$
\delta \rho(\bar{q})=-K N^{2} \phi(\bar{q}) .
$$


Porém, $\delta \rho(\vec{q})$ é a flutuação de densidade induzida pelo potencial externo $\phi(\vec{q})$, para $\vec{q} \rightarrow 0$. Então, lembrando a definição de susceptibilidade genera lizada, Eq.(1.2), podemos escrever

$$
\lim _{\vec{q} \rightarrow 0} \chi(\vec{q}, 0)=-K N^{2}=-\frac{N}{m s^{2}}
$$

onde usamos a relação usual entre a compressibilidade e a velocidade do som ${ }^{(10)}$. 


\section{BIBLIOGRAFIA}

\section{CAPÍTULO 1}

1. XD. Pines "Elementary excitations in solids" (W.A. Benjamin, Inc., New York, 1964) capitulo 1.

2. KD. Pines "The many-body problem" (W.A. Benjamin, Inc., New York, 1962) capítulo 1 .

3. P.L. Taylor "A quantum approach to the solid state" (Prentice-Hall, En glewood Cliffs, NJ, 1970) capítulo 1.

4. P. Nozières \& D. Pines Il Nuovo Cimento (X) 9 , 470 (1958) Referência (3), pāg. 123.

5. D. Pines \& P. Nozières "The theory of quantum liquids" (W.A. Benjamin, Inc., New York, 1966) pāg. 85.

6. Veja, por exemplo, refs. (1), (2) e (5).

7. Veja. referência (1), pāg. 130

8. D. Pines em "Lectures on the many-body problem", vol, 2, E.P.Caianello, Ed. (Academic Press, New York, 1964) p. 15.

9. E. Feenberg "Theory of quantum fluids" (Academic Press, New York,1969)

10. Veja, por exemplo, C. Kittel "Quantum theory of solids" (J. Wiley, New York, 1963). 


\section{CAPİTULO 2}

Mètodo autoconsistente na teoria do gās de eletrons

\section{1 - INTRODUÇAO}

Correlações no gás de elétrons podem ser expressas em termos da cons tante dielētrica. A constante dielétrica $\varepsilon(\vec{q}, w)$, dependente da frequência e vetor de onda, descreve a resposta de um sistema a um campo elétrico aplicado

e é uma importante grandeza no estudo de muitas propriedades físicas do siste ma. 0 gās de eletrons é estudado em um fundo positivo uniforme neutralizan te. As propriedades metālicas que dependem fortemente das interações ele tron-eletron também podem ser estudadas por meio da constante dielétrica. Pa ra estender esse modelo a metais, é necessārio supor que a constante dielétrị ca não é alterada pela natureza discreta dos ions.

A constante dielétrica tem sido calculada em värias aproximações. A aproximação RPA (Random Phase Approximation) ${ }^{(1)}$ consiste em desprezar em uma soma de exponenciais com diferentes fases, os termos de fases aleatórias, quando comparados com $N$, o número total de partículas. Resulta então no tra tamento da parte de longo alcance do potencial Coulombiano como um campo ex terno polarizante.

0 método RPA dá boa descrição para os modos de excitação de plasmons, e fenômenos de blindagem a grande comprimento de onda. Porém sua validade é limitada à região de altas densidades $\left(r_{s} \ll 1\right.$, onde $r_{s}=r_{0} / a_{0}, r_{0} \bar{e}$ a distân cia média entre as partỉculas e a $a_{0}$ o raio de Bohr). Na região de densida des metālicas, $1.5<\mathrm{r}_{\mathrm{s}}<6$, RPA prevê uma função distribuição dos pares que é 
bastante negativa para pequenas separações entre as partículas. Isto aconte ce porque nesta aproximação não são distinguidos os efeitos de correlação de spins paralelos e antiparalelos. A aproximação RPA não inclui efeitos de in tercâmbio que influem na parte de spin paralelo, is to é, para grandes transfe rências de momentum, este termo vai cancelar metade da interação direta: fisi camente se espera que eletrons de spin paralelos não sintam a parte de curto alcance da interação, jā que o princỉpio de Pauli faz com que eles se mante nham afastados. Apenas os eletrons de spin antiparalelos interagem pela parte de grande transferência de momentum da interação coulombiana. Assim, a aproximação RPA falha quando $\vec{q}>\vec{q}_{F}$, vetor de onda de Fermi, em densidades metālicas. No limite de altas densidades, as contribuições importantes para a energia de correlação surgem das baixas transferências de momentum e esta aproximação apresenta resultados satisfatórios.

Hubbard (2) e Noziēres \& Pines (3) propuseram modificação na RPA, basea das essencialmente na mesma idéia física: para grandes transferências de mo mentum (baixas densidades) a principal contribuição à energia de correlação surge das interações de eletrons de spin antiparalelos.

Hubbard levou em conta os termos de intercâmbio através de uma soma quase infinita de gräficos na energia de correlação, enquanto que Nozières \& Pines calcularam a energia de correlação por meio de uma interpolação entre as contribuições de grandes e pequenas transferências de momentum. A corre ção de Hubbard originou uma nova expressão para a constante dielétrica, que melhorou bastante a energia de correlação do gás de eletrons em densidades me tá1icas. Entretanto, a função distribuição dos pares $g(\vec{r})$ permanece bastan te negativa para pequenos valores de $\vec{r}$, em toda essa região de densidades. 
Esse fato indica falha, nesta aproximação, em levar em conta as interações de curto alcance: a constante dielétrica de Hubbard inclui correções de campo lo cal apenas para levar em conta o "exchange hole" na aproximação de HartreeFock. Entretanto, na região onde os valores da densidade são tais que $1.5<\mathrm{r}_{s}<6$, as interaçoes elétron-elétron tem papel importante, e então de vem também ser consideradas as correções de campo local associadas com o "Cou lomb hole".

\section{2 - METODO DO CAMPO ALTOCONSISTENTE}

Singwi et al ${ }^{(4,5)}$ desenvolveram um método autoconsistente (SCFA) para o cálculo da constante dielétrica e do fator de estrutura para metais alcali nos $\left(1<r_{s}<6\right)$, que leva em conta as correlações de curto alcance devidas ao potencial de Coulomb e "exchange". Lste método permite expressar a função dielétrica como um funcional da transformada de Fourier da função correlação dos pares no gās de eletrons.

\section{2 .1 - Aproximação Semi-clássica}

No caso semi-clāssico, a hipótese básica consiste em substituir a fun ção distribuição clássica para duas partículas, $f^{(2)}\left(\vec{r}, \vec{p} ; \vec{r}^{\prime}, \vec{p}^{\prime} \mid t\right)$ por um produto de funções distribuições de uma partícula, introduzindo-se nesse desa coplamento a função de correlação estática de equilíbrio dos pares, $g^{\circ}\left(\vec{r}-\vec{r}^{\prime}\right)$, que expressa a probabilidade combinada de termos de uma partícula em $\vec{r}$ e ou tra em $\vec{r}^{\prime}$.

Escreve-se então

$$
f^{(2)}\left(\vec{r}, \vec{p} ; \vec{r}^{\prime}, \vec{p}^{\prime} \mid t\right)=g^{0}\left(\vec{r}-\vec{r}^{\prime}\right) f^{(1)}(\vec{r}, \vec{p} \mid t) f^{(1)}\left(\vec{r}^{\prime}, \vec{p}^{\prime} \mid t\right) .
$$


A justificativa da introdução da função correlação estática na expres são da função distribuição de duas partículas será feita a posteriori, atra vés dos resultados obtidos. A expressão mais simples que se poderia supor para $f^{(2)}\left(\vec{r}, \vec{p} ; \vec{r}^{\prime}, \vec{p}^{\prime} \mid t\right)$ seria apenas o produto das funções distribuição de cada partícula - o que corresponderia à aproximação RPA.

Com a hipótese feita por Singwi et al, que leva em conta as correla ções de curto alcance, pode-se obter um conjunto fechado de equações de forma autoconsistente.

A transformada de Fourier da função correlação estática de equilỉbrio dos pares, $g^{\circ}(\vec{r})$, é relacionada com o fator de estrutura $S(\vec{q})$ da seguinte for ma :

$$
\text { T.F. }\left[q^{0}(\vec{n})-1\right]=\frac{1}{\rho}[S(\vec{q})-1] \text {, }
$$

ou

$$
g(\vec{n})=1+\frac{1}{\rho} \int \frac{d \vec{q}}{(2 \pi)^{3}} e^{i \vec{q} \cdot \vec{n}}[S(\vec{q})-1] \text {, }
$$

onde $\rho$ é a densidade numérica de partículas.

Por sua vez o fator de estrutura está relacionado com a susceptibilida de do fluido $x(\vec{q}, \omega)$ através da expressão exata ${ }^{(6)}$ :

$$
S(\vec{q})=-\frac{\hbar}{\pi \rho} \int_{0}^{\infty} d \omega \operatorname{Im} \chi(\vec{q}, \omega) .
$$

Para o gás de eletrons a constante dielétrica $\varepsilon(\vec{q}, \omega)$ é dada por 


$$
\frac{1}{E(\vec{q}, \omega)}=1+\phi(\vec{q}) \chi(\vec{q}, \omega),
$$

onde $\phi(\vec{q})=4 \pi e^{2} / q^{2} \bar{e}$ a transformada de Fourier do potencial coulombiano.

Então

$$
S(\vec{q})=-\frac{\hbar}{\pi \rho \phi(\vec{q})} \int_{0}^{\infty} d \omega \quad I_{m}[\epsilon(\vec{q}, \omega)]^{-1} .
$$

Precisamos agora apenas de uma expressão para $\chi(\vec{q}, \omega)$ (ou para a cons tante dielétrica) em função do tafor de estrutura $S(\vec{q})$, para completarmos 0 esquema de equações autoconsistentes.

A hamiltoniana clássica de $\mathrm{N}$ elétrons interagindo entre si, e sob a ação de um campo externo é:

$$
H_{0}=\sum_{i=1}^{N} H_{i}
$$

com

$$
f_{i}=\frac{p_{i}^{2}}{2 m}+\phi_{\text {ext }}\left(\vec{r}_{i}, t\right)+\frac{1}{2} \sum_{j \neq i} \phi\left(\vec{r}_{i}-\vec{r}_{j}\right),
$$

onde $\phi_{\text {ext }}\left(\vec{r}_{i}, t\right) \bar{e}$ a energia potencial da partỉcula $\underline{i}$ na presença do campo ex terno, e $\phi\left(\vec{r}_{i}-\vec{r}_{j}\right) \bar{e}$ a interação dos pares de partículas. No caso de gás de elétrons, $\phi\left(\vec{r}_{i}-\vec{r}_{j}\right) \bar{e}$ a interação coulombiana. Entretanto, o desenvolvimento pode ser feito para uma interação genérica. 
25

A equação de Liouville não relativística, para o caso de $\mathrm{N}$ partículas, pode ser escrita como

$$
\frac{\partial f^{(N)}}{\partial t}+\left[\sum_{i=1}^{N} Q\left(\bar{r}_{i}, \vec{p}_{i}\right)\right] f^{(N)}=0
$$

onde o operador $Q\left(\vec{r}_{i}, \vec{p}_{i}\right) \bar{e}$ expresso por

$$
Q\left(\vec{n}_{i}, \vec{p}_{i}\right)=\vec{v}_{i} \cdot \nabla_{\vec{n}_{i}}-\nabla_{\vec{n}_{i}} \phi_{\text {ext }}\left(\vec{n}_{i}, t\right) \cdot \nabla_{\vec{p}_{i}}+\frac{1}{2} \sum_{i \neq j} \nabla_{\vec{n}_{i}} \phi\left(\vec{n}_{i}-\vec{n}_{j}\right) \cdot \nabla_{\overrightarrow{r_{i}}},
$$

e

$$
f^{(N)} \equiv f^{(N)}\left(\vec{r}_{1}, \vec{p}_{1} ; \bar{r}_{2}, \bar{p}_{2} ; \ldots \bar{r}_{N}, \bar{p}_{N} \mid t\right)
$$

é a função distribuição combinada de $\mathbf{N}$ partîculas.

Integrando a equação de Liouville nas coordenadas e momenta de N-1 par tỉculas, chega-se à equação de movimento para a função distribuição clássica de uma partícula, na presença de um campo externo:

$$
\begin{aligned}
& \frac{\partial f^{(1)}}{\partial t}+\vec{v} \cdot \nabla_{\vec{n}} f^{(1)}-\nabla_{\vec{n}} \phi_{\text {ext }}(\vec{r}, t) \cdot \nabla_{\vec{p}} f^{(1)}- \\
& -\int d \vec{n}^{\prime} d \vec{p}^{\prime} \nabla_{\vec{n}} \phi\left(\vec{n}-\vec{n}^{\prime}\right) \cdot \nabla_{\vec{p}} f^{(2)}=0 \\
& \text { onde } f^{(1)} \equiv f^{(1)}(\vec{r}, \vec{p} \mid t) \text { e } f^{(2)} \equiv f^{(2)}\left(\vec{r}, \vec{p} ; \vec{r}^{\prime}, \vec{p}^{\prime} \mid t\right) \text {. }
\end{aligned}
$$

Assim, a equação de movimeñto da função distribuição de uma partícula envolve a função distribuição de duas partículas. Por sua vez, a equação de 
26

movimento para $f^{(2)}\left(\vec{r}, \vec{p} ; \vec{r}^{\prime}, \vec{p}^{\prime} \mid t\right)$ depende de $f^{(3)}\left(\vec{r}, \vec{p} ; \vec{r}^{\prime}, \vec{p}^{\prime} \mid t\right)$, e assim sucessivamente. Para resolver a Eq.(2.8) é preciso então quebrar essa se quência infinita de equações. Singwi et al propõem que isto seja feito atra vës do desacoplamento expresso na Eq.(2.1), levando em conta, assim, de manei ra aproximada, as correlações de curto alcance entre as partículas, através da função de correlação $g\left(\vec{r}-\vec{r}^{\prime}\right)$.

A função distribuição $f^{(1)}(\vec{r}, \vec{p} \mid t)$ pode ser escrita como a soma de um termo de equilibrio $f_{0}(\vec{p})$ e um termo de correção $f_{1}(\vec{r}, \vec{p} \mid t)$, que exprime 0 afastamento do equilỉbrio devido ao potencial externo (fraco):

$$
f^{(1)}(\vec{r}, \vec{p} \mid t)=f_{0}(\vec{p})+f_{1}(\vec{r}, \vec{p} \mid t)
$$

Usando as Eqs.(2.1) e (2.9), e conservando apenas os termos de primei ra ordem no potencial externo, a Eq.(2.8) pode ser escrita como

$$
\left[\frac{\partial}{\partial t}+\vec{v} \cdot \nabla_{\vec{r}}\right] f_{1}(\vec{n}, \vec{p} \mid t)-\nabla_{\vec{r}} \phi_{e f}(\vec{r}, t) \cdot \nabla_{\vec{p}} f_{0}(\vec{p})=0 \text {, }
$$

com

$$
\phi_{\text {ef }}(\vec{r}, t) \equiv \phi_{\text {ext }}(\vec{r}, t)+\int d \vec{r} \cdot n(\vec{r} ; t) \Psi\left(\vec{r}-\vec{r}^{\prime}\right),
$$

onde

$$
\nabla_{\vec{n}} \psi\left(\vec{r}-\vec{r}^{\prime}\right)=g\left(\vec{r}-\vec{r}^{\prime}\right) \nabla_{\vec{r}} \phi\left(\vec{r}-\vec{r}^{\prime}\right),
$$

e $n(\vec{r}, t)=\int \overrightarrow{d p} f_{1}(\vec{r}, \vec{p} \mid t)=$ densidade de particulas em $(\vec{r}, t)$. 
27

O campo efetivo que a partícula sente pode ser escrito como:

$$
\begin{gathered}
\vec{E}_{e f}(\vec{r}, t)=-\nabla_{\vec{r}} \phi_{e f}(\vec{r}, t)=-\nabla_{\vec{r}} \phi_{\text {ext }}(\vec{r}, t)-\int d \vec{r}^{\prime} \nabla_{r} \phi\left(\vec{r}-\vec{r}^{\prime}\right) n\left(\vec{r}^{\prime}, t\right)- \\
-\int d \vec{r}^{\prime}\left[g\left(\vec{r}-\vec{r}^{\prime}\right)-1\right] \nabla_{\vec{r}} \phi\left(\vec{r}-\vec{r}^{\prime}\right) n\left(r^{\prime}, t\right) .
\end{gathered}
$$

Desta forma aparecem separados no campo efetivo os termos RPA e a cor reção devida à SCFA. Em RPA o campo efetivo é dado pelo campo macroscópico usual, ou seja, o campo externo e o termo de interação entre as partículas, correspondendo às duas primeiras parcelas da soma, sem levar em conta as cor relações. Em SCFA, além do campo externo, ao calcular o campo efetivo leva mos em conta parte da correlação entre as partículas, o que significa conside rar a ültima parcela, além dos termos RPA.

Como a equação de movimento obtida, Eq.(2.10) é linear, sua solução po de ser encontrada considerando-se apenas uma componente de Fourier para o po tencial externo:

$$
\phi_{\text {ext }}(\vec{r}, t)=\phi_{\text {ext }}(\vec{q}, \omega) e^{i(\vec{q} \cdot \vec{r}-\omega t)}+c \cdot c .
$$

Calculando da maneira usual a densidade de carga induzida ${ }^{(7)}$, chega-se à expressão para a susceptibilidade:

$$
\chi(\vec{q}, \stackrel{\omega}{\omega})=\frac{\chi^{0}\left(\vec{q}, w^{*}\right)}{1-\psi(q) X^{0}\left(\vec{q}, \omega^{*}\right)},
$$

onde $\chi^{0}\left(\vec{q}, \omega^{*}\right)$ é a susceptibilidade do mesmo gás sem interação e $\omega^{*}=\omega+$ in, a frequência complexa. 
Para um gás de elétrons, onde o potencial de interação é coulombiano, is to $\vec{e}, \phi(\vec{q})=4 \pi e^{2} / q^{2}$, podemos obter para o potencial efetivo $\psi(\vec{q})$, a partir da Eq. $(2.12)$

$$
\psi(\vec{q})=\phi(\vec{q})[1-G(\vec{q})],
$$

com

$$
G(\vec{q})=-\frac{1}{\rho} \int \frac{d \vec{q}^{\prime}}{(2 \pi)^{3}} \frac{\vec{q} \cdot \vec{q}^{\prime}}{\left(q^{\prime}\right)^{2}}\left[S\left(\vec{q}-\vec{q}^{\prime}\right)-1\right] .
$$

A expressão para a constante dielétrica fica então

$$
\epsilon\left(\vec{q}, \omega^{*}\right)=1-\phi(\vec{q}) \frac{\chi^{0}\left(\vec{q}, \omega^{*}\right)}{1+\phi(\vec{q}) G(\vec{q}) \chi^{*}\left(\vec{q}, \omega^{*}\right)} .
$$

Chegamos assim, no caso de um gás de elétrons, a um conjunto fechado composto pelas Eqs.(2.6), (2.16) e (2.17). Es tas equações deverão ser resol vidas de maneira autoconsistente, permitindo obter-se o fator de estrutura $S(\vec{q})$ para o sistema considerado. A função correlação dos pares $g(\vec{r})$ serä ob tida usando-se na Eq.(2.3) o fator de estrutura autoconsistente.

\section{2 .2 - Caso Quântico}

As equações a serem resolvidas no caso quântico são obtidas dos resul tados clássicos, fazendo-se a substituição do fator de estrutura e da suscep tibilidade do gās sem interação clássicos, por seus análogos quânticos. Es ta hipótese é feita da mesma forma autoconsistente, isto $\bar{e}$, o sistema respon 
de ao potencial blindado através da susceptibilidade de particula livre. Nes se caso, ao levar em conta as correlações de curto alcance, estamos conside rando uma diminuição da densidade eletrônica ao redor de cada partícula, as sociada à repulsão coulombiana (Coulomb hole) e à repulsão de "exchange" (ex change hole), o que não é levado em conta em RPA. Novamente, se desprezar mos as correções de campo local, obtemos as expressões de RPA, enquanto que, se usarmos a expressão do fator de estrutura de Hartree-Fock para calcular o potencial efetivo de interação, chega-se à constante dielétrica de Hubbard.

A hamiltoniana de um sistema de $\mathrm{N}$ partículas de massa me é expressa por

$$
H=\sum_{i=1}^{N} \frac{p_{i}^{2}}{2 m}+\frac{1}{2} \sum_{i \neq j} \phi\left(\vec{r}_{i}-\vec{r}_{j}\right),
$$

que pode ser escrita ${ }^{(8)}$ como

$$
H=\sum_{i=1}^{N} \frac{p_{i}^{2}}{2 m}+\frac{1}{2} \sum_{\vec{q} \neq 0} \phi(\vec{q})\left[\rho_{\vec{q}}^{+} \rho_{\vec{q}}-N\right],
$$

onde a matriz densidade $\rho_{q} \rightarrow \bar{e}$ dada por:

$$
\rho_{\bar{q}}=\sum_{i=1}^{N} e^{-i \vec{q} \cdot \vec{n}_{i}}
$$

Na parcela correspondente à energia potencial na Eq.(2.18), o fator $N$ deve ser subtraído porque corresponde ao termo $i=j$ no potencial de interação no espaço real, $\phi\left(\vec{r}_{i}-\vec{r}_{j}\right)$. A soma não inclui o termo $\vec{q}=0$, que é cancelado pe lo fundo positivo.

A equação de movimento da matriz densidade ${ }^{(9)}$, expressa no caso geral 
30

por

$$
\ddot{\rho}_{\vec{q}}=\frac{1}{(i \hbar)^{2}}\left[\left[\rho_{\vec{q}}, H\right], H\right] \text {, }
$$

que pode ser escrita como

$$
\ddot{\rho}_{\vec{q}}=-\sum_{i=1}^{N}\left(\frac{\vec{q} \cdot \vec{p}_{i}}{m}+\frac{\hbar q^{2}}{2 m}\right)^{2} e^{-i \vec{q} \cdot \vec{r}_{i}}-\sum_{\vec{q}^{\prime}} \phi\left(\vec{q}^{\prime}\right) \frac{\vec{q} \cdot \vec{q}^{\prime}}{m} \rho_{\vec{q}^{-}-\vec{q}^{\prime}} \rho_{\vec{q}^{\prime}},^{(2.20)}
$$

onde o primeiro somatório representa a contribuição da energia cinética e o segundo surge das interações entre as partículas.

No segundo somatōrio, o termo com $\vec{q}^{\prime}=\vec{q}$ corresponde ao termo de inte ração que aparece em RPA:

$$
-\frac{q^{2}}{m} \phi(\vec{q}) \rho_{0} \rho_{\vec{q}}=-\omega_{p}^{2} \rho_{\vec{q}},
$$

onde $\omega_{p} \bar{e}$ a frequência generalizada de plasma, expressa por $\omega_{p}^{2}=\mathrm{Nq}^{2} \phi(\mathrm{q}) / \mathrm{m}$. Para um sistema coulombiano, obtém-se para a frequência de plasma a expressão usual $\omega_{\mathrm{p}}^{2}=4 \pi \mathrm{Ne}^{2} / \mathrm{m}$.

A soma, abrangendo os termos $\vec{q}^{\prime} \neq \vec{q}$, que não $\bar{e}$ considerada em RPA, cor responde a uma correção pequena, porque apresenta pordutos de flutuações de densidade $\rho_{q}$, cada uma delas expressa por soma de fases aleatórias, quando $\overrightarrow{\mathrm{q}}^{\prime} \neq 0$.

$$
\begin{aligned}
\ddot{\rho}_{\bar{q}}+\omega_{p}^{2} \rho_{\bar{q}}=- & -\sum_{i=1}^{N}\left(\frac{\vec{q}^{\prime} \cdot \vec{p}_{i}}{m}+\frac{\hbar q^{2}}{2 m}\right)^{2} \exp \left(-i \vec{q} \cdot \vec{n}_{i}\right)- \\
& -\sum_{\vec{q}^{\prime} \neq \vec{q}^{\prime}} \frac{\overrightarrow{q^{\prime}} \cdot \vec{q}^{\prime}}{m} \phi\left(\vec{q}^{\prime}\right) \rho_{\vec{q} \cdot \vec{q}^{\prime}} \rho_{\vec{q}^{\prime}}
\end{aligned}
$$


31

Novamente separamos o termo RPA e a correção SCFA, respectivamente o primeiro e o segundo somatōrios do lado direito. Se o lado direito da equa ção for nulo, a matriz densidade $\rho_{\vec{q}}$ vai oscilar com a frequência de plasma $\omega_{\mathrm{p}}$

$$
\begin{aligned}
& \text { Usando a expressão (2.19), podemos obter: } \\
& \rho_{\bar{q}^{-\vec{q}^{\prime}}} \rho_{\vec{q}^{\prime}}=\left(\sum_{j=1}^{N} e^{\left.-i\left(\vec{q}-\vec{q}^{\prime}\right) \cdot \vec{r}_{j}\right)}\right)\left(\sum_{i=1}^{N} e^{-i \vec{q}^{\prime} \cdot \vec{r}_{i}}\right)= \\
& =\sum_{i=1}^{N} e^{-i \vec{q} \cdot r_{i}} \sum_{j=1}^{N} e^{-i\left(\vec{q}^{\prime}-\vec{q}\right) \cdot\left(\vec{r}_{i}-\vec{r}_{j}\right)}=\rho_{\vec{q}} S\left(\vec{q}-\vec{q}^{\prime}\right)
\end{aligned}
$$

onde substituímos o último somatōrio sobre o indice $j$ por sua média estática nas $\mathrm{N}$ partículas .

Dessa forma, a equação de movimento para $\rho_{q}^{\rightarrow}$ fica

$$
\ddot{\rho}_{\vec{q}}=-\sum_{i=1}^{N}\left(\frac{\vec{q} \cdot \vec{p}_{i}}{m}+\frac{\hbar q^{2}}{2 m}\right)^{2} \exp \left(i \vec{q} \cdot \vec{r}_{i}\right)-\psi(\vec{q}) \frac{q^{2}}{m} N \rho_{\vec{q}},
$$

onde

$$
\psi(\vec{q})=\phi(\vec{q})+\frac{1}{N} \sum_{\bar{q}^{\prime}} \phi\left(\vec{q}^{\prime}\right) \frac{\vec{q} \cdot \vec{q}^{\prime}}{q^{2}}\left[S\left(\vec{q} \cdot \vec{q}^{\prime}\right)-1\right],
$$

e $\phi(\vec{q})$ é a transformada de Fourier do potencial de interação entre as partî culas.

No caso de potencial de interação Coulombiana, obtém-se, para o poten cial efetivo,

$$
\psi(\bar{q})=\phi(\bar{q})[1-G(\bar{q})],
$$


32

com

$$
G(\vec{q})=-\frac{1}{\rho} \sum_{\vec{q}^{\prime}} \frac{\vec{q} \cdot \vec{q}^{\prime}}{\left(q^{\prime}\right)^{2}}\left[S\left(\vec{q}-\vec{q}^{\prime}\right)-1\right] .
$$

A Eq. (2.22) é análoga à equação obtida na aproximação $R P A, \operatorname{com} \psi(\vec{q})$ no lugar do potencial de interação $\phi(\vec{q})$. Como no caso clássico, o resultado RPA para o gás de elétrons é obtido fazendo $G(\vec{q})=0$.

Então, o mesmo procedimento utilizado em RPA pode ser estendido para obter a expressão SCFA da susceptibilidade $\chi\left(\vec{q}, w^{*}\right)$ do gás, ou para a constan te dielētrica em gās de elétrons, bastando apenas substituir $\phi(\vec{q})$ por $\psi(\vec{q})$ nos resultados.

Obtēm-se assim, para a susceptibilidade, a mesma equação do caso clás sico

$$
X\left(\vec{q}, \omega^{*}\right)=\frac{X^{0}\left(\vec{q}, \omega^{*}\right)}{1-\psi(\vec{q}) X^{0}\left(\vec{q}, \omega^{*}\right)},
$$

sendo a susceptibilidade do gás livre dada por sua expressão quântica

$$
\chi^{0}\left(\vec{q}, \omega^{*}\right)=\frac{1}{\hbar} \sum_{\bar{p}, \sigma} \frac{n_{\vec{p}+\vec{q}, \sigma}-n_{\bar{p}, \sigma}}{\omega^{*}-\omega_{\bar{p}} \bar{q}},
$$

onde

$$
\hbar \omega_{\vec{p} \vec{q}}=\varepsilon_{\vec{p}+\vec{q}}-\varepsilon_{\vec{p}}=\frac{\hbar^{2}}{2 m}(\vec{p}+\vec{q})^{2}-\frac{\hbar^{2} q^{2}}{2 m} .
$$

Para a constante dielétrica $\varepsilon\left(\vec{q}, \omega^{*}\right)$, vamos obter também uma expressão 
análoga ao caso clássico

$$
\epsilon\left(\vec{q}, \omega^{*}\right)=1-\frac{\phi(\vec{q}) \chi^{0}\left(\vec{q}, \omega^{*}\right)}{1+[\phi(\vec{q})-\psi(q)] \chi^{0}\left(\vec{q}, \omega^{*}\right)} .
$$

0 esquema autoconsistente para a determinação das propriedades de um gás de elētrons com interação fica então fechado com as Eqs.(2.6), (2.16) e $(2.17)$.

\section{3 - RESULTADOS}

Singwi e seus colaboradores desenvolveram o método SCFA para aplicá-1o no gás de elétrons tridimensional. Em seu desenvolvimento, a expressão do potencial efetivo de interação $\psi(\vec{q})$ envolve explicitamente a transformada de Fourier do potencial Coulombiano $(\mathrm{Eq} \cdot(2.24))$. Entretanto, a mesma aproxima ção pode ser estendida para um gás com outro tipo de interaçăo, usłando-se a Eq. (2.12) para obter uma expressão para o potencial efetivo $\psi(\vec{q})$, sem supor uma expressão explícita para $\phi(\vec{q})$. Este tratamento pode ser utilizado mesmo em alguns casos nos quais o potencial de interação entre as partículas não possui transformada de Fourier, ou apresenta singularidade, como é o caso do potencial de um sistema composto de partículas impenetráveis. Esta aplica ção se constitui em parte de nosso trabalho a ser desenvolvido nos capítulos seguintes.

Os resultados da aplicação do método SCFA, como descrito anteriormente em um gás de elétrons tridimensional, na região de densidades metálicas, fó ram bastante satisfatórios ${ }^{(5)}$, especialmente com relação aos valores da fun ção correlação $g(\vec{r})$ para pequenas separações das partículas. As teorias RPA 
e Hubbard apresentam, nessa região, valores bastante negativos para $g(r)$, pa ra pequenos valores de $\vec{r}$. A teoria SCFA apresenta $g(\vec{r})$ com valores sempre positivos ou levemente negativos, quando os valores negativos são (em mödulo) cerca de 40 a 80 vezes menores do que os valores preditos por Hubberd e RPA, respectivamente.

Entretanto, para a compressibilidade do gás, a teoria SCFA, como des crita, apresenta valores negativos em densidades tais que $r_{s}>5$, o que também acontece com RPA e Hubbard. Este fato poderia sugerir que o gás de elétrons sofreria uma transição para o estado sólido, como discutido por Wigner ${ }^{(10)}$. Is to, entretanto, não acontece, como foi mostrado por Van Horn ${ }^{(11)}$ utilizando resultados obtidos, por Carr et al ${ }^{(12)}$. A não existência de metais alcal nos com $r_{s}>5$ nos parece indicar que estes sistemas seriam instáveis, justifí cando assim os valores negativos para a compressibilidade.

\section{4 - MODIFICAÇOES NA SCFA}

R. Lobo \& P. Antoniewicz ${ }^{(13)}$ ponderaram que, no limite de pequenos com primentos de onda $(\vec{q} \rightarrow \infty)$; considerar a função correlação estática dos pares em equilibrio $g^{\circ}(\vec{r})$ é uma boa aproximação para a função de correlação efetiva - que não acontece no limite $q \rightarrow 0$. Para obter uma expressão adequada no $1 \underline{i}$ mite de grandes comprimentos de onda, consideraram que existe uma flutuação de densidade no sistema, e então sugeriram para a função correlação efetiva a expansão até primeira ordem na flutuação de densidade

$$
g\left(\left|\bar{r}-\vec{n}^{\prime}\right|, n\right)=g^{0}\left(\left|\bar{r}-\vec{r}^{\prime}\right|, n\right)+\frac{\partial g^{0}\left(\left|\vec{n}^{n}\right| \bar{n}^{\prime}, n\right)}{\partial n}\left[f^{(1)}(n)-f_{0}^{(1)}(n)\right],
$$


onde $g^{0}(r, n)$ é a função correlação em equilỉbrio, e $f^{(1)}(r)$ a função distrị buição de uma partícula.

A derivada da função correlação em relação à densidade pode ser relą cionada com a sua derivada em relação ao argumento $r$, na presença de modos co letivos, usando-se o fato de que a densidade depende do inverso do cubo da distância entre as partículas, obtendo-se

$$
\frac{\partial}{\partial n} g^{0}(n, n)=-\frac{n}{3 n} \frac{\partial}{\partial n} g^{0}(n, n) \text {. }
$$

Usando as expressões $(2.27)$ e $(2.28)$ na expressão de $\psi(\vec{q})$ obtida da Eq.(2.12), e integrando por partes, o potencial efetivo autoconsistente pode ser escrito como:

$$
\Psi(\bar{q})=-\frac{4 \pi}{3 q^{3}} \int_{0}^{\infty} d r[\operatorname{sen}(q n)-q n \cos (q r)] g^{0}(r)\left[\frac{d \phi}{d r}-\frac{1}{2} r \frac{d^{2} \phi}{d r^{2}}\right],
$$

que é a expressão adequada, no caso clássico, para o limite de grandes comprí mentos de onda. No limite de grande vetor de onda, a expressão obtida por Singwi et al, utilizando para a função correlação a expressão de equilibrio, é correta ${ }^{(14)}$.

Em um trabalho mais recente, Vashishta \& Singwi ${ }^{(15)}$ sugerem uma modif $\underline{i}$ cação semelhante na SCFA, com o objetivo de forçā-1a a obedecer quase exata mente a regra de soma da compressibilidade. Tal modificação consiste essen cialmente em considerar a variação da função correlação dos pares em um campo externo fraco, através de sua expansão linear em termos do afastamento de equilïbrio. Considerando para $g\left(\vec{r}, \vec{r}^{\prime}, t\right)$ uma forma simétrica, tem-se 


$$
g(\vec{n}, \vec{n}, t)=g^{0}\left(\left|\vec{r}-\overrightarrow{n^{\prime}}\right|\right)+\frac{1}{2}[\delta n(\vec{n}, t)+\delta n(\vec{n}, t)] \frac{\partial}{\partial n} g^{0}\left(\left|\vec{n}-\vec{n}^{\prime}\right|\right),
$$

onde $\delta n \bar{e}$ a resposta estática de densidade, sendo $n+\delta n(\vec{r}, t)$ a densidade lo cal em $\vec{r}$.

Esta escolha para $\mathrm{g}\left(\overrightarrow{\mathrm{r}}, \overrightarrow{\mathrm{r}}^{\prime}, t\right)$ faz com que os resultados para a compres sibilidade, obtidos a partir da regra de soma e da definição termodinâmica,coin cidam.

Entretanto, no caso quântico, a correção de campo local è mais complí cada, e introduz-se um parâmetro a, a ser ajustado a posteriori. Para um gás de elétrons obtém-se

$$
G(\vec{q}, a)=\left(1+a n \frac{\partial}{\partial n}\right)\left\{-\frac{1}{n} \int \frac{d \vec{q}^{\prime}}{(2 \pi)^{3}} \frac{\vec{q} \cdot \vec{q}^{\prime}}{\left(q^{\prime}\right)^{2}}\left[S\left(\vec{q}-\vec{q}^{\prime}\right)-1\right]\right\},
$$

e o campo autoconsistente fica

$$
\psi(\vec{q}, a)=\phi(\vec{q})[1-G(\vec{q}, a)],
$$

dependendo de um parâmetro ajustável, ạ, que, como foi dito, será obtido ajus tando-se o cálculo da compressibilidade. 


\section{BIBLIOGRAFIA}

\section{CAPITULO 2}

1. D.Bohm \& D.Pines. Phys.Rev. 92, 609 (1953). Phys.Rev. 92, 626(1953). P.Nozieres \& D.Pines. Nuovo Cimento 9,470 (1958).

2. J.Hubbard. Proc.Roy.Soc. A 243, 336 (1957).

3. P.Noziēres \& D.Pines Phys.Rev. 111, 442 (1958).

4. K.S.Singwi, M.P.Tosi, A.Sj81ander Nuovo Cimento 54, B160 (1968).

*5. K.S.Singwi, M.P.Tosi, R.H.Land \& A.Sj8lander Phys.Rev. 176, 589 (1968).

6. D.Pines \& P.Nozières "The theory of quantum liquids" (W.A.Benjamin Inc., New York, 1966) Cap. 2 .

7. D.Pines "Elementary excitations in solids" (W.A.Benjamin Inc., New York, 1964) Cap. 3.

8. R.Brout \& P.Carruthers "Lectures on the many-electron problem" (Interscience Pub1., New York, 1963).

9. Por favor, veja Ref.7., pag. 98.

10. E.P.Wigner Trans.Far.Soc. 34, 678 (1938).

11. H.M.Van Horn Phys.Rev. 157, 342 (1967).

12. W.J.Carr, Jr., R.A.Coldwel1-Horsfall \& A.E.Fein Phys.Rev. 124, 747 (1961).

13. R.Lobo \& P.Antoniewicz Solid State Comm. 8, 461 (1970).

14. K.S.Singwi, K.SkH1d, M.P.Tosi Phys.Rev.Lett. 21, 881 (1968).

15. P.Vashishta \& K.S.Singwi Phys.Rev. B6, 875 (1972). e Phys.Rev. B6, 4883 (1972). 


\section{CAPITULO 3 \\ Aplicação de SCFA a um \\ sistema de bosons}

0 interesse no estudo das propriedades de um gās de bosons è em parte devido ao fato de que $4 \mathrm{He}$, a baixas temperaturas, pode ser estudado como um líquido quântico formado por um sistema de partículas interagentes que obede cem à estatística de Bose. O potencial de interação entre as partículas deve corresponder ao potencial interatômico em Hēlio. Têm sido propostas vărias expressões para representar a inter ação He-He, como é mostrado na Figura 1. Uma característica importante do potencial de interação ē a grande repulsão para pequenas separações de partículas, seguida de uma pequena atra ção em uma região correspondendo a maiores separações.

Como uma primeira aproximação vamos considerar ${ }^{4} \mathrm{He}$ como um sistema de bosons com potencial repulsivo tipo esfera dura ${ }^{(1,2)}$. Apōs, procurando uma melhor aproximação, vamos introduzir uma parte atrativa no potencial. Em am bos os casos, procuraremos obter o fator de estrutura $\mathrm{S}(\overrightarrow{\mathrm{q}})$, a função correla ção dos pares $g(\vec{r})$, o espectro de excitações elementares $E(\vec{q})$ e outras propriedades do gās, como velocidade do som, massa efetiva, etc.

\section{1 - METODO AUTOCONSISTENTE (SCFA)}

0 estudo de propriedades de ${ }^{4}$ He será feito utilizando-se uma adaptação do método SCFA desenvolvido para gás de eletrons, descrito no capítulo anterior.

Para um sistema de partículas eletricamente neutras, é conveniente es tudar-se a susceptibilidade do gás ou sua resposta ao campo externo aplicado. 
Assim, o conjunto de equações autoconsistentes a serem resolvidas deve envol ver o fator de estrutura $S(\vec{q})$, a função correlação $g(\vec{r})$, a susceptibilidade do gás considerado, $x\left(\vec{q}, \omega^{*}\right)$ e o potencial efetivo $\psi(\vec{q})$ que, em 1íquidos quân ticos, supõe-se ser independente da frequência.

0 fator de estrutura pode ser obtido a partir da susceptibilidade total do gās

$$
S(\vec{q})=-\frac{\frac{f}{h}}{\pi \rho} \int_{0}^{\infty} d \omega \operatorname{Im} X\left(\vec{q}, \omega^{*}\right)
$$

e a função de correlação estātica, de equilíbrio, dos pares é obtida da trans formada de Fourier de $S(\vec{q})$ :

$$
\begin{aligned}
& g^{0}(\vec{r})=1+\frac{1}{\rho} \int d \vec{q} e^{i \vec{q} \cdot \vec{r}}[S(\vec{q})-1], \text { ou } \\
& g^{0}(r)=1+\frac{2}{3 \pi} \frac{n_{0}^{3}}{r} \int_{0}^{\infty} d q g \operatorname{sen}(q r)[S(q)-1],
\end{aligned}
$$

onde $\rho$ é a densidade numérica das partículas do gás e $\mathbf{r}_{0}$,o raio da esfera equivalente ao volume médio ocupado por cada partícula, $\rho=3 /\left(4 \pi r_{0}^{3}\right)$. Por ou tro lado, na aproximação SCFA, a susceptibilidade do gās com interação é expressa como

$$
X\left(\vec{q}, \omega^{*}\right)=\frac{\chi^{0}\left(\vec{q}, \omega^{*}\right)}{1-\psi(\vec{q}) \chi^{0}\left(\vec{q}, \omega^{*}\right)},
$$

onde $\omega^{\star}=\omega+$ in é a frequência complexa, $\psi(\vec{q}) \bar{e}$ a interação efetiva autocon- 
sistente, a ser determinada, e $\chi^{0}\left(\vec{q}, w^{*}\right)$ é a susceptibilidade do mesmo gás sem interação.

Para um gás de Bose, em temperatura $\mathrm{T}=0^{\mathrm{O}} \mathrm{K}$, obtém-se para a susceptibi lidade do gás livre (vide apêndice A)

$$
\chi^{0}\left(\vec{q}, \omega^{*}\right)=\frac{2 \rho \varepsilon(\vec{q})}{\left(\hbar \omega^{*}\right)^{2}-[\varepsilon(\vec{q})]^{2}},
$$

onde $\varepsilon(\vec{q})=\not h^{2} q^{2} / 2 m$ è a energia de partícula ünica.

A susceptibilidade do gás com interação, usando as Eqs.(3.3) e (3.4), pode ser escrita como:

$$
\chi\left(\vec{q}, \omega^{*}\right)=\frac{2 \rho \varepsilon(\vec{q})}{\left(\hbar \omega^{*}\right)^{2}-[\varepsilon(\vec{q})]^{2}-2 \rho \varepsilon(\vec{q}) \psi(\vec{q})} .
$$

Desta expressão vemos que a susceptibilidade completa apresenta polos que nos vão permitir obter o espectro de energia da excitação.

A frequência $\omega_{o}(\vec{q})$, correspondente ao polo da susceptibilidade comple ta, em função do potencial efetivo $\psi(\vec{q})$, é obtida da solução da equação:

$$
\left[\hbar \omega_{0}(\bar{q})\right]^{2}-[\varepsilon(\vec{q})]^{2}-2 \rho \varepsilon(\vec{q}) \psi(\vec{q})=0
$$

e então a energia da excitação é dada por:

$$
E(\vec{q})=\hbar \omega_{0}(\vec{q})=\varepsilon(\vec{q}) \sqrt{1+\frac{2 p}{\varepsilon(\vec{q})} \psi(\vec{q})} .
$$


A susceptibilidade do gäs de bosons com interação fica:

$$
X\left(\vec{q}, \omega^{*}\right)=\frac{2 \rho \varepsilon(\vec{q})}{\left(\hbar \omega^{*}\right)^{2}-\left[\hbar \omega_{0}(\vec{q})\right]^{2}} .
$$

Assim, a parte imaginäria de $\chi\left(\vec{q}, w^{*}\right)$ pode ser escrita como

$$
\operatorname{Im}_{m} X\left(\vec{q}, \omega^{*}\right)=\frac{-4 \rho \varepsilon(\vec{q}) \hbar^{2} \omega \eta}{4(\hbar \omega)^{2}(\hbar \eta)^{2}+\left\{(\hbar \omega)^{2}-(\hbar \eta)^{2}-[E(\vec{q})]^{2}\right\}^{2}} .
$$

Usando as Eqs. (3.1) e (3.9), quando $n \rightarrow 0$, chega-se à expressão de Feynman para a energia de excitação de um sistema de bosons ${ }^{(\cdot)}$ :

$$
E(\vec{q})=\frac{\varepsilon(\vec{q})}{S(\vec{q})}
$$

o que permite escrever, para o fator de estrutura:

$$
S(\vec{q})=\left\{1+\frac{2 \rho}{\varepsilon(\vec{q})} \psi(\vec{q})\right\}^{-1 / 2} .
$$

Conhecendo-se a expressão para a interação efetiva $\psi(\vec{q})$, as Eqs. (3.7) e (3.11) permitem obter o espectro de excitações e o fator de estrutura para o sistema de bosons considerado.

o cálculo do potencial efetivo $\psi(\vec{q})$, é efetuado na aproximação SCFA, onde se obteve a relação

$$
\nabla_{\vec{r}} \psi\left(\vec{n}-\vec{r}^{\prime}\right)=g\left(\vec{n}-\vec{r}^{\prime}\right) \nabla_{\vec{r}} \phi\left(\vec{n}-\vec{r}^{\prime}\right),
$$

sendo $\phi\left(\vec{r}-\vec{r}^{\prime}\right)$ o potencial de interação entre os pares de partículas, e $g\left(\vec{r}-\vec{r}^{\prime}\right)$ a função correlação obtida a partir do fator de estrutura, pela e- 
quação $(3.2)$.

Obtém-se então para o potencial efetivo de interação $\psi(\vec{q})$ a expressão:

$$
\psi(\vec{q})=-\frac{4 \pi}{q^{3}} \int_{0}^{\infty} d r \frac{d \phi(r)}{d r} g(r)[\operatorname{sen}(q r)-q r \cos (q r)] .
$$

Para a função de correlação efetiva podemos usar a expressão proposta independentemente por R.Lobo \& P.Antoniewicz ${ }^{(4)}$ e Vashishta \& Singwi (5), que fazem uma expansão em torno da função de correlação estática de - equilíbrio $g^{\circ}\left(\left|\vec{r}-\vec{r}^{\prime}\right|\right)$ e consideram apenas os termos lineares na perturbação:

$$
g\left(\vec{r}-\vec{r}^{\prime \prime}\right)=g^{0}\left(\left|\vec{r}-\vec{r}^{\prime}\right|\right)+a \rho \frac{\partial g^{0}\left(\left|\vec{r}-\vec{r}^{\prime}\right|\right)}{\partial \rho},
$$

onde $\rho$ é a densidade numérica, e $\underline{a}$ um parâmetro a ser ajustado a posteriori, de forma a obedecer à regra de soma da compressibilidade.

A interação efetiva torna-se:

$$
\psi(\vec{q})=-\frac{4 \pi}{q^{3}} \int_{0}^{\infty} d r \frac{d \phi}{d r}[\operatorname{sen}(q r)-q r \cos (q r)]\left[g^{0}(r)+a \rho \frac{\partial g^{\circ}(r)}{\partial \rho}\right] \text {. }
$$

Então, o esquema autoconsistente é constituído das Eqs. (3.2), (3.11) e (3.12). E interessante ressaltar que, na obtenção das equações autoconsistentes, a única hipótese feita sobre o potencial entre as partículas, $\phi(\vec{r})$, é que ele deve ser derivável. 
3.2 - GATS DE BOSONS TIPO ESFERA DURA

Vamos supor que o gás de bosons seja constituído de esferas impenetráa veis de diâmetro $r_{c}$, com potencial de interação dado por:

$$
\phi(n)=\lim _{\lambda \rightarrow \infty} \lambda \theta\left(r_{c}-n\right),
$$

onde $\theta(r)$ è a função de Heaviside. 0 raio da esfera dura será deixado como parâmetro a ser ajustado a posteriori. Então, a derivada do potencial è dada pela função delta:

$$
\frac{d \phi}{d n}=\lim _{\lambda \rightarrow \infty}(-\lambda) \delta\left(r-r_{c}\right) \text {. }
$$

Com essa expressão para o potencial de interação, o potencial efetivo $\psi(\vec{q})$, expresso na Eq. (3.12) pode ser escrito como:

$$
\psi(\vec{q})=\frac{4 \pi r_{c}^{2}}{q} j_{1}\left(q r_{c}\right)\left[\lim _{\lambda \rightarrow \infty} \lambda g\left(r_{c}\right)\right],
$$

onde $j_{1}(x)=(\operatorname{sen} x-x \cos x) / x^{2}$ è a função de Bessel esfërica de primeira ordem.

Como o potencial $\psi(\vec{q})$ deve permanecer finito para todos os valores do vetor de onda $\vec{q}$, a função de correlação $g(\vec{r})$ deve se anular no ponto $r_{c}$, de forma a manter finito o produto $\lambda g\left(\mathrm{r}_{c}\right)$. Este resultado é consistente com o fato de que estamos considerando esferas totalmente rigidas, quando a menor distância possîvel entre duas partículas corresponde ao seu diâmetro. 
44

Como o produto $\lambda g\left(r_{c}\right)$ deve ser finito, podemos então introduzir uma constante $\mathrm{A}$, função da massa e das dimensões das partículas, tal que

$$
\lambda g\left(r_{c}\right) \equiv \frac{A \hbar^{2}}{12 m r_{c}^{2}} .
$$

0 fator $\hbar^{2} / 12 \mathrm{mr}_{\mathrm{c}}^{2}$ foi introduzido por conveniência futura. A constante A se rá determinada da condição $g\left(r_{c}\right)=0$. 0 potencial efetivo fica então

$$
\psi(\vec{q})=\frac{\pi \hbar^{2}}{3 m q} A j_{1}\left(q r_{c}\right) .
$$

Para o fator de estrutura, usando as Eqs. (3.11) e (3.19) e fazendo $x=\mathrm{qr}_{\mathrm{c}}$, ficamos com

$$
S(x)=\left\{1+A\left(\frac{r_{c}}{n_{0}}\right)^{3} \frac{j_{1}(x)}{x^{3}}\right\}^{-1 / 2}
$$

Para calcular o produto $A\left(r_{c} / r_{o}\right)^{3} \equiv B$, fazemos $g\left(r_{c}\right)=0$ na Eq. (3.2):

$$
g\left(n_{c}\right)=1+\frac{2}{3 \pi}\left(\frac{n_{0}}{r_{c}}\right)^{3} \int_{0}^{\infty} d x x \operatorname{sen} x[S(x, B)-1]=0
$$

ou

$$
1+\frac{2}{3 \pi}\left(\frac{n_{0}}{r_{c}}\right)^{3} T(B)=0,
$$

com 


$$
T(B)=\int_{0}^{\infty} d x \quad x \operatorname{sen} x[S(x, B)-1] .
$$

Vemos assim que, para cada valor da razão $\left(\mathrm{r}_{0} / \mathrm{r}_{c}\right)^{3}$, vamos obter um va lor para $\underline{A}$ através da Eq. (3.22). A Fig. 2 mostra a dependência de T(B) com o produto $B=A_{1}\left(r_{c} / r_{o}\right)^{3}$, obtida da Eq. (3.23). Então, para cada valor de $\left(r_{o} / r_{c}\right)^{3}$, obtém-se um valor de $\underline{A}$ que satisfaz à Eq. (3.22).

0 parâmetro $\underline{A}$ (ou $\underline{B}$ ) pode ser determinado independentemente, comparan do o máximo de $\mathrm{S}(x)$, dado pela Eq.(3.20), com o valor experimental do fator de estrutura do gás considerado $\left(T \approx 0^{\circ} \mathrm{K}\right)$. Obtém-se uma equação transcendental para $x_{0}$, posição do máximo de $\mathrm{S}(x)$, que não vai depender da constante A ou da razão $\left(\mathrm{r}_{\mathrm{o}} / \mathrm{r}_{\mathrm{c}}\right)^{3}$, isto é, independe do gás específico considerado,

$$
\tan x_{0}=\frac{5 x_{0}}{5-x_{0}^{2}} \text {, }
$$

cuja raĩz não nula vale $x_{0}=5.4486$.

A al tura do máximo, dada pelo valor $\mathrm{S}\left(x_{0}\right)$ na Eq.(3.20), vai depender apenas de $x_{o}$ e do produto $B=A\left(r_{c} / r_{o}\right)^{3}$, que pode então ser calculado a partir da comparação com resultados experimentais:

$$
S\left(x_{0}\right)=\left\{1+A\left(\frac{n_{c}}{n_{0}}\right)^{3} \frac{j_{1}\left(x_{0}\right)}{x_{0}^{3}}\right\}^{-1 / 2}=\left\{1-9.162 \times 10^{-4} \times A\left(\frac{n_{c}}{n_{0}}\right)^{3}\right\}^{-1 / 2} .
$$

Podemos então determinar tanto a constante $\underline{A}$ como a razão $\left(r_{o} / r_{c}\right)^{3}$, 
conhecendo o valor experimental do máximo de $S(x)$, para o gás específico con siderado, e usando a Eq. (3.22).

\subsection{1 - Espectro de energia}

0 espectro de energia das excitações elementares em um gás de Bose com interação,é obtido da Eq. (3.7) para um dado valor de $B=A\left(r_{c} / r_{o}\right)^{3}$. Espećtros de energia, para diversos valores de $B$, são mostrados na Fig. 3 . Para valores de $B>400$, que correspondem $a\left(r_{o} / r_{c}\right)^{3}<\pi / 2$, de acordo com a Eq. (3.22), aparece um comportamento de roton. Ā medida em que o valor de B cres ce, o que corresponde a menores valores de $\left(r_{o} / r_{c}\right)^{3}$, o mínimo de energia diminui, acentuando o roton. 0 valor crítico de $\left(r_{o} / r_{c}\right)^{3}$ pode ser obtido considerando-se estrutura tipo hcp, onde

$$
\left(\frac{n_{0}}{n_{c}}\right)^{3}=\frac{3}{\pi 2^{5 / 2}},
$$

o que nos $d \bar{a} T(B) \cong-28$ e $B \cong 1090$.

\subsection{2 - Fator de estrutura e função correlação dos pares}

0 fator de estrutura $S(x)$, para um dado valor de $B=A\left(r_{c} / r_{0}\right)^{3}$ é obtido usando-se a Eq.(3.20). A autoconsistência é garantida pela Eq.(3.21), que nos permite obter pares de valores de $\mathrm{A}$ e $\left(\mathrm{r}_{0} / \mathrm{r}_{c}\right)^{3}$ que satisfazem à condição $g\left(r_{c}\right)=0$.

Por outro lado, a função correlação dos pares $g(r)$, é obtida a partir do fator de estrutura autoconsistente, usando-se a Eq.(3.2).Para que o poten cial efetivo se mantenha finito, foi imposta a condição de que a função corre lação seja nula quando $r=r_{c}$. Vamos considerar então para 
$g(r)$ o resultado obtido a partir do valor autoconsistente de $S(x)$ apenas na região $r \geqslant r_{c}$. Para $r \leqslant r_{c}$, a hipótese de partículas impenetráveis faz com que se imponha que $g(r)=0$ nessa região.

\section{2 .3 - Velocidade do som}

Para um gás de partículas de massa $m$, com densidade numérica $\rho$, a regra de soma da compressibilidade pode ser escrita como

$$
\lim _{\vec{q} \rightarrow 0} \chi(\vec{q}, 0)=-k \rho^{2}=-\frac{\rho}{m s^{2}},
$$

onde $K \bar{e}$ a compressibilidade do sistema e $s$ a velocidade do som.

Usando a expressão obtida para a susceptibilidade do sistema de bosons, Eq. (3.8), chega-se a

$$
\chi(\vec{q}, 0)=-\frac{2 \rho \varepsilon(\vec{q})}{[E(\vec{q})]^{2}},
$$

o que nos permite escrever para a velocidade do som

$$
S=\lim _{q \rightarrow 0} \frac{E(q)}{\hbar q},
$$

e para o sistema de bosons com interação tipo esfera dura

$$
S=\frac{\hbar}{2 m r_{c}}\left(\frac{A}{3}\right)^{1 / 2}\left(\frac{r_{c}}{r_{0}}\right)^{3 / 2} .
$$

Supondo-se gás extremamente diluído, $\left(\mathrm{r}_{\mathrm{o}} / \mathrm{r}_{\mathrm{c}}\right)^{3} \rightarrow 0$, obtém-se $A=18 \mathrm{e}$ 
para a velocidade do som

$$
S=\frac{\hbar}{m n_{c}} \sqrt{\frac{3}{2}}\left(\frac{r_{c}}{r_{0}}\right)^{3 / 2},
$$

que é o resultado obtido por R.Lobo \& P.Antoniewicz ${ }^{(1)}$. Esse resultado difere do resultado exato ${ }^{(6)}$ por um fator $1 / \sqrt{2}$, que pode ser justificado por que, ao considerarmos baixas energias, o diâmetro efetivo é duas vezes maior que o diâmetro da esfera dura.

\subsection{4 - Massa efetiva}

Para definir $m^{*}$, a massa efetiva do roton, faz-se uma comparação entre a expansão da energia em torno do vetor de onda $q_{0}$ correspondente ao roton e a energia de uma partícula única com massa m*:

$$
E(q) \equiv E\left(q_{0}\right)+\frac{\hbar^{2}\left(q-q_{0}\right)^{2}}{2 m^{*}} \cong E\left(q_{0}\right)+\left.\frac{\left(q-q_{0}\right)^{2}}{2} \frac{d^{2} E}{d q^{2}}\right|_{q=q_{0}}
$$

Então

$$
\frac{1}{m^{*}}=\left.\frac{1}{\hbar^{2}} \frac{d^{2} E}{d q^{2}}\right|_{q=q_{0}}
$$

A expressão de Feynman (3.10) permite escrever $E(q)$ em função do fator de estrutura $S(q)$. Usando esse resultado, e a condição de que $q_{o} \bar{e}$ ponto de minnimo da energia, obtēm-se

$$
\left.\frac{d^{2} E}{d q^{2}}\right|_{q=q_{0}}=\frac{\hbar^{2}}{m S\left(q_{0}\right)}\left\{1-\left.\frac{q_{0}^{2}}{2 S\left(q_{0}\right)} \frac{d^{2} S}{d q^{2}}\right|_{q=q_{0}}\right\},
$$


e, fazendo $x=\mathrm{qr}_{c}$ :

$$
\frac{m}{m^{*}}=\frac{1}{S\left(x_{0}\right)}-\left.\frac{x_{0}^{2}}{2\left[S\left(x_{0}\right)\right]^{2}} \frac{d^{2} S}{d x^{2}}\right|_{x=x_{0}} .
$$

3.2 .5 - Comparação $\mathrm{com}^{4} \mathrm{He}$

Para verificar a concordância dos resultados teóricos obtidos para um sistema de bosons com interação tipo esfera dura, vamos compará-1os com os resultados experimentais para o gās de ${ }^{4} \mathrm{He}$, a baixas temperaturas $(7,8)$.

De acordo com a Eq.(3.24), a posição do máximo do fator de estrutura teórico não vai depender dos parâmetros $\underline{A}$ ou $\left(r_{0} / r_{c}\right)^{3}$ e é dada por $x_{0}=5.4486$, que corresponde ao valor experimental $q_{o} \sim 2.05 \AA^{-1}$ quando $r_{c} \simeq 2.6 \AA$. Esse valor é razoável para um modelo de ${ }^{4}$ He formado por partículas totalmente impenetráveis.

Por outro lado, a Eq. (3.25) permite obter o valor do produto $A\left(r_{c} / r_{o}\right)^{3}$ a partir do valor do máximo da curva experimental de $\mathrm{S}(x)$ e de sua posição:

$$
B=A\left(\frac{n_{c}}{n_{0}}\right)^{3}=\frac{x_{0}^{5}}{\operatorname{sen} x_{0}-x_{0} \cos x_{0}}\left[\frac{1}{\left[s\left(x_{0}\right)\right]^{2}}-1\right] .
$$

Usando o valor $\mathrm{S}^{\exp }\left(x_{0}\right) \cong 1.5$, obtém-se $\mathrm{B} \cong 607.5$.

Na Fig. 4 é feita a comparação entre a curva experimental e o resulta do teórico para o fator de estrutura calculado com $B=607.5$. Por outro lado, a Eq. (3.23) permite determinar o valor de $T(B)$ neste caso, e utilizando 
a Eq. (3.22), obtemos $\left(\mathrm{r}_{\mathrm{o}} / \mathrm{r}_{\mathrm{c}}\right)^{3}=1.234$, o que vai significar que $\mathrm{A}=749.6$. A densidade numérica do sistema de bosons descrito corresponde, então, a $\rho=1.10 \times 10^{22} \mathrm{~cm}^{-3}$, aproximadamente duas vezes mais diluído que $0^{4}$ He usual. Na Fig. 5 é mostrada a função correlação dos pares $g(r)$, obtida a partir do fator de estrutura autoconsistente. Para $r \leqslant r_{c}$, supomos que $g(r)=0$, concordando com a hipótese de impenetrabilidade das partículas.

Na Tabela I estão mostrados os valores para os parâmetros do nosso cál culo, obtidos por comparação com os resultados experimentais de ${ }^{4} \mathrm{He}$, a baixas temperaturas.

Tabela I

\begin{tabular}{|c|c|}
\hline $\mathrm{A}$ & 749.6 \\
$\left(\mathrm{r}_{\mathrm{o}} / \mathrm{r}_{\mathrm{c}}\right)^{3}$ & 1.234 \\
$\mathrm{~B}=\mathrm{A}\left(\mathrm{r}_{\mathrm{c}} / \mathrm{r}_{\mathrm{o}}\right)^{3}$ & 607.5 \\
$\mathrm{r}_{\mathrm{c}}$ & $2.6 \AA$ \\
$\mathrm{r}_{\mathrm{o}}$ & $2.8 \AA$ \\
\hline
\end{tabular}

O espectro de energia das excitações elementares correspondente a estes parâmetros, e o resultado experimental para ${ }^{4}$ He, são mostrados na Fig. 6 observa-se que a posição do máximo e do mínimo coincidem, embora os valores teóricos sejam maiores que os experimentais. Os valores correspondentes ao primeiro máximo, $E_{A}$, e ao primeiro mínimo, $E_{B}$, estão assinalados na figura. 
Os resultados teöricos correspondentes à velocidade do som, massa efe tiva, valores do máximo e do mínimo de energia de um gás de ${ }^{4} \mathrm{He}$ diluído estão mostrados na Tabela II, comparados com os correspondentes valores experi mentais, obtidos para $\mathrm{T}=0.160 \mathrm{~K}$.

Tabela II

\begin{tabular}{|c|c|c|}
\hline & Experimental & \multicolumn{1}{|c|}{ Teórico } \\
\hline$\rho$ & $2.18 \times 10^{22 \mathrm{~cm}^{-3}(9)}$ & $1.10 \times 10^{22} \mathrm{~cm}^{-3}$ \\
$\mathrm{E}_{\mathrm{A}}$ & $13.7^{\circ} \mathrm{K}(7)$ & $25.9^{\circ} \mathrm{K}$ \\
$\mathrm{E}_{\mathrm{B}}$ & $8.7_{\mathrm{K}}^{\circ}(7)$ & $17.8^{\circ} \mathrm{K}$ \\
$\mathrm{E}_{\mathrm{A}} / \mathrm{E}_{\mathrm{B}}$ & 1.57 & 1.45 \\
$\mathrm{~s}$ & $237 \mathrm{~m} / \mathrm{s}(10)$ & $434 \mathrm{~m} / \mathrm{s}$ \\
$\mathrm{m} * / \mathrm{m}$ & $0.16(7)$ & 0.2 \\
\hline
\end{tabular}

3.3 - GAS DE BOSONS: ESFERA DURA E POTENCIAL ATRATIVO

Os resultados obtidos no estudo do gás de ${ }^{4}$ He como um sistema de esfẹ ras duras, nos encorajaram a continuar utilizando o mesmo método, porém procurando um potencial que se aproximasse mais do potencial real entre os átomos de $\mathrm{He}^{(11)}$.

Vamos, então, considerar agora o potencial de interação como sendo composto de uma parte tipo esfera-dura, impenetrável, para pequenas separações de partículas, e uma parte atrativa, favorecendo a localização de cada 
partícula em uma região pouco maior do que a esfera dura. Desta forma, o potencial considerado vai aproximar-se mais da interação real He-He. O desenvolvimento serā anālogo ao feito com potencial esfera-dura, descrito na seção 3.2 .

Vamos considerar um potencial de interação $\phi(r)$ do tipo

$$
\phi(r)=\lim _{\lambda \rightarrow \infty} \lambda \theta\left(r_{c}-r\right)+v(r) \theta\left(r-r_{c}\right),
$$

onde a primeira parcela à direita representa a esfera dura, e v(r) é a parte atrativa do potencial. Por agora não è necessārio supor uma forma específi ca para a parte atrativa, o que será feito ao compararmos com os resultados para ${ }^{4}$ He.

A condição de continuidade do potencial estā satisfeita para qualquer valor de $r_{c}$, devido à forma do potencial tipo esfera dura. Para a derivada do potencial vamos ter:

$$
\frac{d \phi}{d r}=[v(n)-\lambda] \delta\left(r-r_{c}\right)+\theta\left(r-r_{c}\right) \frac{d r}{d r},
$$

onde usamos a notação simplificada $\lambda \equiv \underset{\lambda \rightarrow \infty}{\lim } \underset{\lambda}{\lambda}$.

0. potencial efetivo $\psi(q)$, de acordo com a Eq.(3.12), fica:

$$
\psi(q)=\frac{4 \pi r_{c}^{2}}{q}\left[\lambda g\left(r_{c}\right)\right] j_{1}\left(q r_{c}\right)-\frac{4 \pi r_{c}^{2}}{q} g\left(r_{c}\right) v\left(r_{c}\right) j_{1}\left(q r_{c}\right)-F(q),
$$


53

onde

$$
F(q)=\frac{4 \pi}{q} \int_{n_{c}}^{\infty} d r g(r) r^{2} j_{1}(q n) \frac{d v}{d r}
$$

e $j_{1}(q r)$ é a função de Bessel esférica de primeira ordem, com $\lim _{x \rightarrow \infty} j_{1}(x)=0$.

0 potencial efetivo $\psi(q)$ deve permanecer finito para todo q. Então, devemos ter o produto $\lambda g\left(r_{c}\right)$ finito, jā que a segunda parcela é finita devi do às características de $g(r)$ e do potencial atrativo. A presença da função de Bessel esférica e da derivada da parte atrativa do potencial na terceira parcela garante que a integral seja sempre finita. Para que o produto $\lambda g\left(r_{c}\right)$ seja finito, como $\lambda \rightarrow \infty$, é necessário que $g\left(r_{c}\right)=0$. Novamente vamos usar em $\psi(q)$, a constante finita $\underline{A}$, definida pela Eq. (3.18).

Fazendo $x=\mathrm{qr}_{c}$, o potencial efetivo pode ser escrito como:

$$
\Psi(x)=P(x)-F(x),
$$

onde $P(x)$ é o potencial efetivo devido à parte de esfera dura, expresso por

$$
P(x)=\frac{\pi \hbar^{2} r_{c}}{3 m} A \frac{j_{1}(x)}{x},
$$

e $F(x)$ è a correção devida à parte atrativa:

$$
F(x)=\frac{4 \pi r_{c}^{4}}{x} \int_{1}^{\infty} d y g(y) y^{2} j_{1}(x y) V(y),
$$

com $y=r / r_{c}$ e $v(y) \equiv \frac{1}{r_{c}} \frac{d v(y)}{d y}$. 
Temos assim separadas, no potencial efetivo, a contribuição de esfera dura, $P(x)$ e aquela devida à parte atrativa, $F(x)$, que depende da função correlação dos pares $\mathrm{g}(\mathrm{y})$, para $\mathrm{y}>1$.

O fator de estrutura $S(x)$ é expresso pela Eq.(3.11) que, para o poten cial considerado, fica

$$
S(x)=\left\{1+A\left(\frac{n_{c}}{n_{0}}\right)^{3} \frac{j_{1}(x)}{x^{3}}-\frac{3 m}{\pi \hbar^{2} n_{c}}\left(\frac{n_{c}}{n_{0}}\right)^{3} \frac{F(x)}{x^{2}}\right\}^{-1 / 2},
$$

e a função correlação dos pares $g(y)$, para $y>1$,é obtida a partir do fator de estrutura autoconsistente, através da Eq. (3.2).

Então, as equações que vão formar o conjunto de equações a ser resolvido de maneira autoconsistente, são as Eqs. (3.2 ), e (3.36)-(3.39), juntamente com a condição $g\left(r_{c}\right)=0$. E interessante ressaltar que não foi feita hipōtese sobre a expressão do potencial atrativo $v(r)$, que vai aparecer na correção $F(q)$ apenas com sua derivada. O diâmetro da esfera dura, $r_{c}$ e den sidade do gás são deixados como parâmetros a serem ajustados, repetindo-se o cálculo com vários pares de valores.

o cālculo é iniciado supondo-se apenas o potencial tipo esfera dura, is to $\overline{\mathrm{e}}, \mathrm{F}(x)=0$, e calcula-se $\psi_{0}(x, A)$ e $\mathrm{s}_{\mathrm{o}}(x, \mathrm{~A})$, obtendo o valor do parâmetro $A$, que anula $g_{o}\left(r_{c}\right)$, para um certo par de valores $r_{o}$ e $r_{c} \cdot$ Os valores de $A, r_{o}$ e $r_{c}$, são usados para calcular $g_{o}(y)$, para $y \geqslant 1$, que serā utilizado no cālculo da primeira correção $F_{0}(x)$. Com esse resultado voltamos a calcular - potencial efetivo $\psi_{1}(x, A)$ e o fator de estrutura, $S_{1}(x, A)$ correspondentes a essa primeira correção, procurando o novo valor de A que faz com que $g_{1}\left(r_{c}\right)=0$. Obtemos então os valores correspondentes a $g_{1}(y)$, com y $\geqslant 1$, e 
podemos calcular a correção seguinte, $F_{2}(x)$. Esse processo iterativo é repe tido até que se obtenha ao final de dois ciclos sucessivos os mesmos valores para a função correlação $g(y), y \geqslant 1$, dentro de uma precisão escolhida.

Como neste caso $F(x)$ depende diretamente de $r_{c}$, vemos que não basta escolher uma razão $\left(\mathrm{r}_{o} / \mathrm{r}_{c}\right)^{3}$, como fizemos antes. Vamos ter então três parâmetros ( $A, r_{0}$ e $\left.r_{c}\right)$ a serem ajustados. Além do critério de autoconsistência, pode-se usar a comparação com resultados experimentais em um certo sistema de bosons como critērio para escolha desses parâmetros.

o cálculo autoconsistente pode ser efetuado para qualquer potencial atrativo conveniente ao sistema a ser estudado, jā que não é suposta forma específica para $v(r)$. Como é nossa intenção comparar os resultados desta aproximação com um gās de ${ }^{4} \mathrm{He}$, vamos escolher para $v(r)$ expressões que descrevam a forma da parte atrativa do potencial real He-He. Dentre as expressões que tem sido propostas, as mais amplamente aceitas são aquelas sugeridas por Slater-Kirkwood ${ }^{(12)}$ e Lennard-Jones (13), mostradas na Fig. 1.

A forma proposta por Slater-Kirkwood é a seguinte:

$$
v(n)=A_{1} e^{-\alpha r / \sigma}-A_{2}\left(\frac{\sigma}{r}\right)^{6},
$$

com

$$
\begin{aligned}
\alpha & =21.5 \\
\sigma & =4.64 \times 10^{-8} \mathrm{~cm} \\
A_{1} & =5.67 \times 10^{6} \circ_{\mathrm{K}} \\
A_{2} & =1.080^{\circ}
\end{aligned}
$$

0 potencial de Lennard-Jones, para ${ }^{4} \mathrm{He}$ é expresso por: 


$$
v(n)=4 \varepsilon\left[\left(\frac{\sigma}{n}\right)^{6}-\left(\frac{\sigma}{n}\right)^{12}\right],
$$

$$
\begin{aligned}
\operatorname{com} \varepsilon & =10.22^{\circ} \mathrm{K} \\
\sigma & =2.556 \times 10^{-8} \mathrm{~cm}
\end{aligned}
$$

Para encontrar o resultado autoconsistente para o fator de estrutura $S(x)$ e para a função correlação $g(r), \operatorname{com} r>r_{c}$, realizamos o cálculo autoconsistente com cada um dos potenciais descritos correspondendo à parte atra tiva $v(r)$, escolhendo värios pares de valores $r_{o}$ e $r_{c}$ Os resultados finais obtidos para o fator de estrutura não diferiram sensivelmente, quanto à for ma, para cada potencial estudado. Entretanto, o potencial tipo Slater-Kirkwood mostrou valores mais adequados para a velocidade do som, comparada com o resultado de ${ }^{4} \mathrm{He}$. Por esta razão, parece mais adequado escolher-se para $\mathrm{v}(\mathrm{r})$ a Eq. (3.40).

Vamos descrever a seguir, com mais detalhes, os cálculos efetuados con siderando, para a parte atrativa, potencial tipo slater-Kirkwood. Em um con junto de cálculos usamos para $r_{0}$ o valor que corresponde à densidade experi mental de ${ }^{4} \mathrm{He}, r_{0}=2.22 \AA$, variando os valores de $r_{c}$. Verificou-se que 0 problema apresenta solução para valores de $r_{c}$ entre 2.24 e $2.5 \AA$. A convergência é alcançada neste caso com cerca de 10 a 15 iterações. Algumas curvas de $S(q)$ obtidas neste cālculo estão mostradas na Fig. 7 . Repetimos o cāl culo, mantendo a razão $\left(r_{o} / r_{c}\right)^{3}$ constante, com valor igual ao obtido no caso de potencial tipo esfera dura, e usando pares de valores adequados para $r_{0} e$ $r_{c}$, com $r_{c}$ variando entre 2.0 e $3.0 \AA$. Neste caso, o problema apresenta solução para valores de $r_{c}$ maiores do que $2.3 \AA$. A Fig. 8 mostra algumas cur vas de $S(q)$ obtidas neste cálculo. 


\subsection{1 - Fator de estrutura e função correlação}

0s resultados para o fator de estrutura $S(q)$ e para a função correlação dos pares $g(r)$, para $r \geqslant r_{c}$, são obtidos do próprio cálculo autoconsisten te, Eqs. (3.39) e (3.2). Neste caso, a condição imposta é que $g(r)=0$,po rém os valores de $g(r)$ para $r \geqslant r_{C}$ vão entrar no cálculo, na obtenção da correção $F(q)$.

\section{3 .2 - Espectro de energia e velocidade do som}

0 espectro de energia das excitações elementares em um sistema de bosons, considerando interação tipo esfera dura com parte atrativa, é obtido a partir do resultado autoconsistente para o fator de estrutura, usando-se a relação de Feynman, Eq.(3.10), ou seja:

$$
E(x)=\varepsilon(x)\left\{1+\frac{2 \rho}{\varepsilon(x)}[P(x)-F(x)]\right\}^{1 / 2},
$$

onde $P(x)$ e $F(x)$ são expressos em (3.37) e (3.38).

Para a velocidade do som, de acordo com a Eq.(3.27), quando $F(x) \neq 0$, chega-se a

$$
S=\frac{\hbar}{2 m r_{c}}\left(\frac{r_{c}}{r_{0}}\right)^{3 / 2}\left\{\frac{A}{3}-\frac{4 m r_{c}^{3}}{\hbar^{2}} \Phi\right\}^{1 / 2},
$$

onde $\Phi=\int_{1}^{\infty} d y y^{3} g(y) V(y)$ depende de $V(y)$, a derivado do potencial atrativo. 
58

3.3.3 - Massa Efetiva

A massa efetiva do roton pode ser obtida em função do fator de estrutura autoconsistente a partir da Eq. (3.31):

$$
\frac{m^{*}}{m}=\frac{2\left[S\left(x_{0}\right)\right]^{2}}{2 S\left(x_{0}\right)-x_{0}^{2} S^{\prime \prime}\left(x_{0}\right)} .
$$

Usando para o fator de estrutura a expressão (3.11) com o potencial $\underline{e}$ fetivo dado pela Eq.(3.36), podemos obter uma relação para $x_{0}$, a posição do máximo de $\mathrm{S}(x)$ :

$$
5 x_{0} \Psi\left(x_{0}\right)=\frac{\pi \hbar^{2} r_{c}}{3 m} A \operatorname{sen} x_{0}-F_{1}\left(x_{0}\right) \text {, }
$$

com

$$
F_{1}\left(x_{0}\right)=4 \pi r_{c}^{4} \int_{1}^{\infty} d y y^{2} g(y) V(y) \operatorname{sen}\left(x_{0} y\right) .
$$

Vemos que, nesse caso, a posição do máximo de $S(\underline{x})$ vai depender da cons tante $A$ e de $r_{c}$.

Para a segunda derivada de $S(x)$, calculada no ponto $x_{0}$, chega-se à expressão:

$$
S^{\prime \prime}\left(x_{0}\right)=\frac{-3 m r_{c}^{2}}{2 \pi \hbar^{2} r_{0}^{3}}\left[\frac{S\left(x_{0}\right)}{x_{0}}\right]^{3} \xi\left(x_{0}\right),
$$

com

$$
\xi\left(x_{0}\right)=-x_{0} P\left(x_{0}\right)+\frac{3}{x_{0}^{2}}\left[F_{1}\left(x_{0}\right)-F_{2}\left(x_{0}\right)\right]-\frac{10}{x_{0}} \psi\left(x_{0}\right)
$$


onde

$$
F_{2}\left(x_{0}\right)=4 \pi r_{c}^{4} x_{0} \int_{1}^{\infty} d y y^{3} g(y) V(y) \cos \left(x_{0} y\right) \text {. }
$$

Com o valor autoconsistente obtido para a função correlação para $\mathbf{r} \geqslant \mathbf{r}_{c}$, considerando um certo conjunto de valores para $A, r_{0}$ e $r_{c}$, podemos obter o valor de $S^{\prime \prime}\left(x_{0}\right)$ e, consequentemente, o valor para a massa efetiva do roton. Este cālculo è bastante trabalhoso, jā que envolve vārias integrais numéricas. Por outro 1ado, podemos obter o valor para a massa efetiva através do ajuste da expressão (3.29) para o resultado numérico para a energia da excitação $E(q)$, na região próxima do mínimo correspondente ao roton.

\section{3 .4 - Comparação com ${ }^{4} \mathrm{He}$}

As vārias curvas autoconsistentes obtidas para o fator de estrutura $S(q)$, com cada um dos conjuntos de parâmetros, podem ser comparadas com o re sultado experimental para ${ }^{4} \mathrm{He}$, a baixas temperaturas. A curva teórica que melhor se ajusta ao resultado do fator de estrutura experimental, corresponde a um potencial atrativo tipo slater-Kirkwood, $\operatorname{com} A=846.7, r_{c}=2.58 \AA$ e $r_{0}=2.767 \AA$, como é mostrado na Fig. 9 . Na Fig. 10 temos a função correla ção $g(r)$, obtida autoconsistemente para $r \geqslant r_{c}$ Quando $r<r_{c}$, supomos $g(r)=0$, de acordo com a hipótese de potencial tipo esfera dura nesta região.

0 espectro de energia obtido a partir do fator de estrutura autocon sistente, $\operatorname{com} A=846.7, r_{c}=2.58 \AA$ e $r_{o}=2.767 \AA$, é mostrado na Fig. 11 , comparado com o resultado experimental para ${ }^{4}$ He. Também nesse caso temos pa ra o resultado teórico uma forma semelhante à do resultado experimental, com 
as posições de máximo e mínimo coincidentes. Entretanto, o grä́fico teórico apresenta valores maiores que o experimental em todo o espectro de ener gias.

Os resultados para a velocidade do som e massa efetiva do roton, calcu lados considerando-se parte atrativa para o potencial são sensivelmente melhores que aqueles obtidos apenas com potencial tipo esfera dura, como pode ser verificado na Tabela III.

Tabela III

\begin{tabular}{|l|l|l|l|}
\hline & Experimental (7) & $\begin{array}{c}\text { Teórico } \\
\phi_{\mathrm{HS}}(\mathrm{r})=\lambda \theta\left(\mathrm{r}_{\mathrm{c}}-\mathrm{r}\right)\end{array}$ & $\begin{array}{c}\text { Teórico } \\
\phi(\mathrm{r}){ }_{\mathrm{HS}}(\mathrm{r})+\mathrm{v}(\mathrm{r})\end{array}$ \\
\hline$\rho$ & $2.18 \times 10^{22} \mathrm{~cm}^{-3}$ & $1.10 \times 10^{22} \mathrm{~cm}^{-3}$ & $1.13 \times 10^{22} \mathrm{~cm}^{-3}$ \\
$\mathrm{E}_{\mathrm{A}}$ & $13.7^{\circ} \mathrm{K}$ & $25.9^{\circ} \mathrm{K}$ & $23.6^{\circ} \mathrm{K}$ \\
$\mathrm{E}_{\mathrm{B}}$ & $8.7 \mathrm{o}_{\mathrm{K}}$ & $17.8^{\circ} \mathrm{K}$ & $14.8^{\circ} \mathrm{K}$ \\
$\mathrm{E}_{\mathrm{A}} / \mathrm{E}_{\mathrm{B}}$ & 1.57 & 1.45 & 1.59 \\
$\mathrm{~S}$ & $237 \mathrm{~m} / \mathrm{s}$ & $434 \mathrm{~m} / \mathrm{s}$ & $356 \mathrm{~m} / \mathrm{s}$ \\
$\mathrm{m} * / \mathrm{m}$ & 0.16 & 0.2 & 0.14 \\
\hline
\end{tabular}




\section{BIBLIOGRAFIA}

\section{CAPITULO 3}

1. R.Lobo \& P.Antoniewicz Phys.Rev.Lett. 24, 1168 (1970).

2. R.Lobo Phys.Rev. B12, 2640 (1975).

3. R.P.Feynman Phys.Rev. 94, 262 (1954).

K.Huang "Statistical Mechanics" (J.Wiley, New York, 1963) p. 381.

4. R.Lobo \& P.Antoniewicz Solid State Comm. 8 , 461 (1970).

5. P.Vashishta \& K.S.Singwi, Phys.Rev. B6, 875 (1972). e Phys.Rev. $4883(1972)$.

6. K.Huang, "Statistical Mechanics" (J.Wiley, New York, 1963) p.409.

7. D.G.Henshaw \& A.D.B.Woods Phys.Rev. 121, 1266 (1961)

R.A.Cowley \& A.D.B.Woods Phys.Rev. Lett. 21, 787 (1968) e Can. J.Phys., 49, 177 (1971).

P.Martel, E.C.Svensson, A.D.B.Woods \& V.F.Sears J.Low Temp.Phys. 23, $285(1976)$.

E.K.Achter \& L.Meyer Phys.Rev. 188, 291 (1969).

8. A.Miller, D.Pines \& P.Nozières Phys.Rev. 127, 1452 (1962).

9. J.Wilks "The properties of liquid and solid helium" (Claredon Press,Oxford, 1967) p.666

10. K.R.Atkins \& C.E.Chase Proc.Phys.Soc. (London) A 64, 826 (1951). 
11. J.0.Hirschfelder, C.F.Curtiss \& R.B.Bird "Molecular theory of gases and liquids" (J.Wiley, New York, 1954) p. 196 e 1068.

12. J.C.Slater \& J.G.Kirkwood Phys.Rev. 37, 682 (1931).

13. Por favor, veja Ref. 11, p. 196. 


\section{CAPITULO 4}

Aplicação de SCFA a um

sistema de jermions

0 método autoconsistente (SCFA) de Singwi et al, desenvolvido originalmente para gás de eletrons, pode ser também estendido para sistemas de fermions em geral, por exemplo, um gás ${ }^{3}$ He. A extensão é feita de maneira se melhante ao caso de um sistema de bosons, levando-se em conta as diferenças fundamentais entre os dois sistemas.

Pretendemos desenvolver o método SCFA para um gás de fermions s-dimen sional com potencial de interação genérico e,após, aplicar o método para um potencial singular tipo "esfera dura", isto é, considerar partículas impenetráveis. Vamos verificar que a aplicação em um sistema tri-dimensional vai exigir aproximações numéricas bastante elaboradas para sua solução, o que se rá deixado para ser desenvolvido em trabalho futuro. Entretanto, paraum sis tema de fermions com menor dimensionalidade, o esquema de cálculo numérico en volvido é bastante mais razoável e sua resolução e a discussão dos resultados é objeto de parte deste trabalho. O gás de eletrons unidimensional será objeto de estudos no próximo capítulo.

\section{1 - METODO ALTOCONSISTENTE}

Vamos considerar um sistema s-dimensional composto de fermions não car regados eletricamente e estudar a resposta $\chi(\vec{q}, \omega)$ a um campo externo aplica do. Seja $\phi(\vec{r})$ a interação entre as partículas, suposta diferenciável ao me nos no sentido generalizado. De maneira anāloga ao desenvolvido na Seção 3.1, vamos chegar a um conjunto de equações a ser resolvido de maneira auto- 
consistente, envolvendo o fator de estrutura $S(\vec{q})$, a susceptibilidade do gás $x(\vec{q}, \omega)$ e o potencial efetivo autoconsistente $\psi(\vec{q})$.

A susceptibilidade do sistema com interação, na aproximação SCFA,ē ex pressa em termos da interação efetiva $\psi(\vec{q})$ e da susceptibilidade do gás sem interação $\chi^{\circ}(\vec{q}, \omega)$ :

$$
\chi\left(\vec{q}, w^{*}\right)=\frac{\chi^{0}(\vec{q}, w)}{1-\psi(\vec{q}) \chi^{0}(\vec{q}, \omega)},
$$

onde $\omega^{*}=\omega+$ in $\bar{e}$ a frequência complexa.

Por outro lado, a interação efetiva $\psi(\vec{q})$, em SCFA, $\bar{e}$ obtida conhecendose $\circ$ potencial de interação $\phi(\vec{q})$ e a função de correlação dos pares $g(\vec{r})$ :

$$
\nabla_{\vec{r}} \psi\left(\vec{r}-\vec{r}^{\prime}\right)=g\left(\vec{r}-\vec{r}^{\prime}\right) \nabla_{\vec{r}} \phi\left(\vec{r}-\vec{r}^{\prime}\right) .
$$

A função correlação $g(\vec{r})$, para um sistema s-dimensional, com densidade numérica $\rho$, está relacionada com a transformada de Fourier do fator de es trutura $S(\vec{q})$ :

$$
S(\vec{q})-1=\rho \text { T.F. }[g(\vec{n})-1]
$$

ou

$$
g(\vec{r})=1+\frac{1}{\rho(2 \pi)^{s}} \int d^{s} q e^{i \vec{q} \cdot \vec{r}}[S(\vec{q})-1],
$$

e o fator de estrutura $S(\vec{q})$, por sua vez, pode ser obtido a partir da suscep 
tibilidade do sistema:

$$
S(\vec{q})=-\frac{\hbar}{\pi \rho} \int_{0}^{\infty} d \omega \operatorname{Im} \chi\left(\vec{q}, \omega^{*}\right) .
$$

Desse modo, para resolver as equações (4.1) a (4.4) de maneira autoconsistente, precisamos apenas conhecer o potencial de interação $\phi(\vec{r})$ entre as partículas e a susceptibilidade $\chi^{\circ}(\vec{q}, \omega)$ do gās sem interação, com mesma massa, densidade e estatística do sistema considerado.

A susceptibilidade $\times\left(\vec{q}, \omega^{*}\right)$, expressa em (4.1), apresenta polos que vão corresponder à frequência das excitações elementares $\dot{\omega}_{0}(q)$. Então, no cālculo da integral para o fator de estrutura, Eq.(4.4), é necessārio levar esse fato em consideração, separando-se os termos correspondentes aos polos. Calculando a parte imagināria da susceptibilidade completa, chega-se a

$$
\operatorname{Im} X\left(\vec{q}, w^{*}\right)=\frac{\operatorname{Im} x^{0}(\vec{q}, w)}{\left[1-\psi(q) \operatorname{Re} X^{0}(q, w)\right]^{2}+\left[\psi(q) \operatorname{Im} x^{0}(q, w)\right]^{2}}
$$

Como é mostrado no Apêndice A, para um sistema de fermions s-dimensio nal existem duas frequências determinadas, que vamos chamar, por agora, de $\omega_{1}$ e $\omega_{2}$, tais que apenas para frequências $\omega \operatorname{com} \omega_{1} \leqslant \omega \leqslant \omega_{2}$ vamos ter $I_{m} x^{\circ}(q, w)$ não nulo. Desse modo, como os polos de $I_{m} x(\vec{q}, \omega)$ correspondem a frequências $\omega_{0}(q)$ para os quais se deve ter

$$
I_{m} x^{0}\left(\vec{q}, \omega_{0}(q)\right)=0
$$


e

$$
\Psi(\vec{q})=\frac{1}{\operatorname{Re} \chi^{0}\left(\vec{q}, w_{0}(q)\right)},
$$

devemos ter $\omega_{0}(q)<\omega_{1}$ ou $\omega_{0}(q)>\omega_{2}$. A expressão para o fator de estrutura fica:

$$
\begin{aligned}
& S(\vec{q})=-\frac{\hbar}{\pi \rho} \int_{\omega_{1}}^{\omega_{2}} d \omega \operatorname{Im} \chi(\vec{q}, \omega)+ \\
& +\frac{\hbar}{\rho} \frac{1}{[\psi(q)]^{2}}\left[\left|\frac{\partial \operatorname{Re} \chi^{0}(q, \omega)}{\partial \omega}\right|_{\omega=\omega_{0}(q)}\right]^{-1}
\end{aligned}
$$

Dessa forma, separamos no fator de estrutura a contribuição de partícula única, correspondente a frequências $\omega$ tais que $\omega_{1}<\omega<\omega_{2}$ e a contri buição dos modos coletivos, calculada a partir do polo da susceptibilidáde $\chi(\vec{q}, \omega)$. A energia das excitações pode ser obitida em função do potencial efetivo $\psi(\vec{q})$, usando-se a expressão Re $\chi^{0}\left(\vec{q}, \omega_{0}\right)$ na Eq. (4.7). Precisamos cal cular então o potencial efetivo $\psi(\vec{q})$. Isto é feito a partir da Eq.(4.2), pa ra um sistema s-dimensional, e chega-se a:

$$
\Psi(\vec{q})=\phi(\vec{q})+\frac{1}{\rho} \int \frac{d^{s} q^{\prime}}{(2 \pi)^{s}} \frac{\vec{q} \cdot \vec{q}^{\prime}}{q^{2}} \phi\left(q^{\prime}\right)\left[s\left(\vec{q}-\vec{q}^{\prime}\right)-1\right] .
$$

Entretanto, para potenciais singulares, esta equação não é definida e então é mais conveniente obter o potencial efetivo a partir da função correlação $g(\vec{r})$, us ando-se: 


$$
\psi(\vec{q})=-\frac{i \vec{q}}{q^{2}} \cdot \int d_{n}^{s} \quad g(\vec{r}) \nabla \phi(\vec{r}) \exp (-i \vec{q} \cdot \vec{r}) \ldots(4 \cdot 10)
$$

Nesta expressão, a função $g(\vec{r})$ deve contrabalançar a singularidade do potencial, isto é, na região onde $\phi(\vec{r})$ é muito grande, $g(\vec{r})$ deve ser bem pequeno, de modo a manter $\psi(\vec{q})$ finito.

\section{2 - GAS DE FERMI TRIDIMENSIONAL}

Vamos examinar um sistema de fermions tri-dimensional, supondo que as partículas que o compõem possam ser consideradas impenetráveis. Isso equivale a tratar as partículas como esferas rígidas, de diâmetro $r_{c^{*}} 0$ potencial pode ser expresso usando-se a função de Heaviside $\theta(r)$ :

$$
\phi(r)=\lim _{\lambda \rightarrow \infty} \lambda \theta\left(r_{c}-r\right),
$$

e sua derivada vale:

$$
\frac{d \phi}{d r}=\lim _{\lambda \rightarrow \infty}(-\lambda) \delta\left(r-r_{c}\right)
$$

A Eq.(4.10) para o potencial efetivo, no caso tri-dimensional, pode ser escrita como

$$
\Psi(\vec{q})=-\frac{4 \pi}{q} \int_{0}^{\infty} d r r^{2} \frac{d \phi(n)}{d r} j_{1}(q r) g(r),
$$


68

onde $j_{1}(q r)$ è a função de Bessel esférica de ordem 1 .

Supondo potencial tipo esfera dura, analogamente ao caso de bosons, ob tém-se:

$$
\psi(\vec{q})=\frac{4 \pi r_{c}^{2}}{q} j_{1}\left(q r_{c}\right)\left[\lim _{\lambda \rightarrow \infty} \lambda g\left(r_{c}\right)\right] \text {. }
$$

Novamente, para que $\psi(\vec{q})$ seja finito, devemos ter $g\left(r_{c}\right)=0$, o que nos permitirá escrever o potencial efetivo em função de uma constante $\underline{C}$, a ser determinada:

$$
\psi(\vec{q})=\frac{c}{q} j_{1}\left(q r_{c}\right) .
$$

A susceptibilidade de um gás de fermions sem interação, com densidade numérica $\rho$, pode ser escrita como

$$
X^{0}\left(\vec{q}, w^{*}\right)=\operatorname{Re} X^{0}(\vec{q}, \omega)+i J_{m} X^{0}(\vec{q}, \omega),
$$

com

$$
\begin{aligned}
\operatorname{Re} X^{0}(\vec{q}, \omega)=-\frac{3 \rho}{4 \epsilon_{F} q}\left\{2 q+\left[1-\left(\frac{w+q^{2}}{2 q}\right)^{2}\right] \log \left|\frac{w+\omega_{+}(q)}{\omega+\omega_{-}(q)}\right|+\right. \\
\left.+\left[1-\left(\frac{w-q^{2}}{2 q}\right)^{2}\right] \log \left|\frac{w-\omega_{+}(q)}{\omega-\omega_{-}(q)}\right|\right\},
\end{aligned}
$$




$$
\begin{aligned}
\operatorname{Im} \chi^{0}(\vec{q}, \omega) & =-\frac{3 \pi}{8} \frac{\rho}{\epsilon_{F}} \frac{\omega}{q} & & 0 \leq \omega<\left|\omega_{-}(q)\right| \\
& =-\frac{3 \pi}{8} \frac{\rho}{\epsilon_{F} q}\left[1-\left(\frac{\omega-q^{2}}{2 q}\right)^{2}\right] & & \left|\omega_{-}(q)\right| \leq \omega \leq \omega_{+}(q) \\
& =0 & & \omega_{+}(q)<\omega
\end{aligned}
$$

onde expressamos $\omega$ em unidades de $\varepsilon_{F} / h$. $\varepsilon_{F}$ a energia de Fermi,e $q$ em unidades de $q_{F}$ e definimos $\omega_{ \pm}(q)=q^{2} \pm 2 q$.

Então, usando as Eqs.(4.7) e (4.17) podemos, em princípio, obter a frequência das excitações $\omega_{0}(q)$ em função de $\psi(q)$ que, por sua vez, vai depender da constante $C$. No caso de gás de fermions tri-dimensional, não é possível obter uma expressão analítica para $\omega_{0}(\mathrm{q}, \mathrm{C})$, e a solução numérica é bastante complicada. A função $\omega_{0}(q)$ é necessária no cálculo da parte coletí va do fator de estrutura. Entretanto, a constante $C$ que aparece no potencial efetivo $\psi(q)$ só pode ser determinada após o cālculo de $S(q)$, utilizando se a condição $g\left(r_{c}\right)=0$, o que torna ainda mais elaborada a obtenção de $\omega_{o}(q, C)$. Também o cálculo da contribuição da partícula única no fator de es trutura, neste caso, envolve uma integral bastante complexa,que vai depender da frequência da excitação (ou do parâmetro C). Esperamos chegar à resolução desse problema em um trabalho futuro.

Para testar a aplicação de SCFA a um sistema de fermions, podemos con siderar um sistema unidimensional. A principal diferença surge do fato de a expressão de $\chi^{0}(\vec{q}, \omega)$ para um sistema unidimensional ser muito mais simples, permitindo obter-se uma expressão analítica para a frequência do polo da sus 
ceptibilidade completa.

4.3 - GAS DE FERMI UNIDIMENSIONAL

Vamos considerar um sistema de fermions unidimensional, onde $x_{0}$ distância média entre as partículas, isto é, a densidade numérica vale $\rho=1 / x_{0}$. Se considerarmos as partículas com spin, a relação entre o vetor de onda de Fermi $q_{F}$ e a densidade numérica, fica

$$
q_{F}=\frac{\pi}{2} \rho=\frac{\pi}{2 x_{0}},
$$

- que nos permite escrever para a energia de Fermi:

$$
\epsilon_{F}=\frac{\hbar^{2} q_{F}^{2}}{2 m}=\frac{\hbar^{2} \pi^{2} \rho^{2}}{.8 m}
$$

A hipōtese de interação tipo esfera dura, em um sistema unidimensio nal, significa considerar-se as partículas como barras rígidas, de compri mento $\ell$. Nesse caso, o potencial real entre as partículas pode ser escrito como

$$
\phi(x)=\lim _{\lambda \rightarrow \infty} \lambda[\theta(x+l)-\theta(x-l)] .
$$

A expressão de $\psi(q)$, em uma dimensão, fica, de acordo com a Eq.(4.10):

$$
\Psi(q)=-\frac{2}{q} \int_{0}^{\infty} d x \operatorname{sen}(q x) g(x) \frac{d \phi}{d x},
$$


71

e, usando-se a expressão para o potencial $\phi(x)$, chega-se a:

$$
\Psi(q)=\frac{2}{q} \operatorname{sen} q l\left[\lim _{\lambda \rightarrow \infty} \lambda g(l)\right] \text {. }
$$

Como $\lambda \rightarrow \infty$, para que o potencial efetivo $\psi(q)$ permaneça finito, deve mos ter $g(\ell)=0$, e então podemos definir

$$
\lambda g(l)=A \pi \epsilon_{F}=\frac{\pi \epsilon_{F}}{(\rho l)^{3}} B,
$$

onde $A\left(\right.$ ou B) è um parâmetro a ser determinado pela condição $g(\ell)=0$, e $\boldsymbol{E}_{\mathrm{F}}$, $\rho$ e $\ell$ foram introduzidos por conveniência futura.

0 potencial efetivo fica então:

$$
\Psi(q)=2 \pi \epsilon_{F} A \frac{\operatorname{sen} q l}{q} .
$$

A susceptibilidade do gás de fermions unidimensional, sem interação,em unidades usuais, como é mostrado no Apêndice A, vale

$$
\chi^{0}\left(q, \omega^{*}\right)=\operatorname{Re} \chi^{0}(q, \omega)+i \operatorname{Im}_{m} \chi^{0}(q, \omega),
$$

com

$$
\operatorname{Re} \chi^{0}(q, \omega)=\frac{\rho q_{F}}{4 \epsilon_{F} q} \log \left|\frac{\omega^{2}-\omega_{-}^{2}(q)}{\omega^{2}-\omega_{+}^{2}(q)}\right|
$$




$$
\begin{aligned}
I_{m} \chi^{0}(q, w) & =-\frac{\pi \rho q_{F}}{4 \epsilon_{F}} \frac{1}{q} & & \omega_{-}(q) \leq \omega \leq \omega_{+}(q) \\
& =0 & & \omega<\omega_{-}(q)
\end{aligned}
$$

onde as frequências

$$
W_{ \pm}(q) \equiv\left|\frac{\hbar q^{2}}{2 m} \pm \frac{\hbar q q_{E}}{m}\right|
$$

correspondem aos valores extremos da banda de energias de excitação de um par fermion-buraco com vetor de onda $q$.

A frequência $\omega_{o}(q)$ correspondente ao polo da susceptibilidade completa, como $j \bar{a}$ vimos, deve estar fora da região compreendida entre $\omega_{-}(q)$ e $\omega_{+}(q)$, e pode ser obtida usando as Eqs.(4.7) e (4.27). Para um gás unidimen sional chega-se a:

$$
\log \left|\frac{w_{0}^{2}-w_{-}^{2}(q)}{w_{0}^{2}-w_{+}^{2}(q)}\right|=\frac{2 \pi \epsilon_{F} q}{q_{F}^{2} \psi(q)} \equiv \alpha(q)
$$

ou

$$
\omega_{0}^{2}(q)=\frac{\omega_{-}^{2}-\omega_{+}^{2} e^{\alpha(q)}}{1-e^{\alpha(q)}}=\frac{\omega_{+}^{2}-\omega_{-}^{2} e^{-\alpha(q)}}{1-e^{\alpha(q)}} .
$$

Supondo potencial tipo esfera dura, $\psi(q)$ é expresso pela Eq.(4.25), e ficamos com

$$
\alpha(q)=\frac{q^{2}}{q_{f}^{2} A \operatorname{sen}(q l)}
$$

Nesse caso então pode-se obter uma expressão analítica para $\omega_{0}(q)$, que 
vai depender do parâmetro $\underline{A}$, mas pode ser calculada explicitamente, em função desse parâmetro.

0 espectro de energia das excitações elementares em um gás de fermions unidimensional é expresso por

$$
E(q)=\hbar \omega_{0}(q)=\hbar \sqrt{\frac{\omega_{-}^{2}-\omega_{+}^{2} e^{\alpha}}{1-e^{\alpha}}},
$$

e,usando a expressão (3.27) para a velocidade do som,obtemos

$$
s=\frac{\pi \rho \hbar}{2 m} \sqrt{1+A \pi \rho l}=v_{F} \sqrt{1+A \pi \rho l} .
$$

Usando a frequência $\omega_{0}(q)$ do polo da susceptibilidade pode-se obter uma expressão para o fator de estrutura $S(q)$, em termos do parâmetro $\underline{A}$ :

$$
S(q, A)=S_{1}(q, A)+S_{2}(q, A),
$$

onde a contribuição de partícula única vale

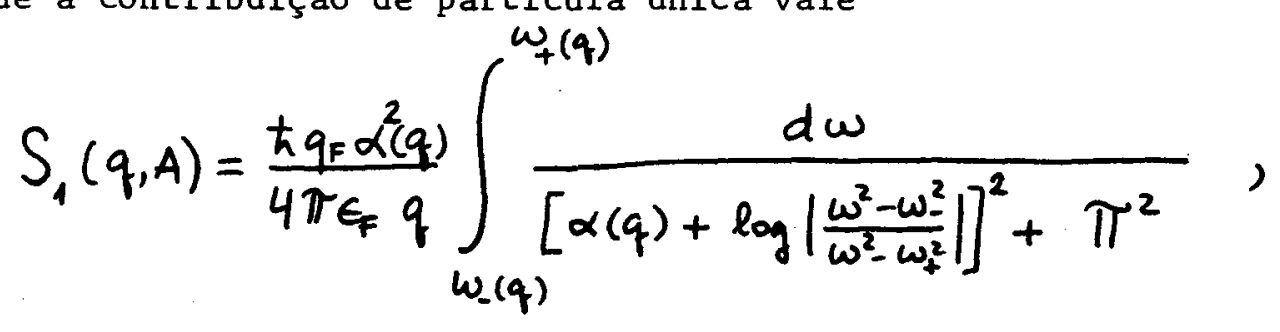

e a parte correspondente aos modos coletivos,

$$
S_{2}(q, A)=\frac{\hbar q_{F} \alpha^{2}(q)}{8 \epsilon_{F} q \omega_{0}} \frac{\left(\omega_{0}^{2}-\omega_{+}^{2}\right)\left(\omega_{0}^{2}-\omega_{-}^{2}\right)}{\left(\omega_{-}^{2}-\omega_{+}^{2}\right)} .
$$


A função correlação dos pares, em uma dimensão, é obtida do fator de estrutura, usando

$$
g(x)=1+\frac{1}{2 q_{F}} \int_{0}^{\infty} d q \cos q x[s(q)-1] .
$$

Por sua vez, o parâmetro $\underline{A}$ será obtido autoconsistentemente da condição

$$
g(l)=1+\frac{1}{\pi \rho l} T(A)=0
$$

$\operatorname{com}$

$$
T(A)=\int_{0}^{\infty} d(q l) \cos (q l)[S(q, A)-1]=-\pi \rho l .
$$

\section{4 - RESULTADOS}

Para obter as propriedades de um gás de Fermi unidimensional devemos então resolver de maneira autoconsistente as Eqs.(4.31), (4.35) e (4.37), jun tamente com a condição (4.38). Isto foi feito supondo vários valores para o produto $\rho \ell$, ou seja, variando a densidade do gás.

As equações do esquema autoconsistente podem ser reescritas em unidades adimensionais, fazendo $z=q / q_{F}, \quad y=x / l$ e $t=\pi \omega / \varepsilon_{F}$. Nestas uni dades podemos escrever

$$
\alpha(z)=\frac{z^{2}}{A \operatorname{sen}(C z)},
$$


onde $C=q_{F} \ell=\frac{\pi}{2}(\rho \ell)$, e as frequências $\omega_{+}(q)$ e $\omega_{-}(q)$ ficam:

$$
t_{ \pm}(z)=\left|z^{2} \pm 2 z\right|
$$

Para o fator de estrutura temos $S(z)=S_{1}(z)+S_{2}(z)$ com e

$$
S_{1}(z, A)=\frac{\alpha^{2}(z)}{4 z} \int_{t_{-}(z)}^{t_{1}(z)} \frac{d t}{\left[\alpha(z)+\log \left|\frac{t^{2}-t^{2}}{t^{2}-t_{+}^{2}}\right|\right]^{2}+\pi^{2}},
$$

$$
S_{2}(z, A)=\frac{\alpha^{2}(z)}{8 \epsilon_{F}} \frac{\left(t_{0}^{2}-t_{+}^{2}\right)\left(t_{0}^{2}-t_{-}^{2}\right)}{z t_{0}\left(t_{-}^{2}-t_{+}^{2}\right)},
$$

e para a função de correlação dos pares

$$
g(y)=1+\frac{1}{2} \int_{0}^{\infty} d z \quad \cos (C y z)[S(z, A)-1] .
$$

0 espectro de energia das excitações elementares fica

$$
E(z)=\epsilon_{F} \sqrt{\frac{t_{-}^{2}-t_{+}^{2} e^{\alpha}}{1-e^{\alpha}}} .
$$

\subsection{1 - Cálculo do parâmetro A}

Os valores de $T(A)$ que satisfazem à condição $g(\ell)=0$, isto é $T(A)=-\pi \rho l$ estão mostrados na Fig.12, em função do produto $B=A(\rho \ell)^{3}$. $A$ mesma figura representa também a dependência de $\rho l \operatorname{com} A(\rho l)^{3}$ e sua forma in dica que nosso cālculo não deve ser vālido para valores de $\rho \ell$ próximos da u- 
nidade, como serā discutido adiante. Na verdade, de acordo com a Fig.12, ve mos que devemos considerar apenas os valores de $\rho \ell \lesssim 0.7$. Para cada valor de $\rho \ell$ nesta região, podemos determinar o valor da constante $\underline{A}$ e calcular o fator de estrutura e o espectro de energia autoconsistentemente, através das Eqs. $(4.33)$ e $(4.35-36)$.

\subsection{2 - Espectros de energia}

A anālise dos espectros de energia en função do produto $\rho \ell$ permite d.stinguir dois tipos de comportamento, para diferentes valores de $\rho \ell$. Verifica-se que até um certo valor intermediário para a densidade, $\rho \ell \leqslant 0.22$, as curvas de energia das excitações elementares apresentam curvatura positiva, próximo à origem, enquanto que para densidades mais altas a orientação da cur vatura $\bar{e}$ invertida. Este comportamento estā exemplificado nas Fig. 13 el 14 , on de mostramos alguns espectros de energia, com vārias densidades. Podemos en tão distinguir regiões de alta e baixa densidade com comportamentos diferentes. Este fato é importante ao considerarmos a velocidade do som, onde as regiōes apresentam-se bem distintas. Como vemos nas Figs.13 e14, o espectro de energia apresenta descontinuidades, quando os modos coletivos encostam no espectro de partícula única. Os valores do vetor de onda e energia logo an tes da primeira descontinuidade são aproximadamente os mesmos para todas as densidades. Então, a orientação da concavidade próxima à origem determina a velocidade do som.

As descontinuidades que vão aparecer no espectro de energia de um gás de Fermi unidimensional podem ser entendidas examinando-se a equação de movi mento para as flutuações de densidade $\rho_{q} \cdot$ Em segunda quantização, a hamil to 
niana efetiva de um sistema de $\mathrm{N}$ partículas que obedecem $\bar{a}$ estatística de Fermi-Dirac, pode ser escrita como (1)

$$
H_{0}=\sum_{q} \epsilon_{q} c_{q}^{+} c_{q}+\frac{1}{2} \sum_{q} \psi(q)\left[\rho_{q} \rho_{q}^{+}-N\right],
$$

onde $\varepsilon_{q}$ é a energia de partícula única, $\psi(q)$ é o potencial efetivo, $c_{q}^{\dagger}$ e $c_{q}$ são os operadores de criação e destruição para as partículas do sistema e a matriz densidade $\bar{e}$ dada por $\rho_{q}^{+}=\sum_{p} C_{p+q}^{+} c_{p}$, com $\rho_{q}^{+}=\rho_{-q}$.

A relação de dispersão pode ser obtida da equação de movimento para as flutuações de densidade. Vamos calcular o comutador entre a hamiltoniana do sistema e o operador associado ao par partícula-buraco:

$$
\begin{aligned}
& {\left[H, c_{p+k}^{+} c_{p}\right]=\hbar \omega_{p k}^{0} c_{p+k}^{+} c_{p}-} \\
& -\frac{1}{2} \sum_{q} \psi(q)\left\{\rho_{q}^{+}\left(c_{p+k}^{+} c_{p+q}-c_{p+k-q}^{+} c_{p}\right)\right\}_{+},
\end{aligned}
$$

onde $\pi \omega_{p k}^{o}=\varepsilon_{p+k}-\varepsilon_{p}$ e $\{A, B\}_{+}$indica o anti-comutador.

A Eq. (4.46) mostra que a excitação de um único par está ligada com a excitação de dois pares, e sucessivamente, o que origina uma série de equações acopladas que não pode ser resolvida de maneira exata. A aproximação de ordem mais baixa para resolver essa série de equações consiste em desprezar os termos de interação, isto $\bar{e}$, supor $\psi(q)=0$. Nesse caso, obtemos a aproximação de partícula livre para o par fermion-buraco, que oscila com frequência 
$\omega_{\mathrm{pk}}^{o}$. Fazendo aproximação tipo RPA, que consiste em considerar apenas termos de interação com transferência de momentum igual ao momentum tik do par que se quer es tudar, obtém-se ${ }^{(2)}$ :

$$
\left.\left[H_{b}, c_{p+k}^{+} c_{p}\right]=\hbar \omega_{p k}^{0} C_{p+k}^{+} c_{p}-\psi(k)\left[n_{p+k}^{0}-n_{p}^{0}\right] \sum_{q} C_{q+k}^{+} C_{q}\right)
$$

onde $\eta_{k}^{o}=\bar{c}_{k}^{\dagger} \bar{c}_{k} \bar{e}$ o operador número de ocupação. Esta equação $\bar{e}$ linear nos operadores do par e pode ser resolvida.

Se $\omega_{k}^{*}=\omega_{k}+$ in é a frequência de oscilação, a relação de dispersão é obtida fazendo

$$
\left[H_{0}, c_{p+k}^{+} c_{p}\right]=\hbar \omega_{k}^{*} c_{p+k}^{+} c_{p}
$$

e chega-se então a

$$
1=\frac{\psi}{\hbar}(k) \sum_{p} \frac{n_{p}^{0}-n_{p+k}^{0}}{\omega_{k}-\omega_{p k}^{0}+i \eta}
$$

Examinando a expressão (4.48) vemos que as descontinuidades no espectro de energia vão corresponder a posições onde $\psi(q)$ troca de sinal, mantendo pequeno seu valor. Se $\psi(q) \rightarrow 0^{+}$, $\omega$ deve estar bem próximo à banda de par tícula ünica, com $\omega=\omega_{+}(q)+\Delta, \Delta \geq 0$. Da mesma forma, se $\psi(q) \rightarrow 0^{-}$, devemos ter $\omega=\omega_{-}(q)-\Delta^{\prime}, \operatorname{com} \Delta^{\prime} \geq 0$. Assim, quando o potencial, inicialmente positivo, vai diminuindo até trocar de sinal, a energia das excitações vai sofrer uma descontinuidade, passando do valor $\hbar \omega_{+}(q)$ para o valor $\hbar_{\omega}(q)$.

Para densidades $\rho \ell>0.23$, vamos ter um comportamento tipo roton no 
espectro de energia, com o mínimo acentuando-se quando a densidade cresce, porém permanecendo na mesma posição em relação a $q$, como è mostrado na Fig. 14. o espectro de energia, na região de altas densidades, apresenta um segundo mínimo para $q \ell>\pi$. $\AA$ medida que a densidade cresce esse mínimo decresce e para $\rho \ell \sim 0.66$ vai tocar o eixo horizontal $E(q)=0$. Podemos então definir um vetor de onda crítico $q_{c} \cong 0.05 \AA^{-1}, \operatorname{com} \lambda=2 \pi / q_{c}=1 / \rho_{c}$ expressando a periodicidade da rede. Com essa densidade, correspondente a um espaçamento médio entre as partículas $x_{0} \sim \frac{3}{2} l$, alcançamos um estado cristalino e não podemos prosseguir com o mesmo formalismo para densidades mais altas. 0 fato de o espectro de energia apresentar o valor $E_{i}\left(q_{c}\right)=0$ para um valor $q_{c} \neq 0$, mostra uma instabilidade do sistema quanto aos modos coletivos, sendo que o vetor de onda correspondente pode ser associado a uma periodicidade na rede.

\section{4 .3 - Velocidade do som}

Os valores correspondentes à velocidade do som em função da densidade obtidos na Eq.(4.34) estão mostrados na Fig.15. Estes valores concordam com os valores obtidos calculándo-se a inclinação, perto da origem, do espectro de energia.

Novamente é evidente a existência de duas regiões de densidade, com comportamento diferentes. A região de baixas densidades, com $\rho \ell \lesssim 0.22$, é uma região de interação fraca, onde se tem para a velocidade do som valores próximos à velocidade de Fermi, $s-v_{F} \cdot N a$ região de densidades mais altas, entretanto, temos $s \gg v_{F}$, correspondendo a um sistema com interação forte. A região de transição, onde a velocidade do som varia bastante com a densida de, corresponde exatamente aos valores de densidade onde a concavidade de 
$E(q)$ perto da origem muda de sinal.

4.4.4 - Fator de estrutura e função de correlação dos pares

0 fator de estrutura autoconsistente $S(q)$ está mostrado na Fig.16, enquanto que na Fig. 17 temos a função correlação $g(x)$ obtida a partir de $S(q)$ com o auxílio da Eq. (4.37). Novamente as duas regiões de densidade :apresentam comportamentos bem distintos. Para baixas densidades, tanto o fator de estrutura como a função de correlação aproximam-se assintoticamente do valor unitārio. Entretanto, na região de densidades mais altas, o fator de estrutu ra vai apresentar um mäximo tanto mais acentuado quanto mais alta a densidade e a função de correlação passa a oscilar em torno do valor unitário. Quando a densidade cresce, as oscilações de $g(x)$ tornam-se mais acentuadas e regulares, indicando a formação de uma estrutura tipo cristalina $(\rho \ell>0.66)$, como é evidenciado na Fig. 18 .

\section{5 - COMPARAÇAO COM OUTROS SISTEMAS UNIDIMENSIONAIS}

Os resultados obtidos para um sistema unidimensional de fermions com interação tipo esfera dura mostram que ele não se comporta muito distintamen te de um sistema de bosons com o mesmo tipo de interação( 3 ). Por outro 1a do, Girardeau ${ }^{(4)}$ mostrou que um sistema unidimensional de bosons puntuais e impenetráveis não difere sensivelmente de um sistema de fermions sem inte ração.

Considerando um sistema de fermions com spin, sem interação, o fator de estrutura pode ser calculado através da relação 
81

$$
S^{0}(q)=-\frac{\hbar}{\pi \rho} \int_{0}^{\infty} d \omega I_{m} X^{0}(q, \omega),
$$

que nos permite obter:

$$
\begin{aligned}
S^{0}(q) & =\frac{q}{2 q_{F}} & & q \leq 2 q_{F} \\
& =1 & & q>2 q_{F},
\end{aligned}
$$

e, para a função correlação dos pares, tem-se:

$$
g(x)=1-\frac{1}{2}\left[\frac{\operatorname{sen} q_{F} x}{q_{F} x}\right]^{2}
$$

0 resultado exato obtido por Girardeau, para um sistema unidimensional de bosons sem spin, considerados como partículas puntuais e impenetráveis é:

$$
g(x) \simeq 1-\left[\frac{\operatorname{sen} q_{x} x}{q_{x}}\right]^{2} .
$$

o fator $1 / 2$ não aparece devido a considerarmos partículas sem spin.

Para a velocidade do som o resultado exato de Girardeau,obtido a partir da energia do estado fundamental é:

$$
S_{\text {exato }}=\frac{v_{E}}{(1-\rho l)^{2}} \text {, }
$$




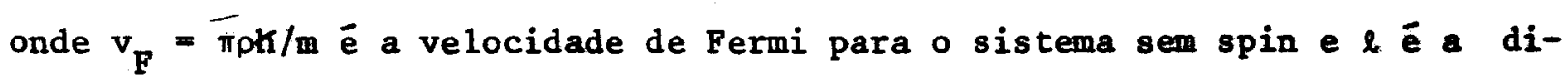
mensão da partícula impenetrável.

O nosso resultado- para a velocidade do som, usando SCFA para un sistema de fermions unidimensional, composto de barras rígidas de comprimento $\ell$ e considerando spin:é:

$$
S_{\text {SCFA }}=v_{F} \sqrt{1+A \pi \rho l},
$$

onde $v_{F}=\pi \rho \hbar / 2 m$, para partículas com spin. Como vimos, para baixas densida des a constante $\underline{A}$ cresce com $\rho \ell$. Então, no limite de partículas puntuais $(l \rightarrow 0)$, ambos os resultados coincidem, e temos $s \cong v_{F}$, que $\bar{e}$ o resultado p$\underline{a}$ ra o sistema sem interação.

A aproximação SCFA pode ser usada para estudar um gás de bosons impenetrāveis em uma dimensão ${ }^{(3)}$ de maneira análoga ao feito para o sistema tri dimensional. Examinando os resultados obtidos para a velocidade do som nesse caso, vemos que os valores concordam exatamente com aqueles que obtivemos para um sistema de fermions unidimensional, com mesma massa e mesmo tipo de interação. Também para o sistema de bosons, a aproximação SCFA só pode ser utilizada na região de densidades tais que $\rho \ell<0.7$. E interessante ressal tar que, para um sistema de bosons impeneträveis, em uma dimensão, podemos distinguir as duas regiões de densidades e o comportamento geral do espectro de energia é semelhante ao caso de fermions, principalmente para baixas densidades. Apenas, no caso de bosons, não vai aparecer a descontinuidade no espectro de energia, que é característica de um sistema de fermions,como foi mostrado. 


\section{BIBLIOGRAFIA}

\section{CAPITULO 4}

1. D.Pines "The many-body problem" (W.A.Benjamin, Inc., Reading, Mass. USA, 1962), p.42.

2. D.Pines em "Lectures on the many-body problem", vol. 2, E.P. Caianello,Ed. (Academic Press, New York, 1964), p.15.

3. O.Hipólito \& R.Lobo "Self-consistent field approximation method for a one-dimensional many-body system: Bosons", to be published.

4. M.Girardeau J.Math.Phys. 1,516 (1960). 


\section{CAPITULO 5}

\section{Gás de eletrons unidimensional}

Recentemente $(1,6)$ tem sido intensificado o interesse no estudo de condutores e semicondutores de um tipo particular, que exibem uma forte anisotropia, comportando-se praticamente como condutores unidimensionais. Um modelo satisfatório para esses materiais é supor que eles sejam compostos de "fios" condutores formados por cadeias de suas moléculas, ao longo dos quais os eletrons de condução se movem como em um condutor unidimensional. No modelo quasi-unidimensional supõe-se que os "fios" condutores são paralelos, suficientemente afastados de modo que se possa desprezar a interação entre eles.

As substâncias desse tipo que tem sido mais investigadas são compostos orgânicos de tetracyanoquinodimethane (TCNQ) com quinolinium ( $\left.Q_{n}-T C N Q\right)$, N-methylphenazinium (NMP-TCNQ) e tetrathiofulvalene (TTP-TCNQ) ${ }^{(3)}$, e compos tos inorgânicos como potassium-tetracyanoplatinates $(\mathrm{KCP})(\boldsymbol{H})$.

A teoria dos condutores lineares ou metais quasi-unidimensionais tem sido bastante desenvolvida recentemente. Entretanto a maioria dos trabalhos realizados refere-se ao cálculo das funções de onda. 0 estudo das excitações elementares em um gās de eletrons unidimensional foi feito usando o método de Bohm \& Pines (RPA), por värios autores $(5,6)$. Os resultados obtidos com RPA são satisfatórios na região de altas densidades, porém falham na região de densidades metálicas. A aproximação proposta por singwi et al (SCFA), apresentau sensivel melhora sobre os resultados de RPA para o gás de eletrons tridimensional. Nesta teoria são incluídas correções locais na inte 
ração, por meio de uma interação efetiva obtida considerando apenas contri buições de pares de particulas.

0 objetivo deste capítulo é aplicar a SCFA a um gás de elétrons consi derado como um sistema quasi-unidimensional, de maneira análoga ao desenvol vido no caso tridimensional, e comparar estes resultados com aqueles reporta dos na literatura.

\section{1 - METODO AUTOCONSISTENTE}

Vamos considerar o gás de elétrons como sendo composto de cadeias con dutoras unidimensionais e paralelas, ao longo das quais os elétrons se movem de maneira essencialmente unidimensional. Supõe-se que seja nula a probabi lidade de um elétron pular de uma cadeia para outra. Se $\Re$ é o número de fios condutores por unidade de área e se em cada um deles temos $\rho$ partículas por unidade de comprimento, o volume $V$ da célula unitária é $v=(\rho \boldsymbol{Z})^{-1}$. A interação coulombiana entre os pares de partículas em cada fio condutor é $\phi(x)$, e então o potencial efetivo em SCFA, $\psi(x)$, é obtido a partir da função de correlação dos pares $g(x)$, através da Eq.(2.12) para o caso unidimensio nal:

$$
\frac{d}{d x} \psi\left(x-x^{\prime}\right)=g\left(x-x^{\prime}\right) \frac{d}{d x} \phi\left(x-x^{\prime}\right) .
$$

Expressando as funções no espaço real em termos de suas transformadas de Fourier, e us ando

$$
\text { T.F. }[g(x)-1]=\frac{1}{\rho}[S(q)-1] \text {, }
$$


86

chega-se a

$$
\psi(q)=\phi(q)[1-G(q)]
$$

onde $\phi(q)=4 \pi e^{2} / q^{2} e$

$$
G(q)=-\frac{1}{4 q_{F}} \int_{-\infty}^{\infty} d q^{\prime} \frac{q}{q^{\prime}}\left[S\left(\left|\vec{q} \cdot \vec{q}^{\prime}\right|\right)-1\right] .
$$

A constante dielétrica $\varepsilon\left(q, \omega^{*}\right)$, dependente da frequência complexa

$\omega^{*}=\omega+$ in $e$ do vetor de onda $q$ pode ser obtida a partir da susceptibilidade $x\left(q, \omega^{*}\right)$ do gás com interação:

$$
\epsilon\left(q, w^{*}\right)=\frac{1}{1+\phi(q) \chi\left(q, w^{*}\right)}
$$

Na aproximação SCFA, a susceptibilidade do gás com interação é obtida em função do potencial efetivo autoconsistente $\psi(q)$ e da susceptibilidade $x^{0}\left(q, \omega^{*}\right)$ do mesmo gās sem interação

$$
\chi\left(q, w^{*}\right)=\frac{\chi^{0}\left(q, \omega^{*}\right)}{1-\psi(q) \chi^{0}\left(q, w^{*}\right)} .
$$

Então, para a constante dielétrica ficamos com

$$
\left.\epsilon\left(q, w^{*}\right)=1+\frac{Q_{0}\left(q, w^{*}\right)}{1-G(q) Q_{0}\left(q, w^{*}\right)}\right)
$$

onde $Q_{o}\left(q, w^{*}\right)=-\phi(q) x^{0}\left(q, w^{*}\right) \bar{e}$ a polarizabilidade dos eletrons livres, na direção das linhas condutoras. 
Usando o resultado obtido para $\chi_{1}^{0}\left(q, w^{*}\right)$ em um sistema unidimensional de fermions, no sistema quasi-unidimensional, a susceptibilidade do gás livre fica

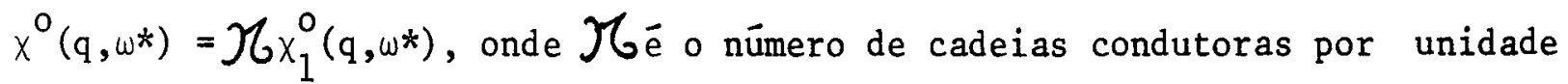
de ärea. Então, para a polarizabilidade do sistema quasi-unidimensional temos:

$$
Q_{0}\left(q, \omega^{*}\right)=\operatorname{Re} Q_{0}(q, \omega)+i \operatorname{Im} Q_{0}(q, \omega),
$$

com

$$
\begin{array}{rlrl}
\operatorname{Re} Q_{0}(q, \omega) & =\frac{1}{2} \frac{q_{F}}{q^{3}} q_{F T}^{2} \ln \left|\frac{\omega^{2}-\omega_{+}^{2}(q)}{\omega^{2}-\omega_{-}^{2}(q)}\right| \\
I_{m} Q_{0}(q, \omega) & =\frac{\pi}{2} \frac{q_{F}}{q^{3}} q_{F T}^{2} & & \omega_{-}(q) \leq \omega \leq \omega_{+}(q) \\
& =0 \quad & & \omega<\omega_{-}(q)
\end{array}
$$

onde $q_{F}$ é o vetor de onda de Fermi, $q_{F T} \bar{e} \circ$ análogo unidimensional do vetor de onda de Fermi-Thomas, $q_{F T}^{2}=m \omega_{p}^{2} / 2 \varepsilon_{F}$, e $\omega_{p} \bar{e}$ a frequência livre de plasma. Em um sistema quasi-unidimensional vale $\omega_{\mathrm{p}}^{2}=4 \pi \mathrm{e}^{2} / \mathrm{mV}$, V o volume da célula $\underline{\underline{u}}$ nitäria. As frequências

$$
\omega_{ \pm}(q)=\left|\frac{\hbar q^{2}}{2 m} \pm \frac{\hbar q q q}{m}\right|
$$


88

ção de um par eletron-buraco com vetor de onda $q$.

Por sua vez, o fator de estrutura $S(q)$ está relacionado com a constante dielétrica $\varepsilon\left(q, \omega^{*}\right)$ através da expressão:

$$
S(q)=-\frac{\hbar V}{\pi \phi(q)} \int_{0}^{\infty} d w I_{m}\left[\epsilon\left(q, w^{*}\right)\right]^{-1} .
$$

As equações $(5.4),(5.7)$ e (5.12), juntamente com a expressão $Q_{0}\left(q, \omega^{*}\right)$ constituem o esquema de equações a ser resolvido de maneira autoconsistente.

A expressão RPA para a constante dielētrica é

$$
\epsilon\left(q, w^{*}\right)=1-\phi(q) x^{0}\left(q, \omega^{*}\right)
$$

correspondendo então a uma aproximação de ordem zero em SCFA, com $G(q)=0$.

Usando a Eq. (5.7), podemos obter

$\operatorname{Im} \frac{1}{\epsilon(q, w)}=\frac{I_{m} Q_{0}(q, w)}{\left\{1+[1-G(q)] R_{e} Q_{0}(q, w)\right\}^{2}+\left\{[1-G(q)] \operatorname{Im} Q_{0}(q, w)\right\}^{2}} \cdot(14)$

Então, ao calcular o fator de estrutura, a integral vai ser efetuada entre os limites $\omega_{-}(q)$ e $\omega_{+}(q)$, quando $\operatorname{Im} Q_{0}(q, \omega)$ não é nulo. Entretanto, devemos também considerar a contribuição devida às frequências $\omega_{p}(q)$ tais que

Im $Q_{0}\left(q, \omega_{p}(q)\right)=0$

e 
89

$\operatorname{Re} Q_{0}\left(q, \omega_{p}(q)\right)=\frac{1}{G(q)-1}$.

os zeros da constante dielétrica vão corresponder aos polos de $\chi\left(q, w^{*}\right)$. Aplicando os resultados do caso unidimensional para um sistema quasi-dimensional, as frequências $\omega_{p}(q)$ são expressas por

$$
\omega_{p}^{2}(q)=\frac{\omega_{-}^{2}(q)-\omega_{+}^{2}(q) e^{\alpha(q)}}{1-e^{\alpha(q)}},
$$

onde

$$
\alpha(q)=\frac{\pi q^{3}}{q_{F}^{2} q_{F T}^{2} \vee[1-G(q)]} .
$$

0 fator de estrutura $\mathrm{S}(q)$ pode ser então obtido através da equação:

$$
\begin{aligned}
S(q) & =-\frac{\hbar V}{\pi \phi(q)} \int_{\omega_{-}(q)}^{\omega_{+}(q)} d \omega \operatorname{Im}\left[\epsilon\left(q, \omega^{*}\right)\right]^{-1}+ \\
& +\frac{\hbar V}{\phi(q)}\left[\left|\frac{\partial R_{e} \epsilon\left(q, \omega^{*}\right)}{\partial \omega}\right|_{\omega=\omega_{p}(q)}\right]^{-1},
\end{aligned}
$$

onde aparecem separadas a contribuiçaõ de excitação de partícula única e de excitação de plasmons.

A função de correlação dos pares $g(x)$ é obtida do fator de estrutura autoconsistente, usando 


$$
g(x)=1+\frac{1}{2 q_{F}} \int_{0}^{\infty} d q \cos q x[s(q)-1] \text {. }
$$

\section{2 - LIMITE DE GRANDES COMPRIMENTOS DE ONDA}

No limite de grandes comprimentos de onda, a função dielétrica quasiunidimensional na aproximação SCFA, que obtivemos na Eq.(5.7) satisfaz à con dição de blindagem perfeita, como se esperaria, isto é,

$$
\lim _{q \rightarrow 0} \frac{1}{\epsilon(q, 0)}=0
$$

\section{2 .1 - Relação de dispersão de plasmon}

Supondo frequências não nulas, a expressão da constante dielétrica,pạ ra pequenas transferências de momentum, fica:

$$
\lim _{q \rightarrow 0} \epsilon(q, \omega)=1-\frac{\omega_{p}^{2}}{\omega^{2}}\left[1+\left(\frac{q}{q_{F T}}\right)^{2}-\gamma\left(\frac{q}{q_{F}}\right)^{2}\right]+\ldots
$$

onde $\omega_{p}^{2}=4 \pi e^{2} / V m$ é a frequência livre de plasma e

$$
\gamma=\frac{1}{2 q_{F}} \int_{0}^{\infty} d q \frac{S(q)}{q^{2}} .
$$

Então, a relação de dispersão de plamons, para grandes comprimentos de onda, obtida dos zeros da constante dielétrica, pode ser escrita como 
91

$$
\omega_{p}(q)=\omega_{p}\left\{1+\left(\frac{q}{q_{F T}}\right)^{2}\left[1-\gamma\left(\frac{q_{F T}}{q_{F}}\right)^{2}\right]+\cdots\right\}^{1 / 2},
$$

ou, para grandes comprimentos de onda

$$
\omega_{p}(q)=\omega_{p}+\beta_{\text {SCFA }} \frac{\hbar q^{2}}{m}+\cdots,
$$

onde

$$
\begin{array}{ll}
\beta_{\text {SCFA }}=\beta_{\text {RPA }}\left[1-\gamma\left(\frac{q_{F I}}{q_{F}}\right)^{2}\right] \\
e \\
\beta_{\text {RPA }}=\epsilon_{F} / \hbar \omega_{P} .
\end{array}
$$

5.2 .2 - Compressibilidade

A razão $K_{\mathrm{f}} / K$ entre a compressibilidade de um gás livre unidimensio na1 e do mesmo gás com interação é obtida da regra de soma (7)

$$
\lim _{q \rightarrow 0} \epsilon(q, 0)=1+\left(\frac{q F r}{q}\right)^{2} \frac{k}{K_{f}} .
$$

Calculando o limite $q \rightarrow$ para a função dielétrica na aproximação SCFA, obtém-se

$$
\lim _{q \rightarrow 0} \in(q, 0)=1+\frac{\left(q_{F}+q\right)^{2}}{1-\gamma\left(q_{F} / q_{F}\right)^{2}}
$$

onde $\gamma$ foi definido na Eq. (5.23).

Para a razão das compressibilidades ficamos então com 


$$
\frac{k_{f}}{k}=1-\gamma\left(\frac{q_{F I}}{q_{F}}\right)^{2}=\frac{\beta_{S C F A}}{\beta_{R P A}} .
$$

\section{3 - RESULTADOS}

o cālculo autoconsistente para a obtenção das propriedades de um gás de eletrons quasi-unidimensional com interação coulombiana foi efetuado para vâa rios valores de $x_{S}=x_{0} / a_{0}$, onde $a_{0} \bar{e} \circ$ raio de Bohr e $x_{0}=1 / \rho$ a distância média entre as partículas na cadeia condutora. Para um sistema unidimensional, temos

$$
\rho=\frac{1}{x_{s} a_{0}}=\frac{2 q_{F}}{\pi},
$$

e então o vetor de onda de Thomas-Fermi pode ser expresso em função de $x_{s}$ :

$$
\left.\frac{q_{f T}^{2}}{q_{F}^{2}}=5.16 \times 10^{-17}\right)_{J} x_{s}^{3}
$$

Para o número de fios condutores por unidade de ārea usamos o valor $\zeta=10^{14} \mathrm{~cm}^{-2}$, que corresponde a uma distância média de $10 \AA$ entre os fios. A densidade volumétrica $N$ dos eletrons corresponde ao inverso do volume médio ocupado por cada eletron, is to $\bar{e}, N=1 / v=\varkappa_{\rho}$. Como usamos valores de $x_{s}$ entre 0.01 e 12 , vamos ter densidades volumétricas $\mathrm{N}$ entre $1.7 \times 10^{21}$ e $2 \times 10^{24} \mathrm{~cm}^{-3}$. No nosso cālculo, ao variarmos a densidade do gás, estamos considerando variações apenas na densidade dos eletrons em cada fio condutor, mantendo constante o nümero de fios condutores.

A aproximação SCFA, como jā vimos, consiste essencialmente em substi- 
tuir o potencial de interação $\phi(q)$ pelo potencial efetivo autoconsistente $\psi(q)=\phi(q)[1-G(q)]$. Iniciamos o cā1 culo supondo $G(q)=0$, obtendo então - resultado RPA para o fator de estrutura. Com esse resultado para $S(q)$, cal culamos a correção $G(q)$, e repetimos o processo de maneira autoconsistente, a té alcançarmos convergência com a precisão desejada. Para valores de $x_{s} a^{-}$ té 3 , a convergência foi alcançada com cerca de 5 iterações, enquanto que pa ra $3<x_{s}<7$, foram necessárias 12 iterações. O tipo de cálculo desenvolvi do só permite calcular até valores $x_{s}=12$, quando são necessárias cerca de 40 iterações para alcançar convergência.

\subsection{1 - Fator de estrutura e função correlação}

Os valores para o fator de estrutura $\mathrm{S}(\mathrm{q})$ foram obtidos autoconsisten temente, para vários valores da densidade $\rho$ de eletrons na cadeia condutora. Na Fig. 19 estão representados alguns resultados para $S(q)$, em função de $x_{s}$. Como se pode observar na figura, para $x_{s}=0.01$ o fator de estrutura apresen ta o valor correspondente ao gás unidimensional sem interação, Eq. (4.49).

A função de correlação dos pares para um gás de eletrons unidimensional é obtida a partir do fator de estrutura autoconsistente, através da Eq. (5.20). Nas Figs. 20 e 21 estão mostradas algumas curvas de $g(x)$ obtidas em SCFA, para vários valores de $x_{s}$, comparadas com os resultados de RPA. A medida ${ }^{\text {que }}$ a densidade cresce, os resultados obtidos com os dois métodos vão se aproximando e coincidem para $x_{s}=0.01$, que corresponde à região de altas densidades, onde se sabe que a aproximação RPA é satisfatória. Para valores 
$x_{s}>11$, a aproximação RPA origina resultados negativos para a função de cor relação $g(x)$ com pequenas separações das partículas. As características do cálculo desenvolvido neste trabalho não permitem obter resultados em SCFA Pạ ra $x_{s}>12$. Entretanto, como esses resultados ainda são bastante positivos, é razoável supor que a aproximação SCFA mantém $g(x)$ positiva para densidades bastante mais baixas em RPA.

\subsection{2 - Espectro de Energia e Compressibilidade}

As frequências dos modos coletivos $\omega_{\mathrm{p}}(q)$ foram calculadas para diver sos valores de $x_{\mathrm{s}}$ a partir dos zeros da constante dielétrica. Na Fig.22 te mos algumas curvas de dispersão, para vários valores da densidade. Para va lores de $x_{\mathbf{s}} \gtrsim 7$, os resultados de nosso cálculo indicam o aparecimento de um mínimo na energia de excitação dos modos coletivos para $\vec{q} \neq 0$, que vai se acentuando à medida que a densidade decresce. Esse resultado significa que, devido às correlações de curto alcance presentes no sistema, seria necessa rio menos energia para excitar um modo coletivo, no qual os elétrons tendem a ordenar-se, do que um modo coletivo de comprimento de onda infinito $(q=0)$. Esse resultado não é previs to em $R P A$.

Nas Fig.23 e 24, temos as curvas de dispersão em SCFA, comparadas com - resultado RPA e com a banda de excitação de partícula única,para vários vą lores de $x_{\mathrm{s}}$. Pode-se notar que as curvas de dispersão obtidas com RPA não apresentam mínimo, o que acontece na aproximação. SCEA,para baixas densidades. Novamente, para altas densidades, os resultados SCFA e RPA coincidem. Em am bas as aproximações, as curvas que representam as frequências das oscilações coletivas não cortam o espectro de partícula única, o que parece ser caracte 
rística do sistema unidimensional.

Os valores da função $\gamma$, obtidos a partir do fator de estrutura autoconsistente, através da Eq. (5.23), para diversas densidades, estão mostrados na Fig.25. Quando $x_{S} \rightarrow 0, \gamma$ cresce, porém o produto $\gamma\left(q_{F T} / q_{F}\right)^{2}+\overline{0}$ e en tão obtém-se o resultado RPA para a relação de dispersão de plasmons $\left(\beta_{\mathrm{SCFA}} \rightarrow \beta_{\mathrm{RPA}}\right):$

$$
\omega_{p}^{R P A}(q) \cong \omega_{p}\left(1+\frac{1}{2} \frac{q^{2}}{q_{F T}^{2}}\right)
$$

Na Fig. 26 estā representada a dependência da razão entre as compressi bilidades do gás livre e do gās com interação, em função de $x_{s}$. Na aproxí mação RPA a razão permanece constante e igual à unidade, porém na aproximação SCFA seu valor vai decrescendo quando a densidade decresce, alcançando va lores negativos para $x_{5} \geq 7.0$. 


\section{BIBLIOGRAFIA}

\section{CAPITULO 5}

1. H.G.Schuster, editor "One-dimensional conductors", German Physical Society Summer School Proceedings (Springer-Verlag, Berlin, 1975).

2. H.Kunz Annals of Phys. 85, 303 (1974)

V.J.Emery, A.Luther \& I.Peschel Phys.Rev. B13, 1272 (1976).

H.U. Everts, H.Shu1z Z.Physik B22, 285 (1975).

F.Devreux Phys.Rev. B13, 4651 (1976).

3. R.G.Kepler et al Phys.Rev.Lett. 5 , 503 (1960)

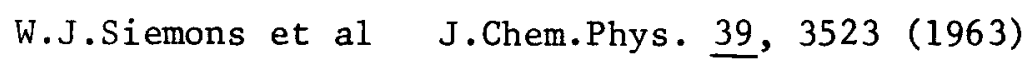

R.J.Birgeneau et a1 Phys.Rev.Lett. 26,718 (1971)

L.B.Cohen et a1 Solid State Comm. 12, 1125 (1973).

4. I.F.Schegolev Phys.Stat.Solidi A12, 9 (1972)

R.Renker et a1 Phys.Rev.Lett. $\underline{30}, 1144$ (1973) e Phys.Rev.Lett. $\underline{32}, 836$ (1974).

5. P.F.Williams \& A.N.Bloch Phys.Rev. B10, 1097 (1974)

6. M.Apostol Z.Physik B22, 279 (1975)
A.K.Das Solid State Comm. 15, 475 (1974).

7. D.Pines em "Lectures on the many-body problem", vol.2; E.P. Caianello, Ed. (Academic Press, New York, 1964), p.15. 
APENDICE A

Cálculo da susceptibilidade de um sistema de particulas livres : fermions e bosons

A.1 - SISTEMAS DE FERMIONS

Queremos calcular a susceptibilidade de um sistema de fermions sem interação, isto é, a resposta a uma excitaç-ao esterna suficientemente fraca para podermos usar aproximação linear. Vamos usar unidades adimensionais, expressando os vetores de onda em unidades de ${ }^{q_{F}} e$ as energias em unidades de $\varepsilon_{F}$.

Para um sistema com interação, obtivemos Eq. (1.11)

$$
\begin{aligned}
& \chi(\vec{q}, \omega)=\frac{1}{\epsilon_{F}} \sum_{n}\left|\left(\rho_{\vec{q}}^{+}\right)_{n_{0}}\right|^{2}\left\{\frac{1}{\omega-\omega_{n_{0}}+i \eta}-\frac{1}{\omega+\omega_{n o}+i \eta}\right\} \cdot(A .1) \\
& \text { Precisamos então calcular os elementos de matriz }\left\langle 0\left|\rho_{\vec{q}}^{+}\right| 0\right\rangle \text {, onde }
\end{aligned}
$$

$10\rangle$ representa o estado fundamental do sistema, com todos os estados de par tícula única até a superfície de Fermi preenchidos; $\rho_{\vec{q}}^{\dagger}=\sum_{\vec{p}} c_{\vec{p}+\vec{q}}^{\dagger} c_{\vec{p}}, c_{\frac{q}{q}}^{\dagger}$ e $c_{\vec{q}}$ são os operadores de criação e destruição de fermions. Estes elemen tos de matriz não são nulos apenas quando uma partỉcula de momentum $\overrightarrow{\mathrm{p}}$ foi excitada para um estado com momentum $\vec{p}+\vec{q}$, deixando um buraco em $\vec{p}$. Então, para um gás de fermions livres, a diferença de energia entre as duas configurações é

$$
\hbar \omega_{n 0}=\hbar \omega_{\vec{p} \vec{q}}^{0}=\epsilon_{\vec{p}+\vec{q}}^{0}-\epsilon_{\vec{p}}^{0}=q^{2}+2 \vec{q} \cdot \vec{p} .
$$

Assim, a susceptibilidade do gás livre pode ser escrita como 
98

$$
\begin{aligned}
& \chi^{0}(\vec{q}, \omega)=\frac{1}{\epsilon_{F}} \sum_{\substack{\vec{p}^{\prime} p^{\prime} \\
\sigma \sigma^{\prime}}}\left\langle 0\left|c_{\vec{p}^{\prime}-\vec{q} \sigma^{\prime}}^{+} c_{p^{\prime} \sigma^{\prime}} c_{\vec{p}+\vec{q} \sigma}^{+} c_{p \sigma}\right| 0\right\rangle \times \\
& x\left\{\frac{1}{\omega^{*}-\omega_{p \vec{q}}^{0}}-\frac{1}{\omega^{*}+\omega_{p \bar{q}}^{0}}\right\},
\end{aligned}
$$

com $\omega^{*}=\omega+i n$

Porém, temos

$$
\left\langle 0\left|C_{p^{\prime}-\vec{q} \sigma^{\prime}}^{+} C_{\vec{p}^{\prime} \sigma^{\prime}} C_{\vec{p}+\vec{q} \sigma}^{+} C_{\vec{p} \sigma}\right| 0\right\rangle=\delta_{\vec{p}^{\prime}, \vec{p}+\vec{q}} \delta_{\sigma \sigma^{\prime}},
$$

e assim, para a susceptibilidade, ficamos com

$$
\chi^{0}(\vec{q}, \omega)=\frac{1}{\epsilon_{F}} \sum_{\overrightarrow{p \sigma}}\left\langle 0\left|c_{\vec{p} \sigma}^{+} c_{\vec{p}+\vec{q} \sigma} c_{\vec{p}+\vec{q} \sigma}^{+} c_{\vec{p} \sigma}\right| 0\right\rangle\left\{\frac{1}{\omega^{*}-\omega_{p q}^{0}}-\frac{1}{\omega^{*}+\omega_{p \vec{q}}^{0}}\right\} .
$$

Os operadores de criação e destruição de fermìons obedecem às regras de anticomutação usuais:

$$
\begin{aligned}
& \left\{c_{\vec{q}}^{+}, c_{q^{\prime}}^{+}\right\}_{+}=\left\{c_{\vec{q}}, c_{q^{\prime}}\right\}_{+}=0 \\
& \left\{c_{\vec{q}}^{+}, c_{\vec{q}^{\prime}}\right\}_{+}=\frac{\delta_{q^{\prime}}}{}
\end{aligned}
$$

Usando as Eqs. (A.5) e (A.6), para $ఫ \neq 0$, podemos escrever

$$
\chi^{0}(\vec{q}, \omega)=\frac{1}{\epsilon_{F}} \sum_{\vec{p} \sigma}\left\langle 0\left|C_{\vec{p} \sigma}^{+} C_{\vec{p} \sigma}\left[1-C_{\vec{p}+\vec{q} \sigma}^{+} C_{\vec{p}+\vec{q} \sigma}\right]\right| 0\right\rangle\left\{\frac{1}{\omega^{*}-\omega_{\vec{p} \vec{q}}^{0}}-\frac{1}{\omega^{*}+\omega_{\vec{p}}^{0}}\right\} \cdot \text { (A.7) }
$$

Como o número de ocupação do estado com momentum $\overrightarrow{\mathrm{p}}$ é definido por

$$
\left\langle o\left|C_{\vec{p} \sigma}^{+} C_{\vec{p} \sigma}\right| 0\right\rangle=n_{\vec{p} \sigma}^{0},
$$


a Eq. (A. 7) fica

$\chi^{0}(\hat{q}, \omega)=\frac{1}{\epsilon_{F}} \sum_{\bar{p} \sigma} n_{\bar{p} \sigma}^{0}\left(1-n_{\vec{p}+\vec{q} \sigma}^{0}\right)\left\{\frac{1}{\omega-\omega_{\vec{p} \bar{q}}+i n}-\frac{1}{\omega+\omega_{\vec{p} \vec{q}}+i \eta}\right\}$.

Ou seja, $\chi^{0}(\vec{q}, \omega) \neq 0$ apenas quando $|\vec{p}|<q_{F}$ e $|\vec{p}+\vec{q}|>q_{F}$.

Podemos obter uma expressão equivalente para $\chi^{\circ}(q, \omega)$ se, no segun do termo da equação (A.9), trocarmos o indice mudo de soma $\vec{p} \rightarrow-\vec{p}-\vec{q}$. Des sa forma, tem-se $\omega_{\vec{p}} \vec{q} \rightarrow \omega_{\vec{p}} \vec{q}$, e então, como $n_{\vec{p}}=n_{-\vec{p}}$, podemos escrever

$$
\chi^{0}(\vec{q}, \omega)=\frac{1}{\epsilon_{F}} \sum_{\vec{p} \sigma} \frac{n_{\vec{p} \sigma}^{0}-n_{\vec{p}+\vec{q} \sigma}^{0}}{\omega-\omega_{\vec{p} \bar{q}}+i \eta}
$$

Ainda, se usarmos o fato de que, de acordo com a Eq. (A.8), temos

$$
\begin{aligned}
n_{\vec{p}}^{0} & =1 & \text { se } & |\vec{p}| \leq q_{F} \\
& =0 & \text { se } & |\vec{p}|>q_{F},
\end{aligned}
$$

e, fazendo uma nova mudança nos indices de soma, chega-se a

$$
\chi^{0}(\vec{q}, \omega)=\frac{1}{\epsilon_{F}} \sum_{\substack{\vec{p} \sigma \\|\vec{p}| \leq q_{F}}}\left[\frac{1}{\Omega_{+}+i \eta}-\frac{1}{\Omega_{-}+i \eta}\right] \text {, }
$$

onde $\quad \Omega_{ \pm}=\omega \pm q^{2}-2 \vec{q} \cdot \vec{p}$.

Para calcular a parte real e a parte imaginária de $\chi^{\circ}(\overleftrightarrow{q}, \omega)$, com 
100

$$
\chi^{0}(\vec{q}, \omega)=\operatorname{Re} \chi^{0}(\vec{q}, \omega)+i J_{m} \chi^{0}(\vec{q}, \omega)
$$

vamos usar a relação

$$
\lim _{\eta \rightarrow 0} \frac{1}{\Omega+i \eta}=P\left(\frac{1}{\Omega}\right)-i \pi \delta(\Omega),
$$

que vale rigorosamente dentro de uma integral.

A. .1 - Gás de fermions em uma dimensão

Para um sistema unidimensional de fermions com spin, temos

$$
\rho=\frac{N}{L}=\frac{2 q_{F}}{\pi} \text {. }
$$

Supondo uma distribuição contínua de momenta, em unidades adimensionais, va mos usar

$$
\sum_{\bar{p} \sigma} \longrightarrow \frac{\operatorname{Liq}}{2 \pi} \int d p=\frac{\rho}{4} \int d p,
$$

onde estamos supondo"volume" unitário. Para a parte real da susceptibilidade generalizada, usando Eq. (A.11), ob tem-se

$$
\operatorname{Re} \chi^{0}(q, \omega)=\frac{\rho}{4 G_{F} q} \log \left|\frac{\omega^{2}-\omega_{-(q)}^{2}}{\omega^{2}-\omega_{+}^{2}(q)}\right|
$$

$\operatorname{com} \omega_{ \pm}(q)=\left|q^{2} \pm 2 q\right|$.

Para calcular a parte imagináría é mais conveniente usar a Eq.(A.9), e então

$$
J_{m} \chi^{0}(q, \omega)=-\frac{\pi \rho}{4 \epsilon_{F}} \int d p \beta_{p q}\left[\delta\left(\omega-\omega_{p q}\right)-\delta\left(\omega+\omega_{p q}\right)\right],
$$


onde

$$
\beta_{q p}=\eta_{p}^{0}\left(1-n_{p+q}^{0}\right)=\left\{\begin{array}{lll}
1 & \text { se } & |\vec{p}| \leq q_{F} \leq|\vec{p}+\vec{q}| \\
0 & \text { se } & |\vec{p}|>q_{F} \text { ou }|\vec{p}+\vec{q}|<q_{F} .
\end{array}\right.
$$

Assim, podemos escrever para a parte imaginária da susceptibilidade

$$
J_{m} \chi^{0}(q, \omega)=-\frac{\pi \rho}{4 \epsilon_{F}} \int_{-q_{F}}^{q_{F}} d p[\delta(\omega-\omega)-\delta(\omega+\omega)],
$$

com a condição adicional $|\vec{q}+\vec{p}|>q_{F}$.

Efetuando a integral, supondo $\omega>0$, chega-se finalmente a

$$
\begin{aligned}
I_{m} \chi^{0}(q, \omega) & =-\frac{\pi \rho}{8 \epsilon_{F} q} & & \text { se } \omega_{-}<\omega<\omega_{+} \\
& =0 & & \text { se } \omega<\omega_{-} \text {oo } \omega>\omega_{+}
\end{aligned}
$$

onde $\quad \omega_{ \pm}=\left|q^{2} \pm 2 q\right|$.

As equações (A.13) e (A.16) permitem então obter-se a expressão completa para a susceptibilidade generalizada, em um gás ee fermions uni dimensional.

A.1.2 - Gás de fermions tridimensional

0 cálculo da susceptibilidade de um sistema de fermions em tres dimensões é realizado de maneira análoga ao caso de uma dimensão.

Supondo distribuição contínua de momenta, e usando novamente as Eqs. (A.11) e (A.12), temos, para a parte real de $\chi^{\circ}(\vec{q}, \omega)$ : 
102

$$
\operatorname{Re} \chi^{0}(\vec{q}, \omega)=\frac{3 \rho}{8 \pi \epsilon_{F}} \int_{\substack{|\vec{p}| \leq q_{F}\\}} d \vec{p}\left[\frac{1}{\omega-q^{2}+2 \vec{q} \cdot \vec{p}}-\frac{1}{\omega+q^{2}-2 \vec{q} \cdot \vec{p}}\right] .
$$

Efetuando a integração dentro dasuperfície de Fermi, chega-se a

$$
\begin{gathered}
\operatorname{Re} X^{0}(\vec{q}, w)=-\frac{3 \rho}{4 \epsilon_{F} q}\left\{2 q+\left[1-\left(\frac{w+q}{2 q}\right)^{2}\right] \log \left|\frac{w+\omega_{+}(q)}{w+\omega_{-}(q)}\right|+\right. \\
\left.+\left[1-\left(\frac{\omega-q^{2}}{2 q}\right)\right] \log \left|\frac{w-\omega_{+}(q)}{\omega-\omega_{-}(q)}\right|\right\},
\end{gathered}
$$

com $\omega_{ \pm}(q)=q^{2} \pm 2 q$.

Para a parte imaginária, usando as Eqs. (A.9) e (A.12), e supondo apenas frequências $\omega>0$, obtémse

$$
I_{m} \chi^{0}(\vec{q}, \omega)=-\frac{3 \rho}{8 \epsilon_{F}} \int d \vec{p} \eta_{\vec{p}}^{0}\left(1-\eta_{\vec{p}+\vec{q}}^{0}\right) \delta\left(\omega-\omega_{\vec{p} \vec{q}}\right) .
$$

Para que a integral não seja nula, devemos ter $|\vec{p}|<q_{F}<|\vec{p}+\vec{q}|$, is to $\bar{e}$, se $\theta \bar{e}$ o angulo entre $\vec{p}$ e $\vec{q}$, devemos ter

$$
|\vec{p}| \leq q_{F}
$$

e $\quad \cos \theta \geqslant \alpha(p, q)=\frac{q^{2}-q^{2}-p^{2}}{2 q p}$.

Assim, a Eq. (A.19) fica

$$
I_{m} X^{0}(\bar{q}, \omega)=\frac{3 \pi \rho}{8 q p \epsilon_{F}} \int_{0}^{q_{F}} d p p^{2} \int_{\alpha(q, p)}^{1} d x \delta\left(x-\frac{\omega-q^{2}}{2 q p}\right) \text {. }
$$


103

Resolvendo a integral da equação (A.21), com a condição (A.20), chega-se a

$$
\begin{array}{rlrl}
I_{m} X^{0}(q, \omega) & =-\frac{3 \pi \rho}{8 \epsilon_{F}} \frac{\omega}{q} & 0 \leq \omega \leq|\omega-(q)| \\
& =-\frac{3 \pi \rho}{8 \epsilon_{F} q}\left[1-\left(\frac{\omega-q^{2}}{2 q}\right)\right] & & \left|\omega_{-}(q)\right| \leq \omega \leq \omega_{+}(q) \\
& =0 & \omega_{+}(q)<\omega
\end{array}
$$

$(A, 22)$

A susceptibilidade generalizada de um gás de fermions tridimensiona1, sem interação, é então expressa pelas equações (A.18) e (A.22) .

A. 1 - SISTEMAS DE BOSONS

Em um sistema de bosons sem interação, todas as partículas podem se encontrar no mesmo estado de partícula única, com momentum zero, correspondendo ao estado fundamental do sistema total. Então, ao calcular os elementos de matriz do operador densidade que aparecem na Eq. (A.1), vamos acoplar estados com momentum zero ao estado com momentum $\vec{q}$, que é o momen tum resultante do par partícula-buraco que criamos. Assim, neste caso vamos ter $\hbar \omega_{\text {no }}=\varepsilon_{q}=\hbar^{2} q^{2} / 2 m$. A expressão para a susceptibilidade pode ser escrita como (em unidades adimensionais):

$$
\chi^{0}(\vec{q}, \omega)=\frac{1}{\epsilon_{F}} \sum_{n}\left|\left(\rho_{\bar{q}}\right)_{n 0}\right|^{2} \frac{2 \epsilon_{\bar{q}}}{\left(\bar{\hbar} \omega^{*}\right)^{2}-\left(\epsilon_{\bar{q}}\right)^{2}}
$$


Porēm, neste caso, temos aind $\sum_{n}\left\langle 0\left|\rho_{\vec{q}}^{+}\right| n\right\rangle\left\langle n\left|\rho_{\vec{q}}\right| 0\right\rangle=\left\langle 0\left|\rho_{\vec{q}}^{+} \rho_{\vec{q}}\right| 0\right\rangle=\rho$ onde $\rho$ representa o número de partículas em um volume unitário. Então, a susceptibilidade do gās de bosons sem interação, vale

$$
\chi^{0}(\vec{q}, \omega)=\frac{2 \rho \in(\vec{q})}{(\omega+i \eta)^{2}-[\epsilon(\vec{q})]^{2}},
$$

que é a expressão vālida para sistemas com qualquer dimensionalidade. 


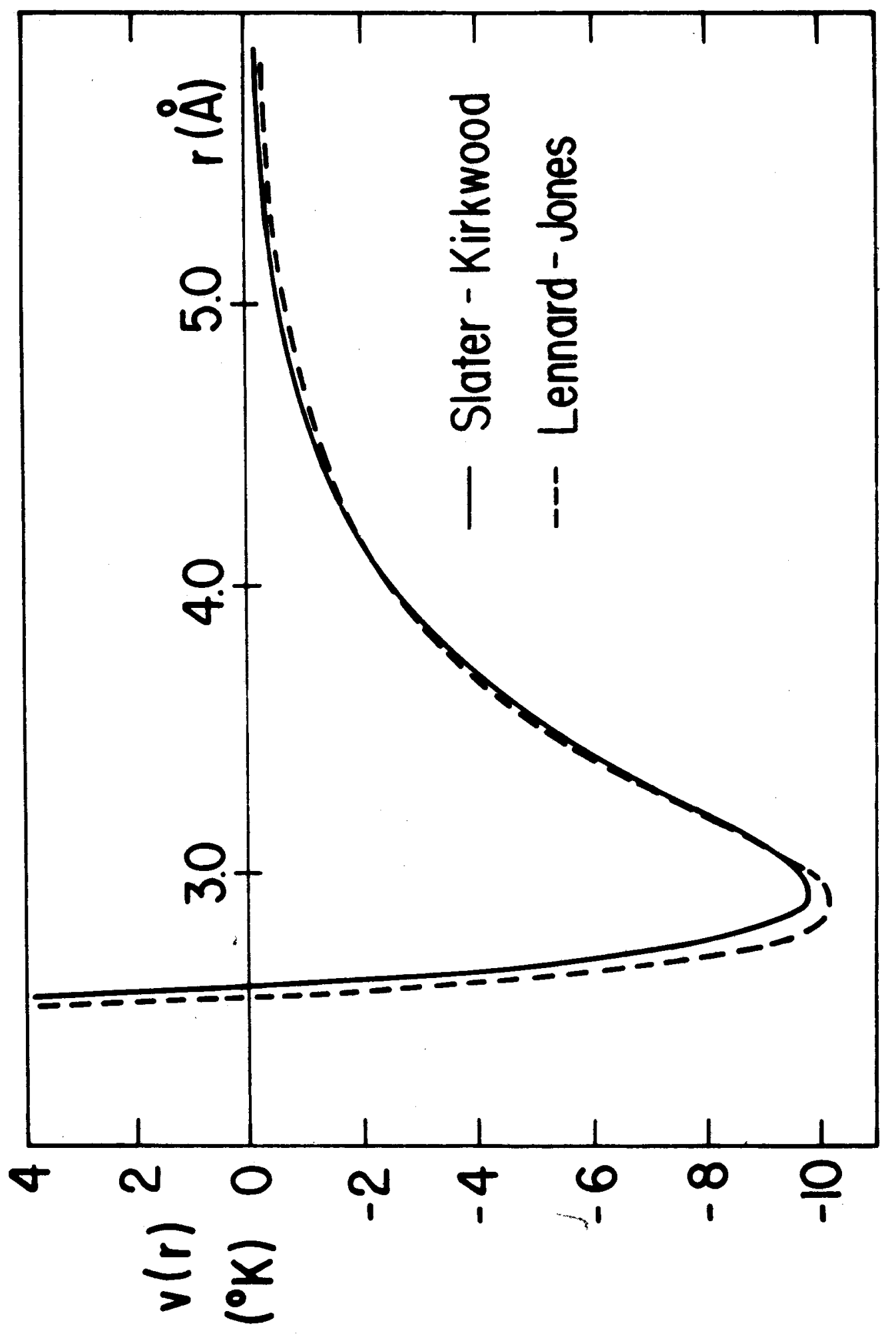

FIGURA 1 


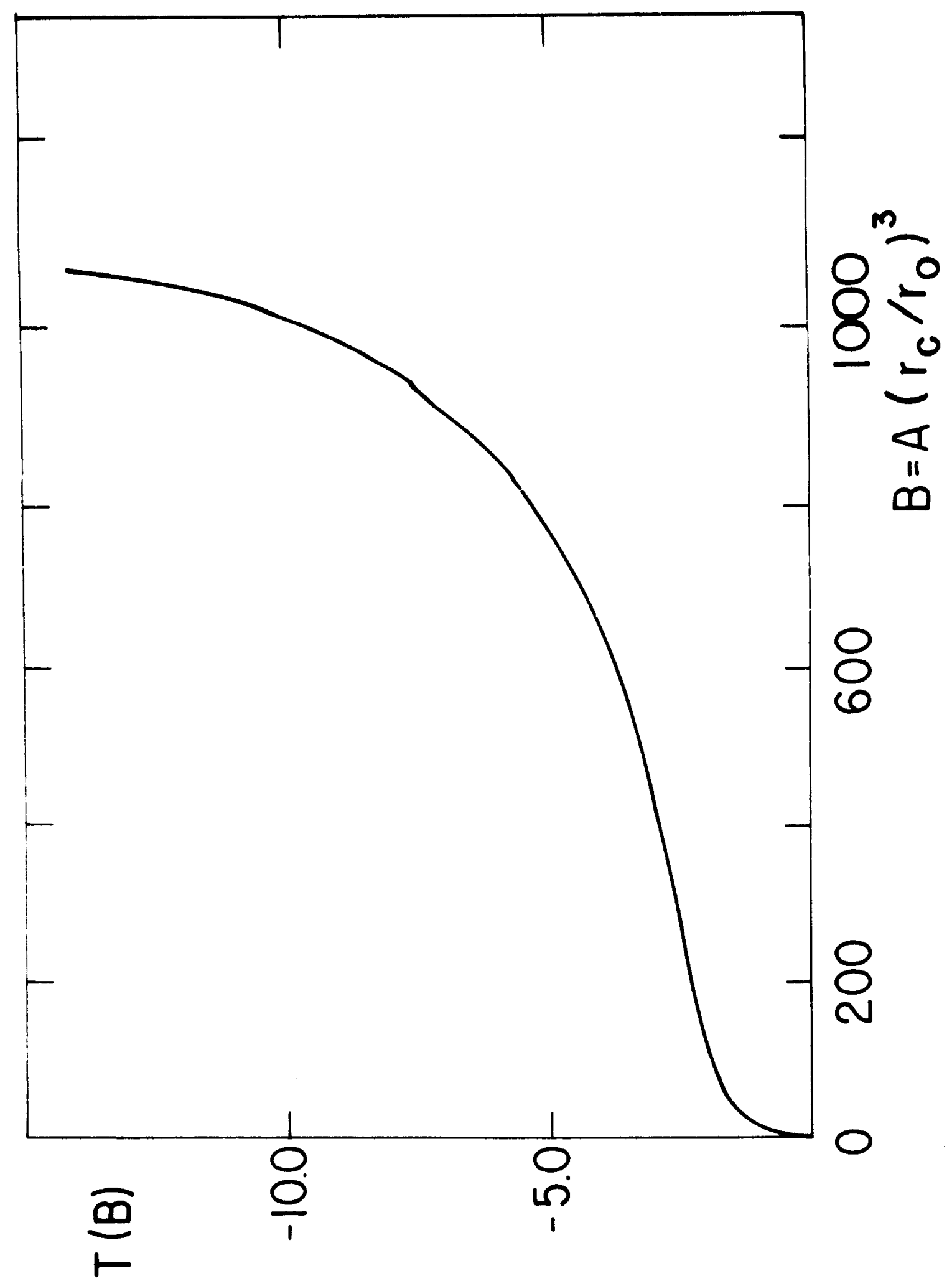

FIGURA 2 


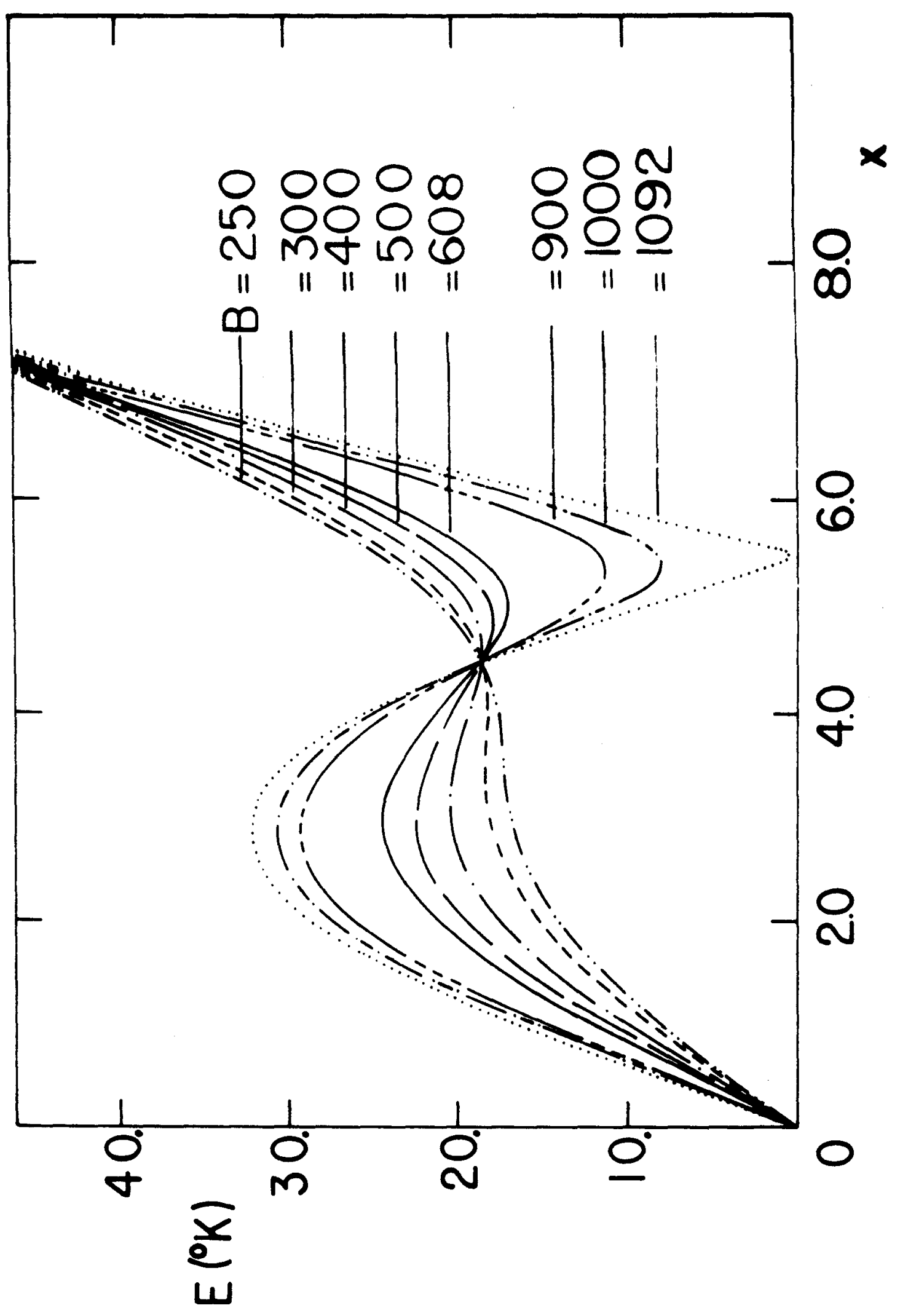

FIGURA 3 


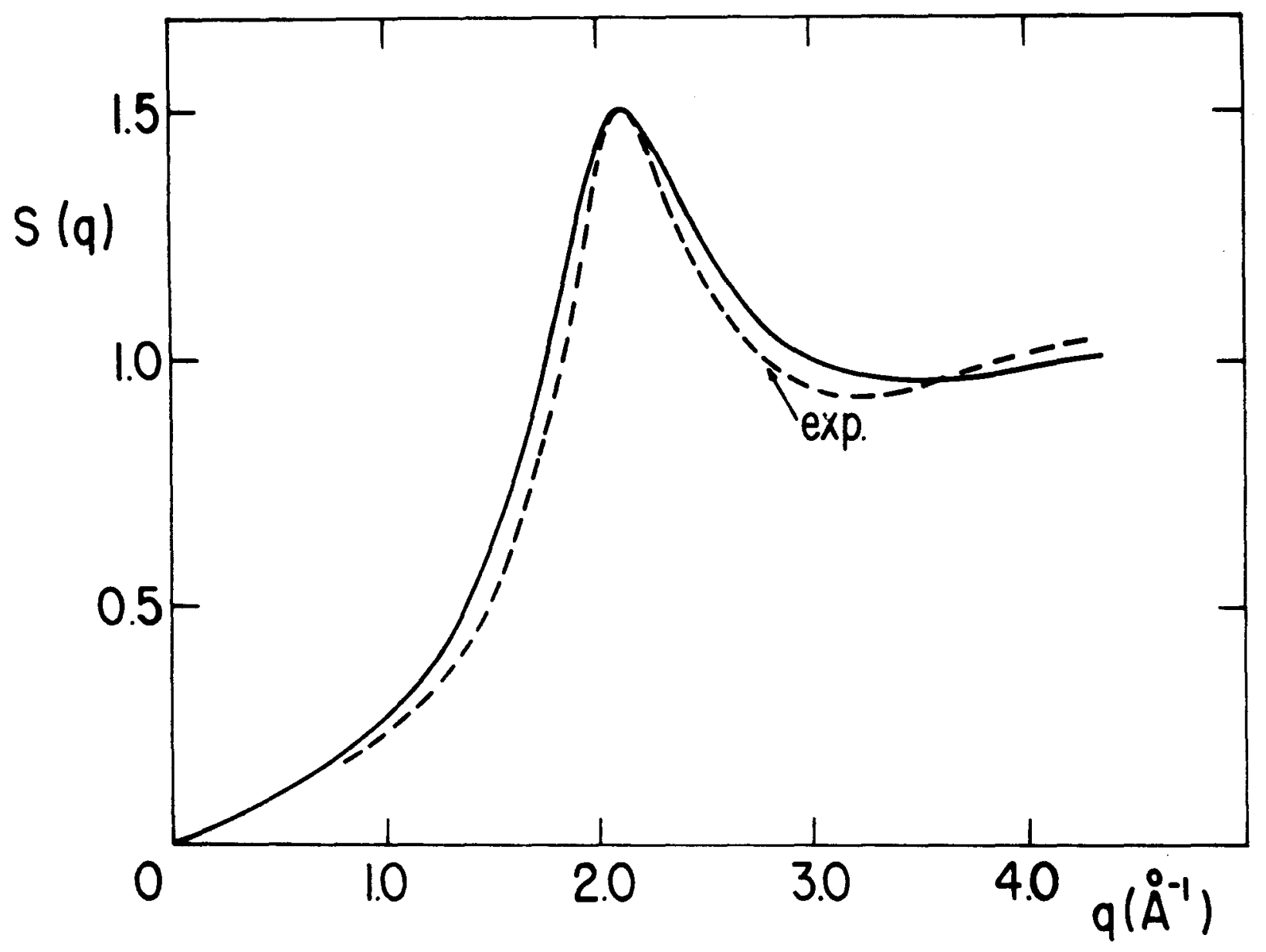

FIGURA 4 


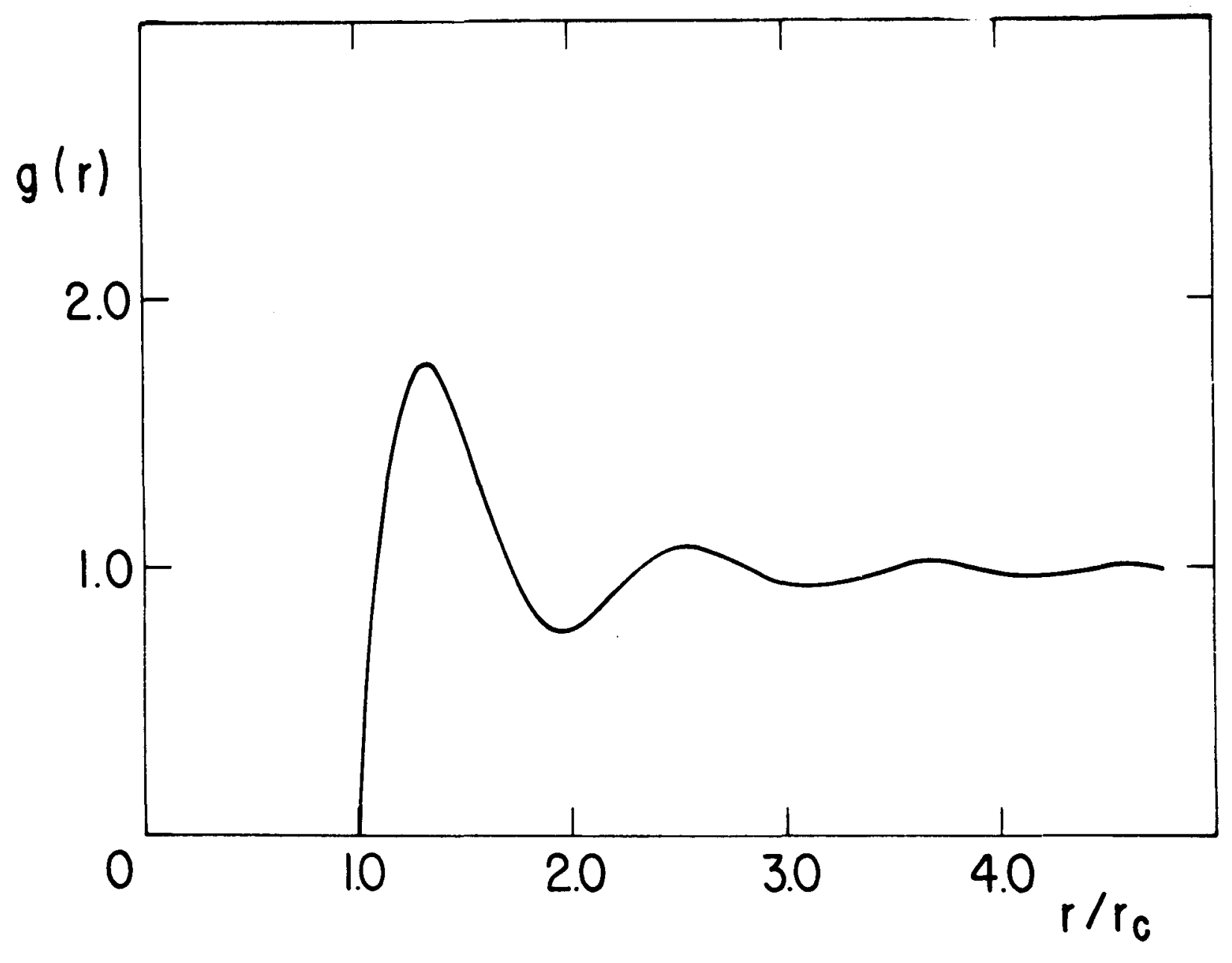

FIGURA 5 


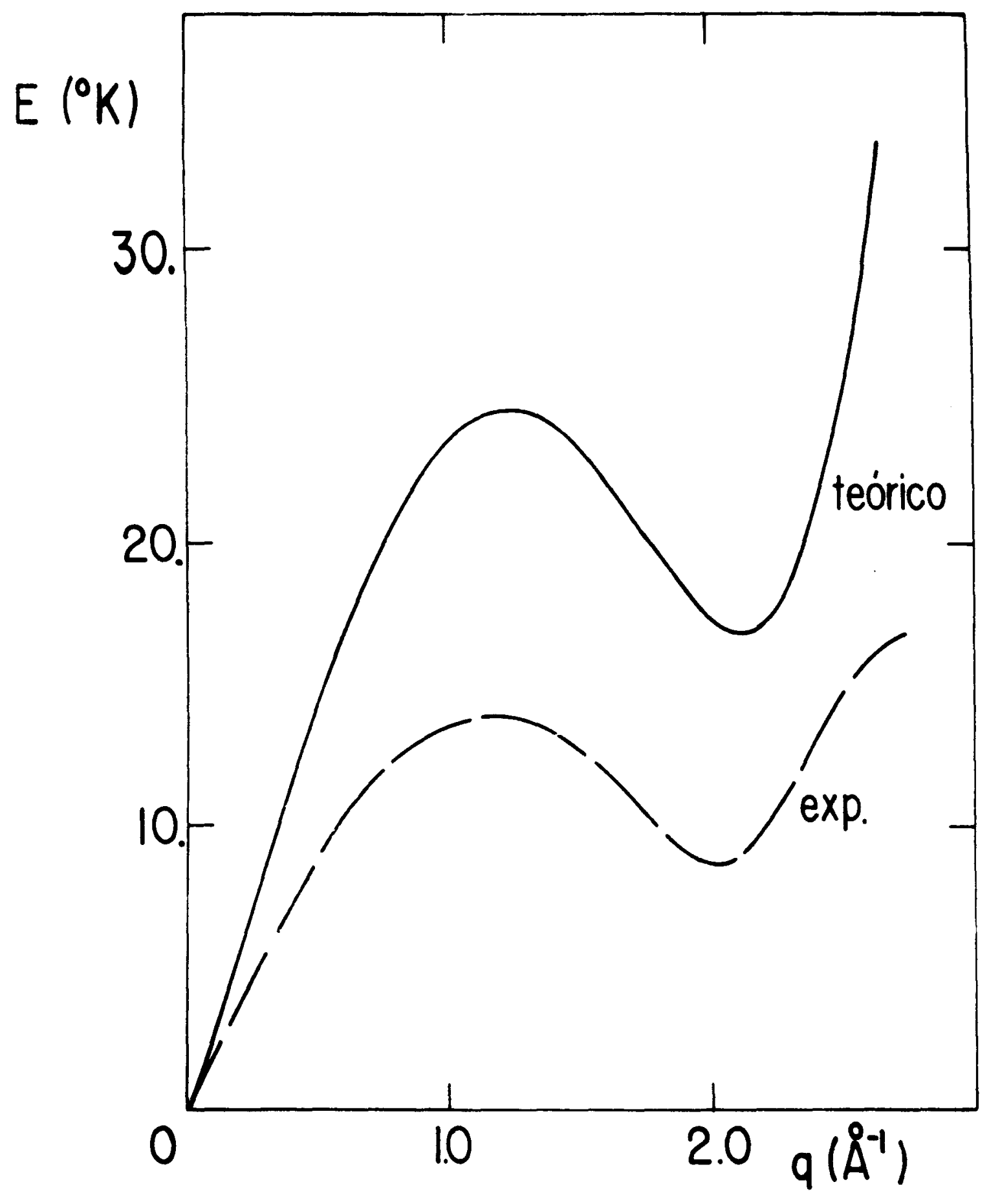

FIGURA 6 


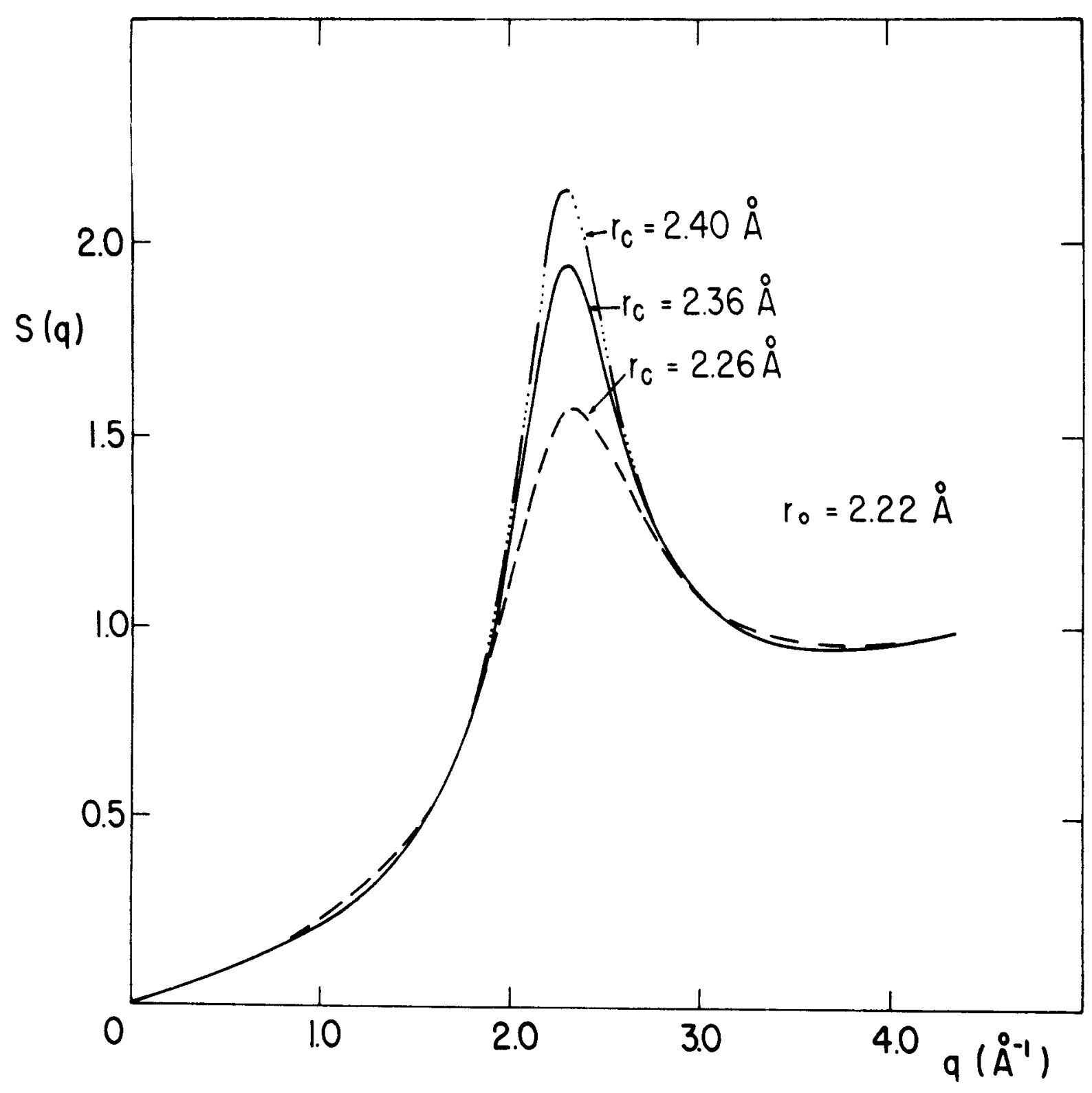

FIGURA 7 


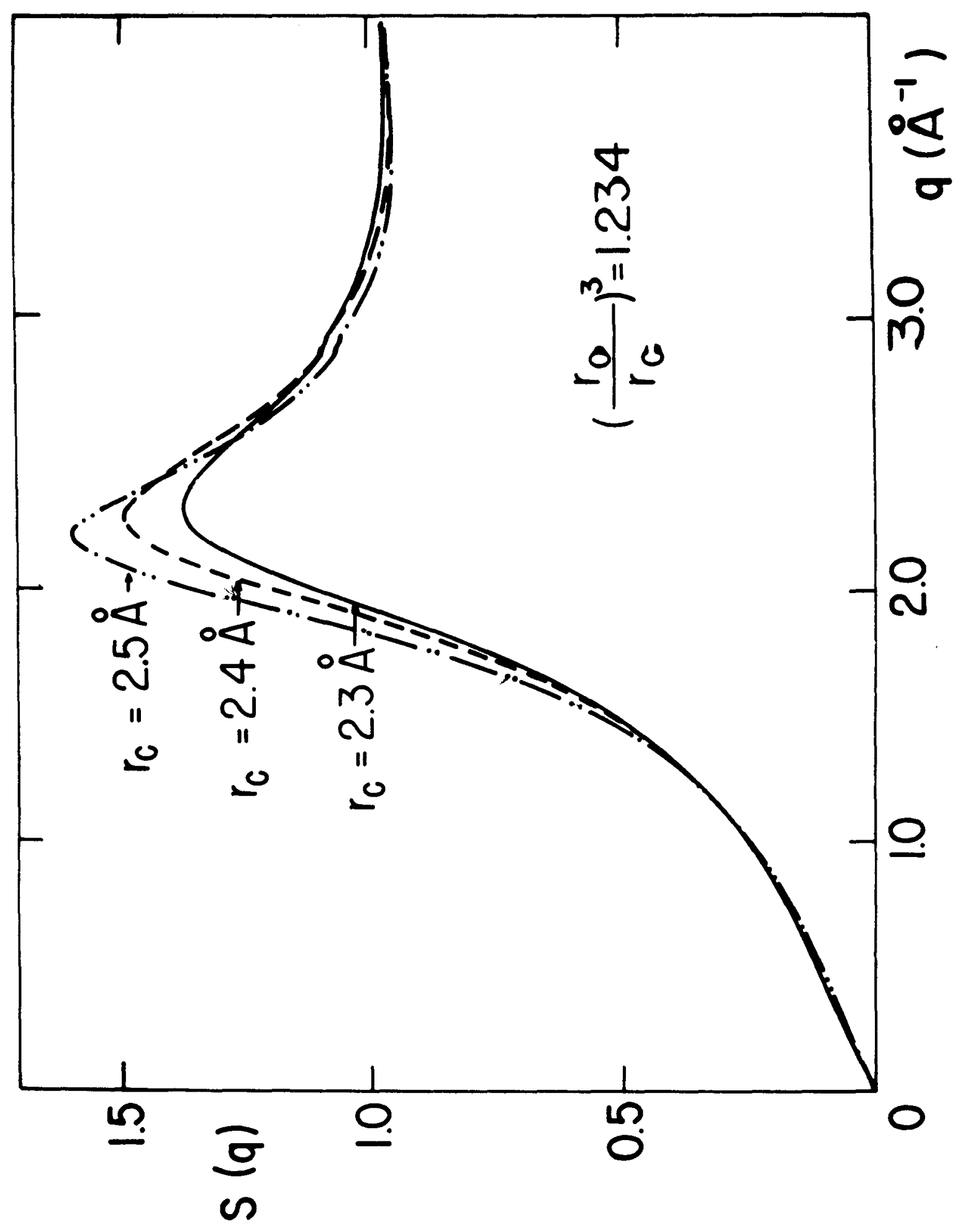

FIGURA 8 


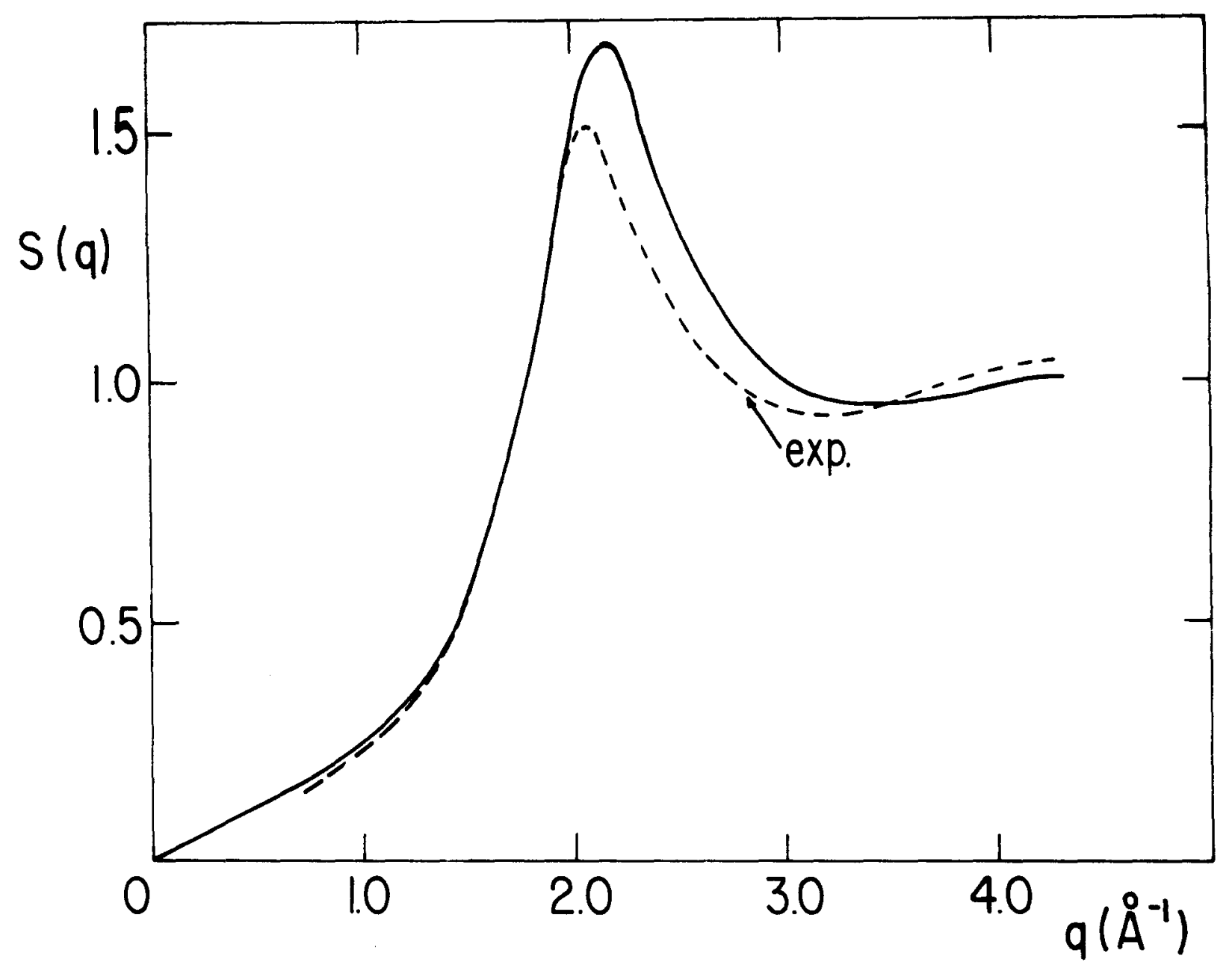

FIGURA 9 


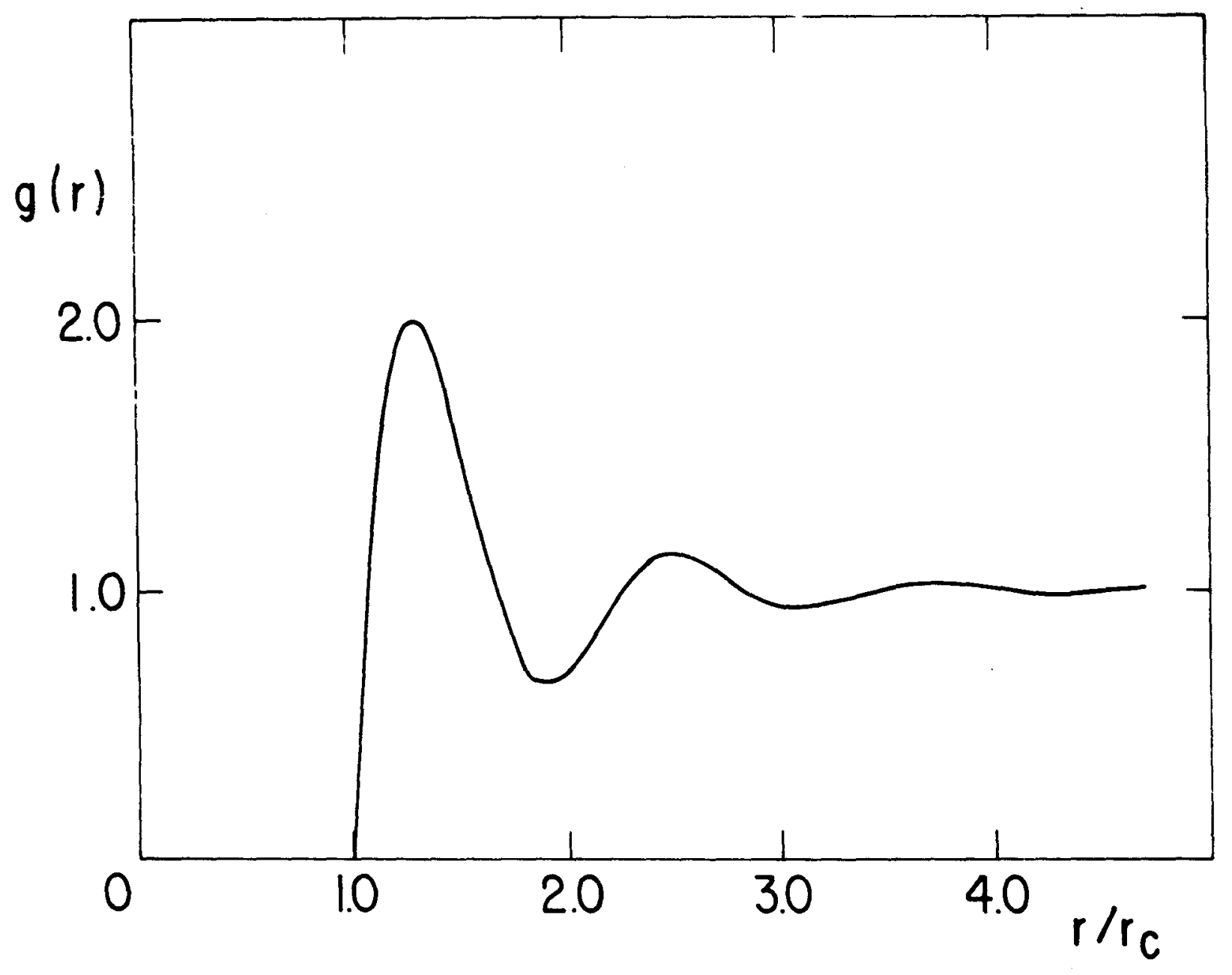

FIGURA 10 


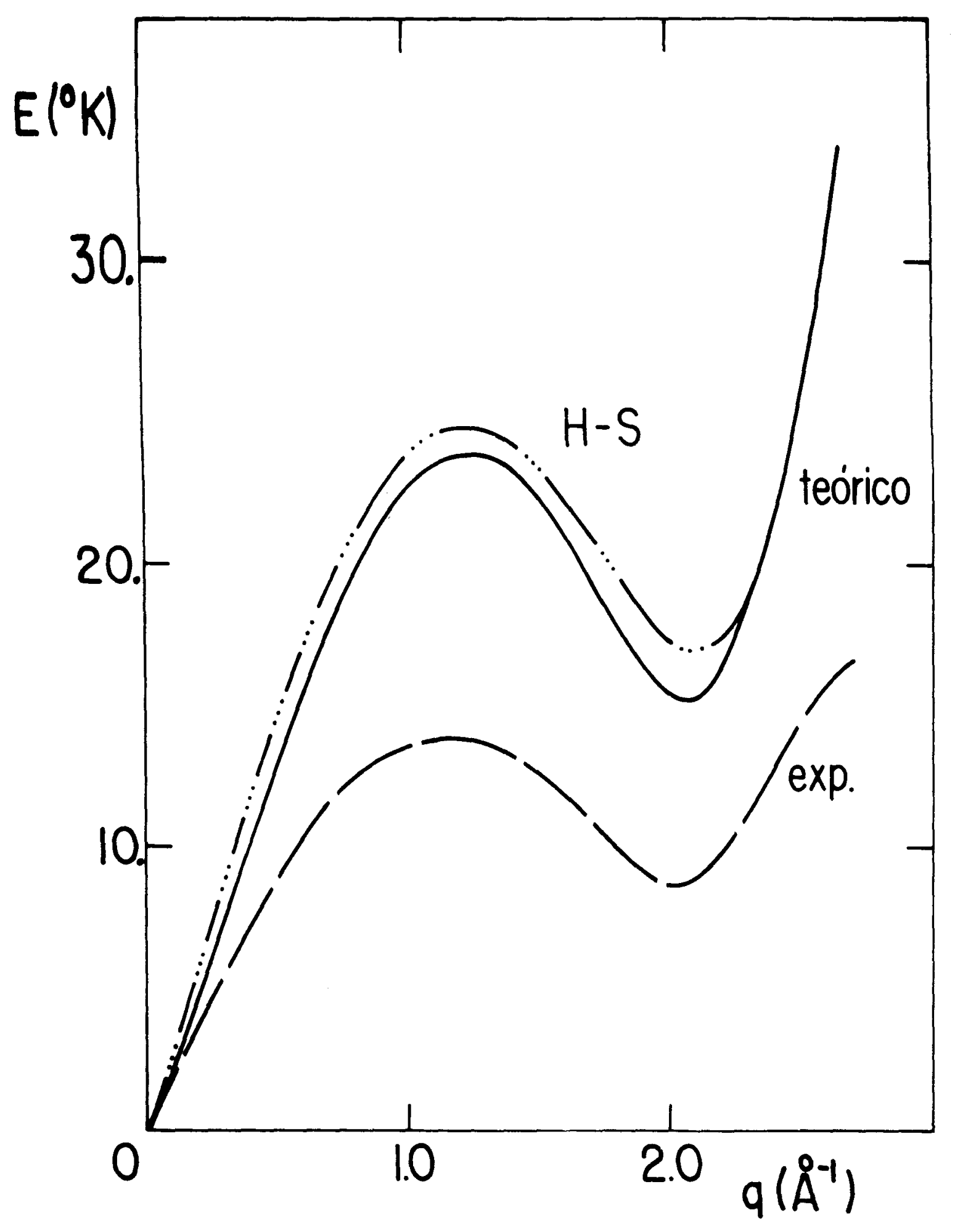

FIGURA 11 


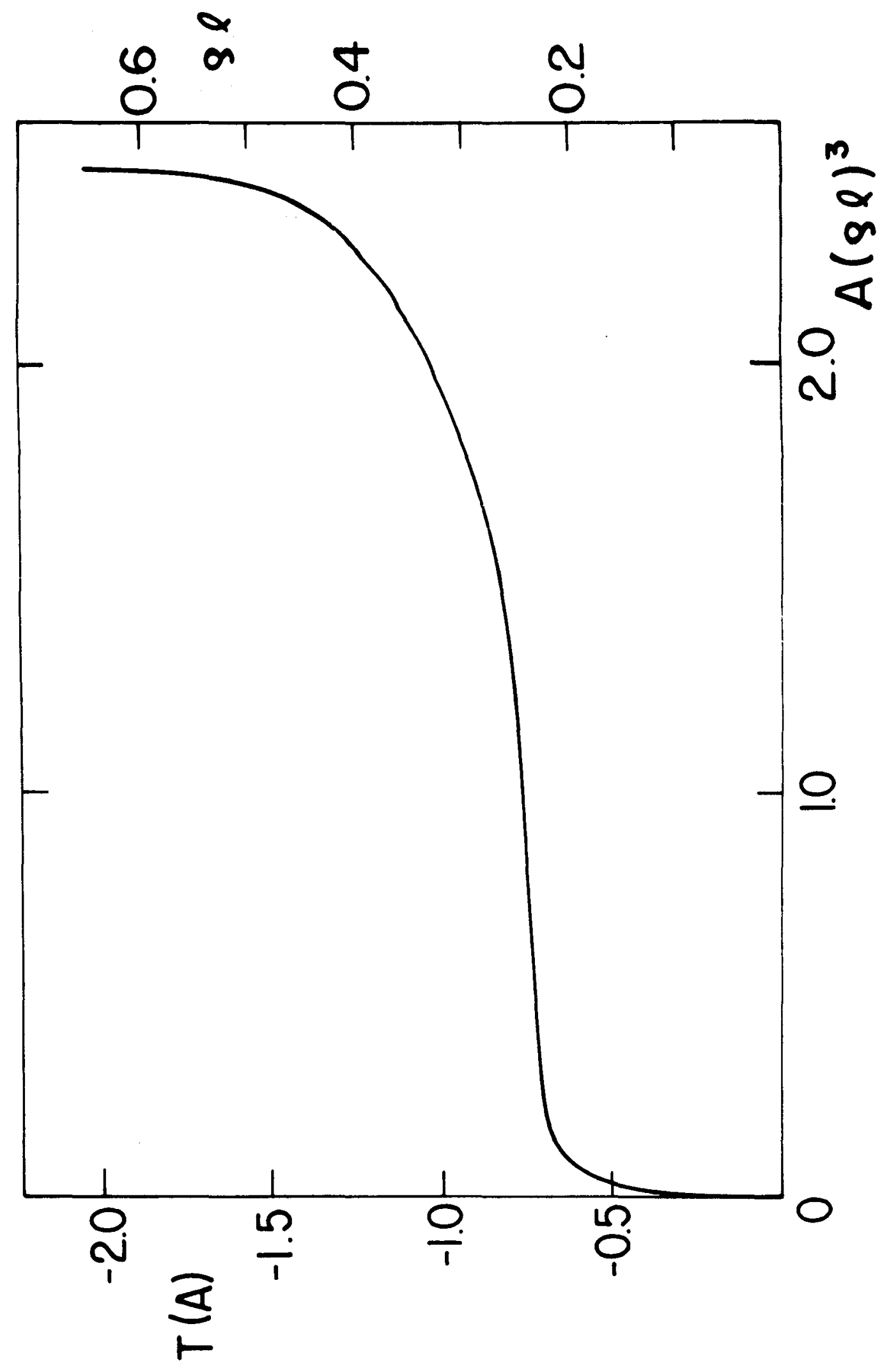

FIGURA 12 

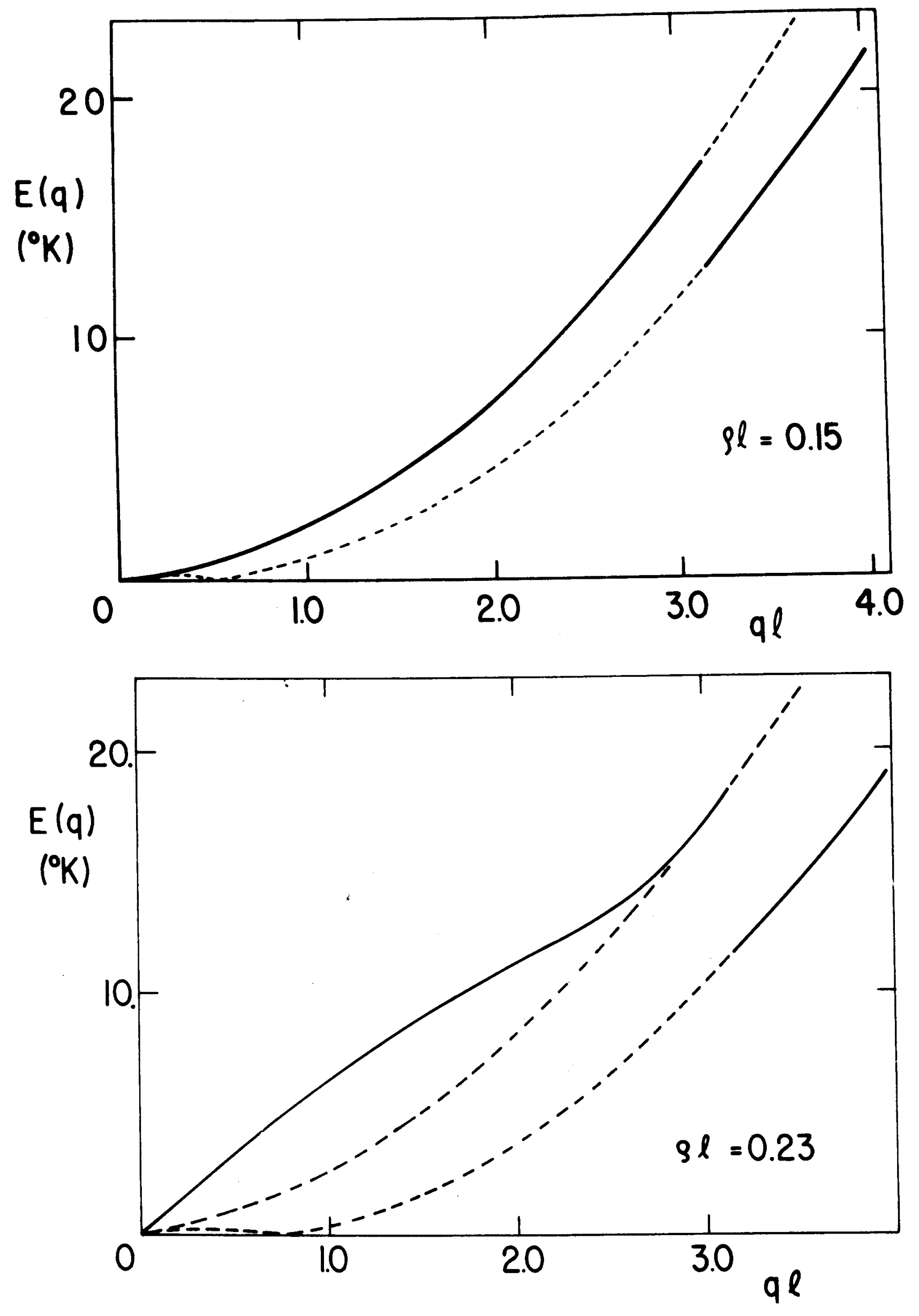

FIGURA 13 

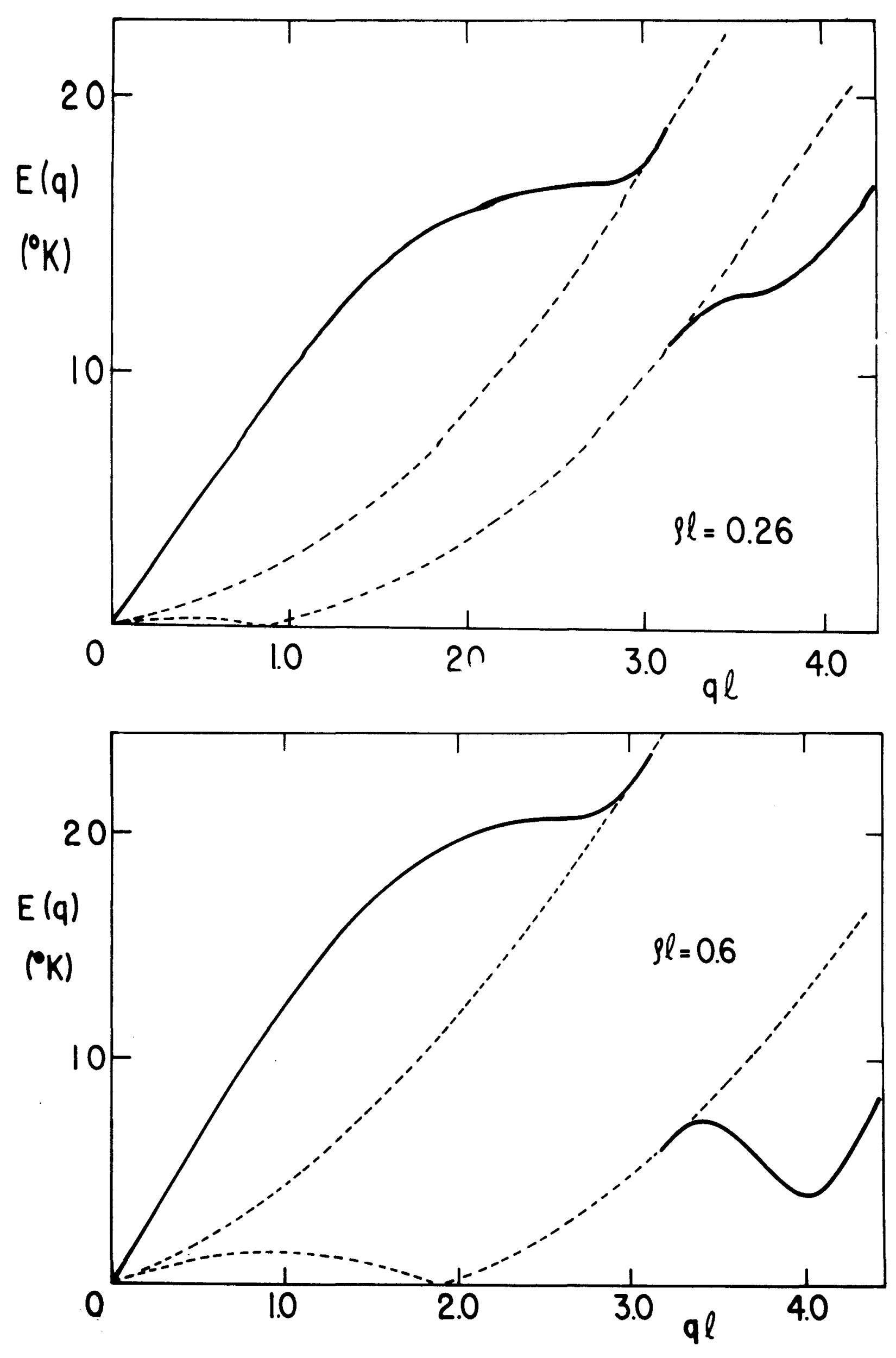

FIGURA 14 


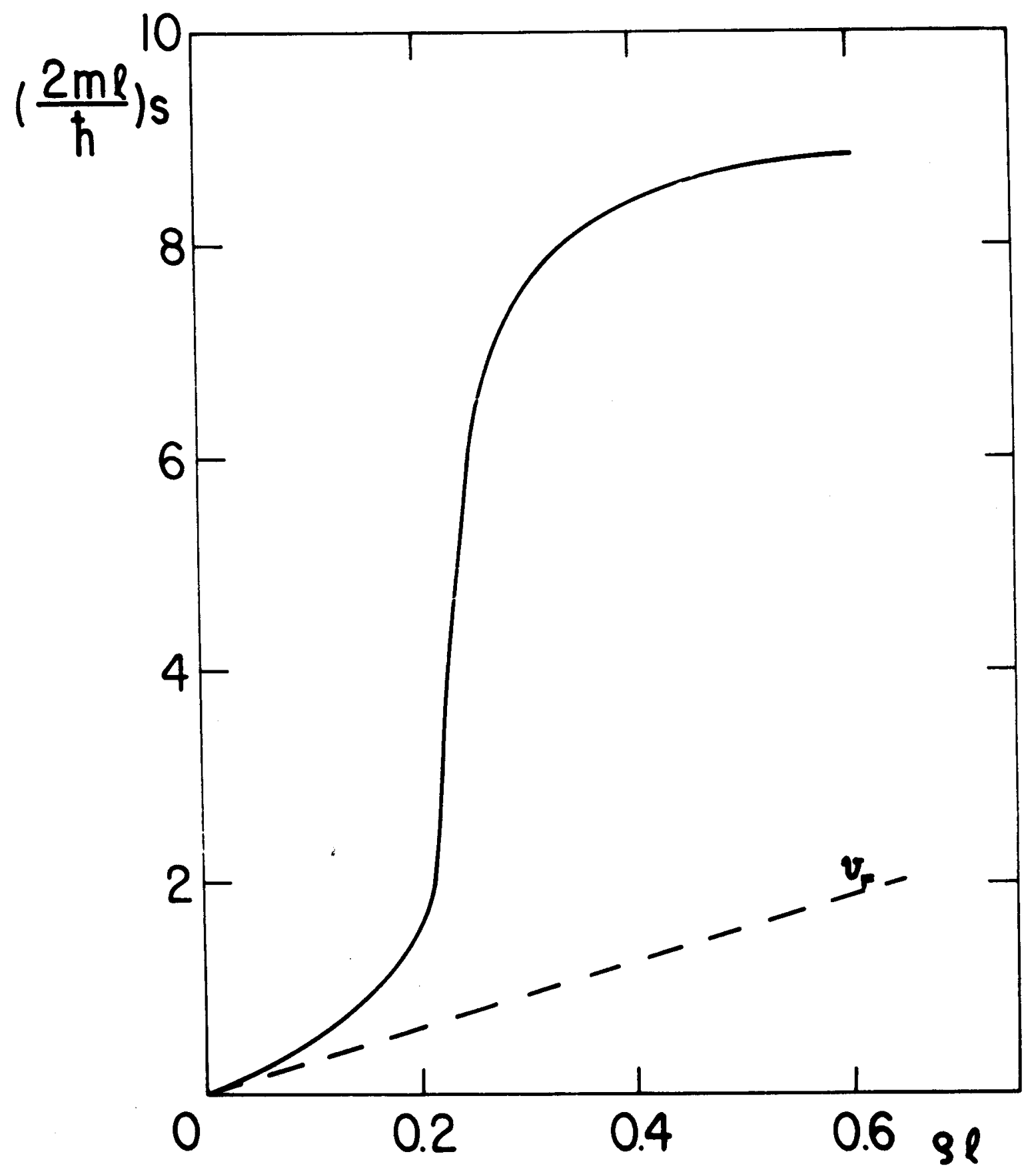

FIEURA 15 

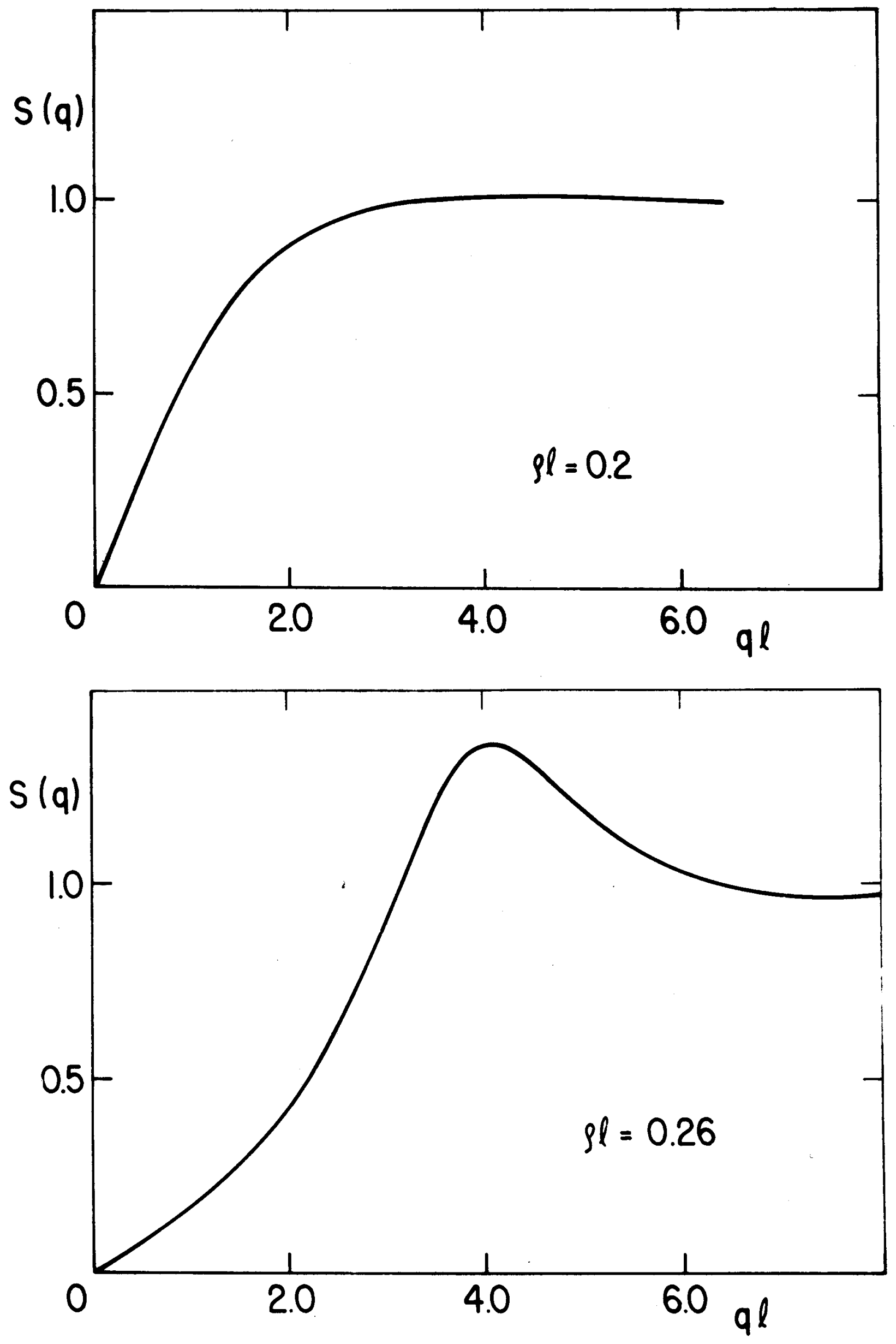

FIGURA 16 

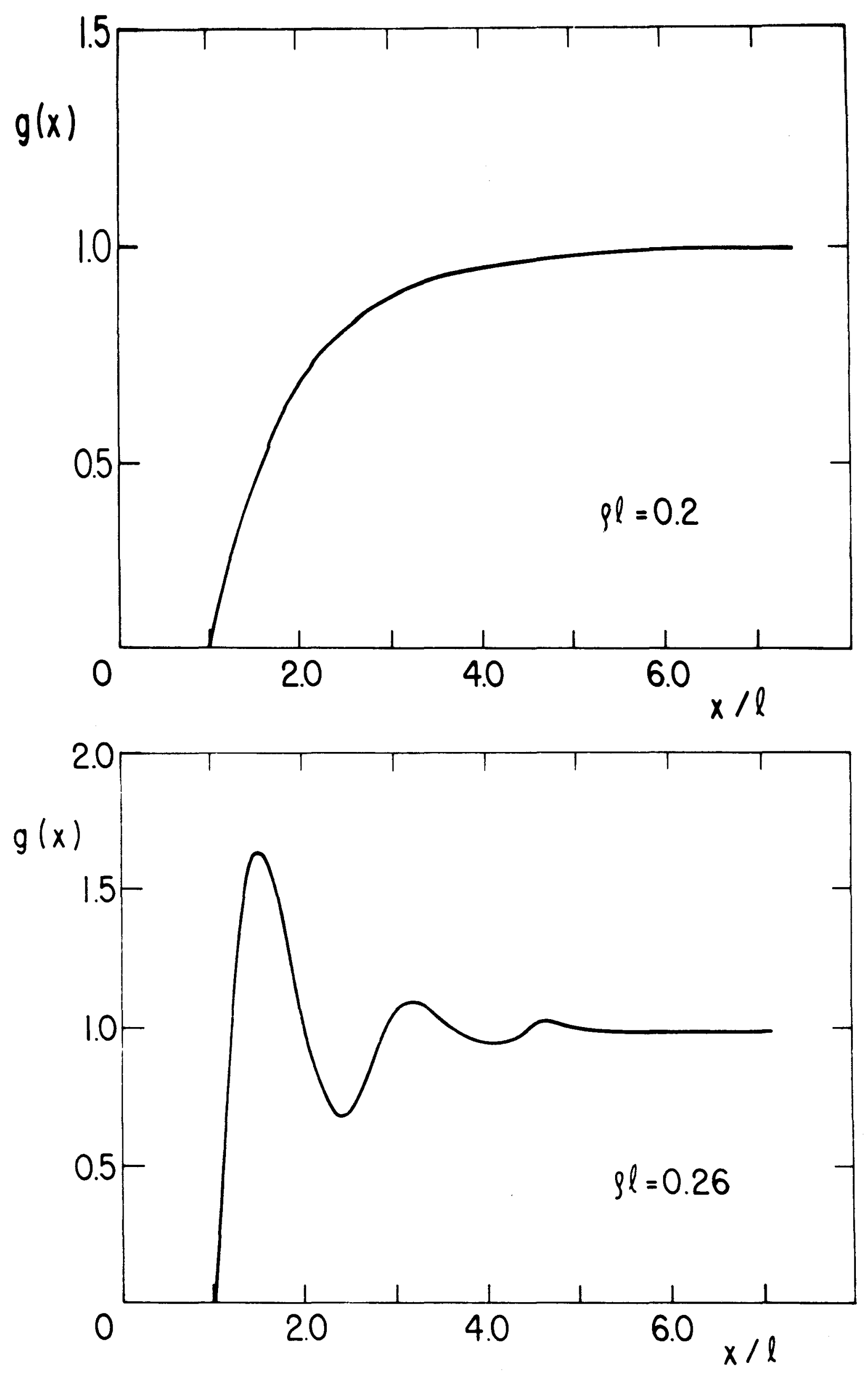

FIGURA 17 


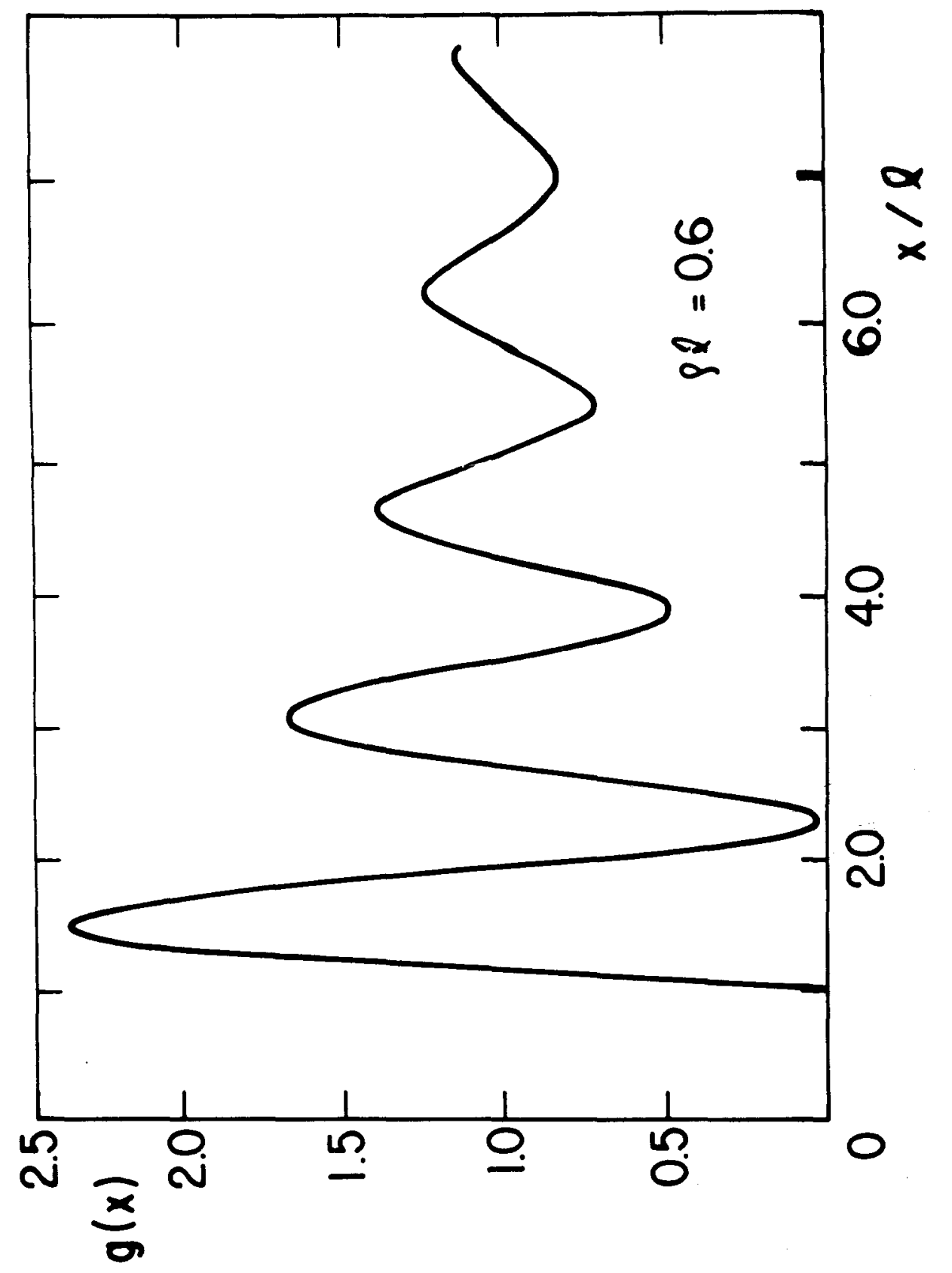

FIGURA 18 


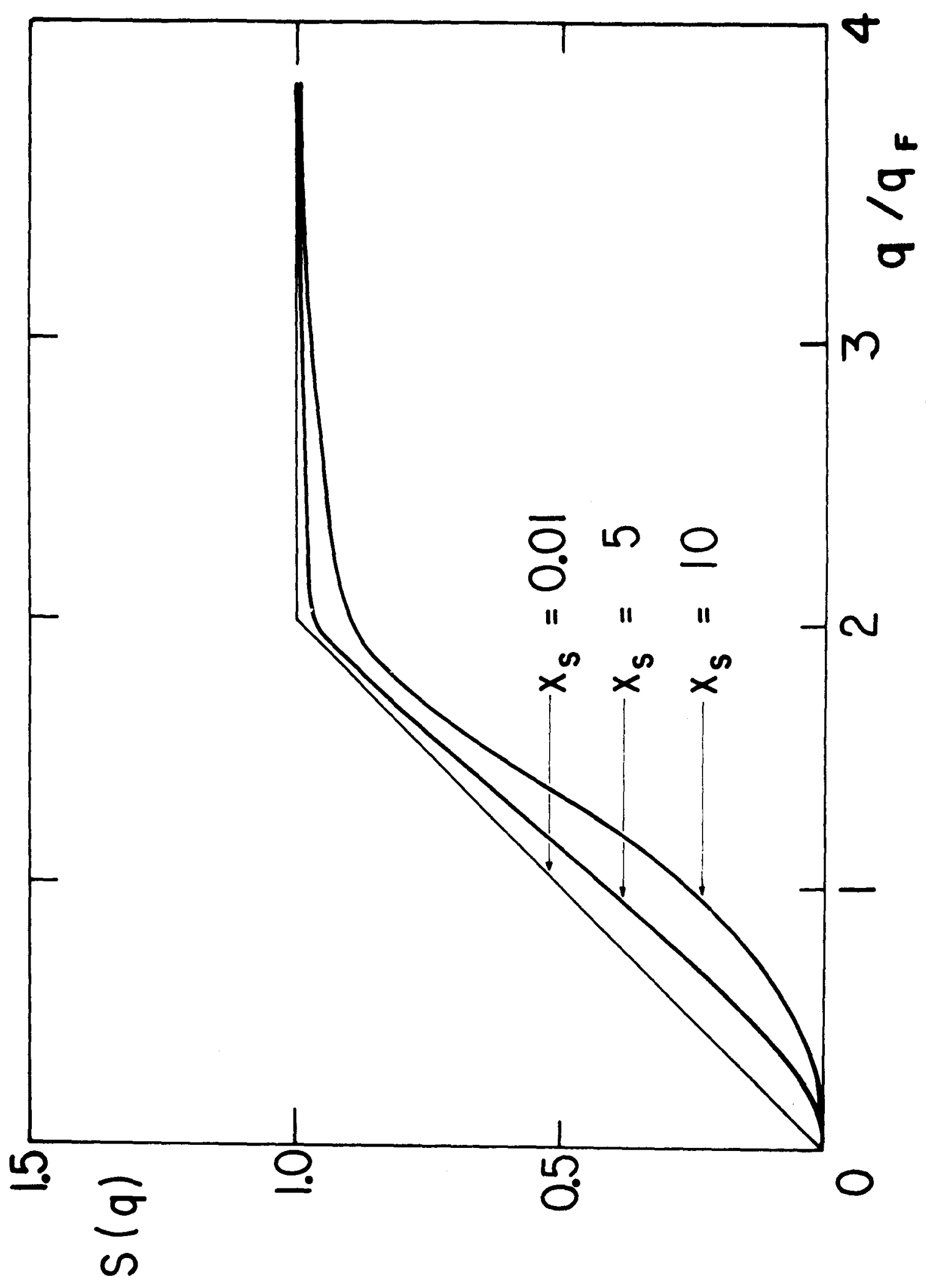

FIGURA 19 

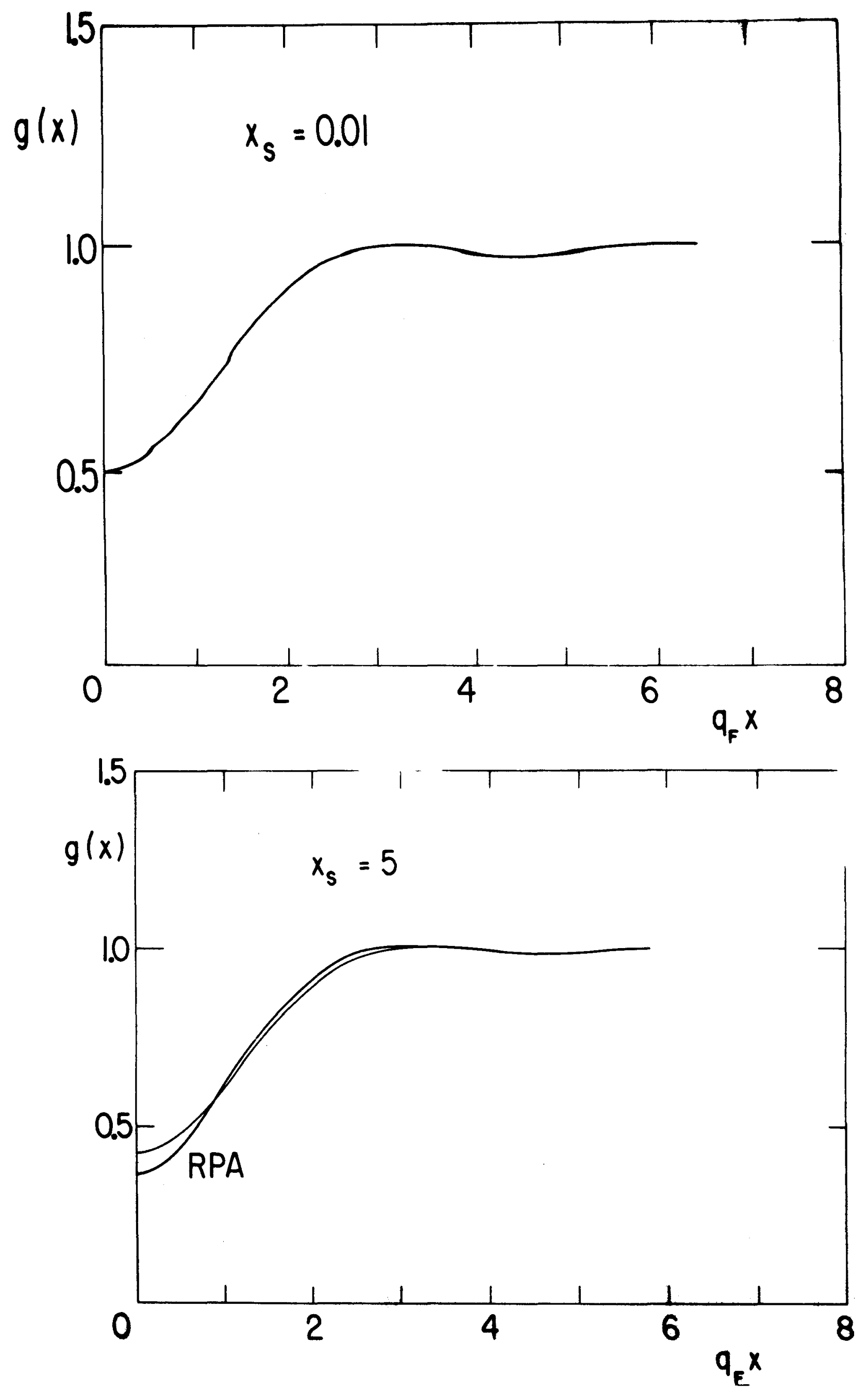

FIGURA 20 

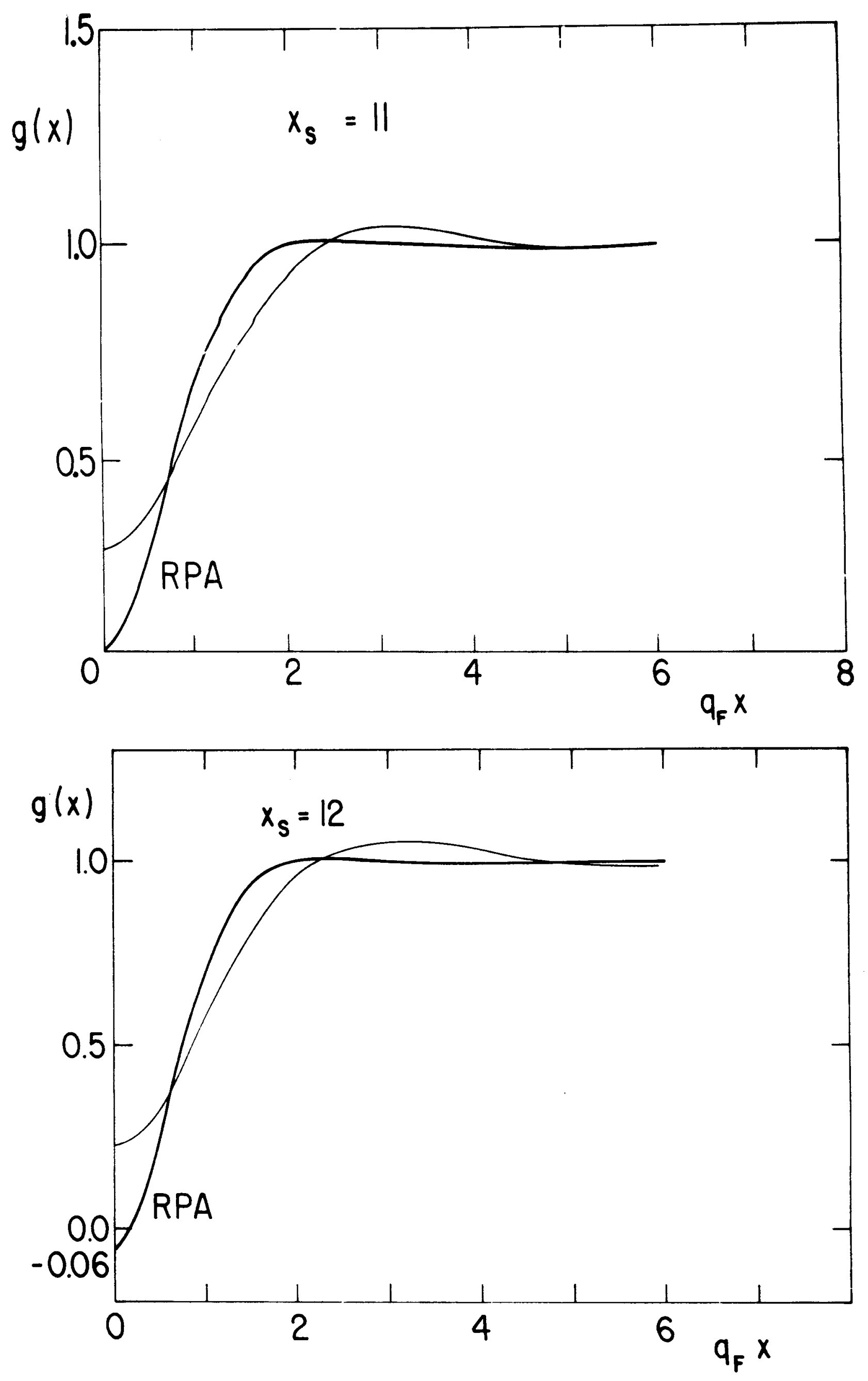

FIGURA 21 


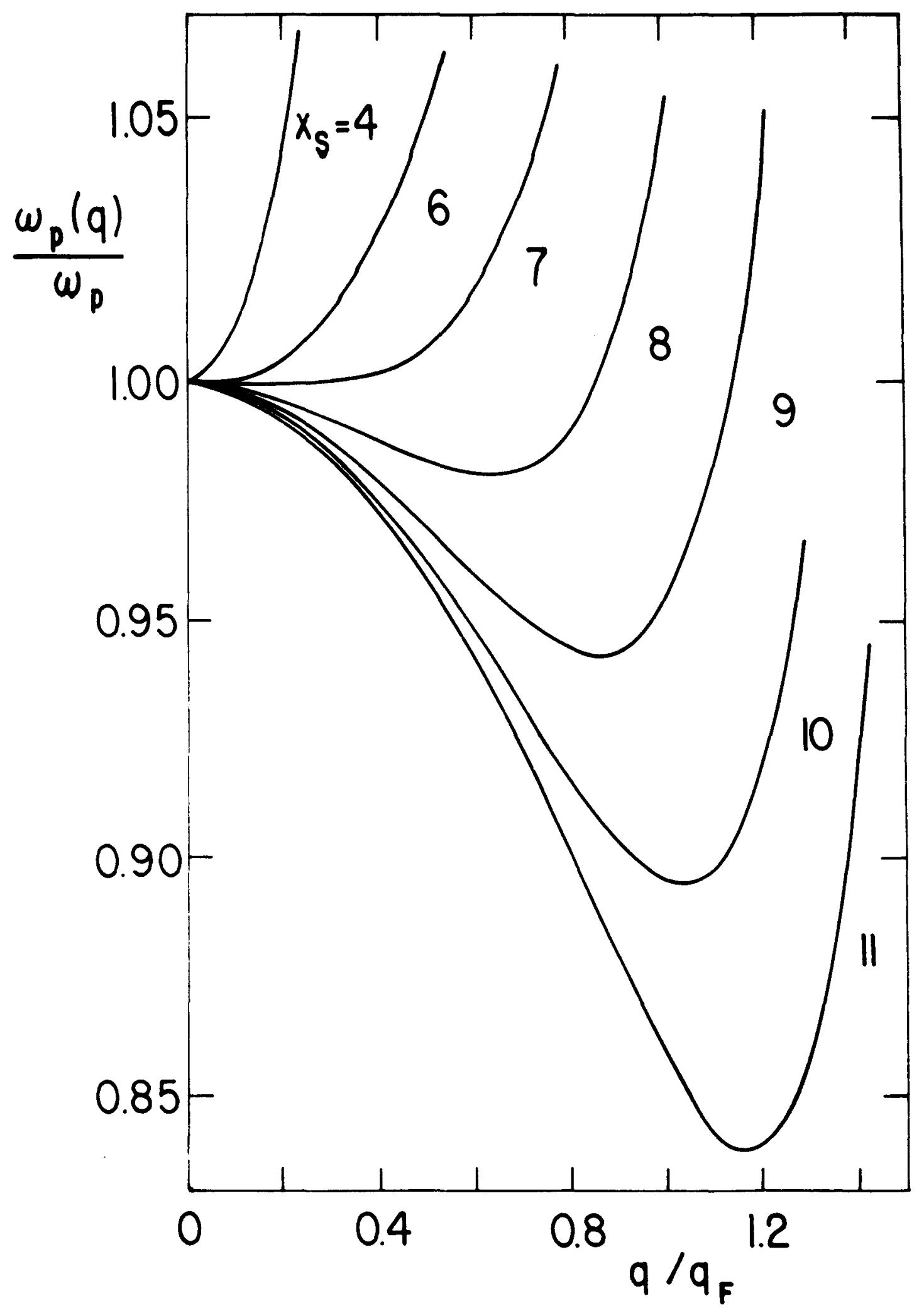

FIGURA 22 


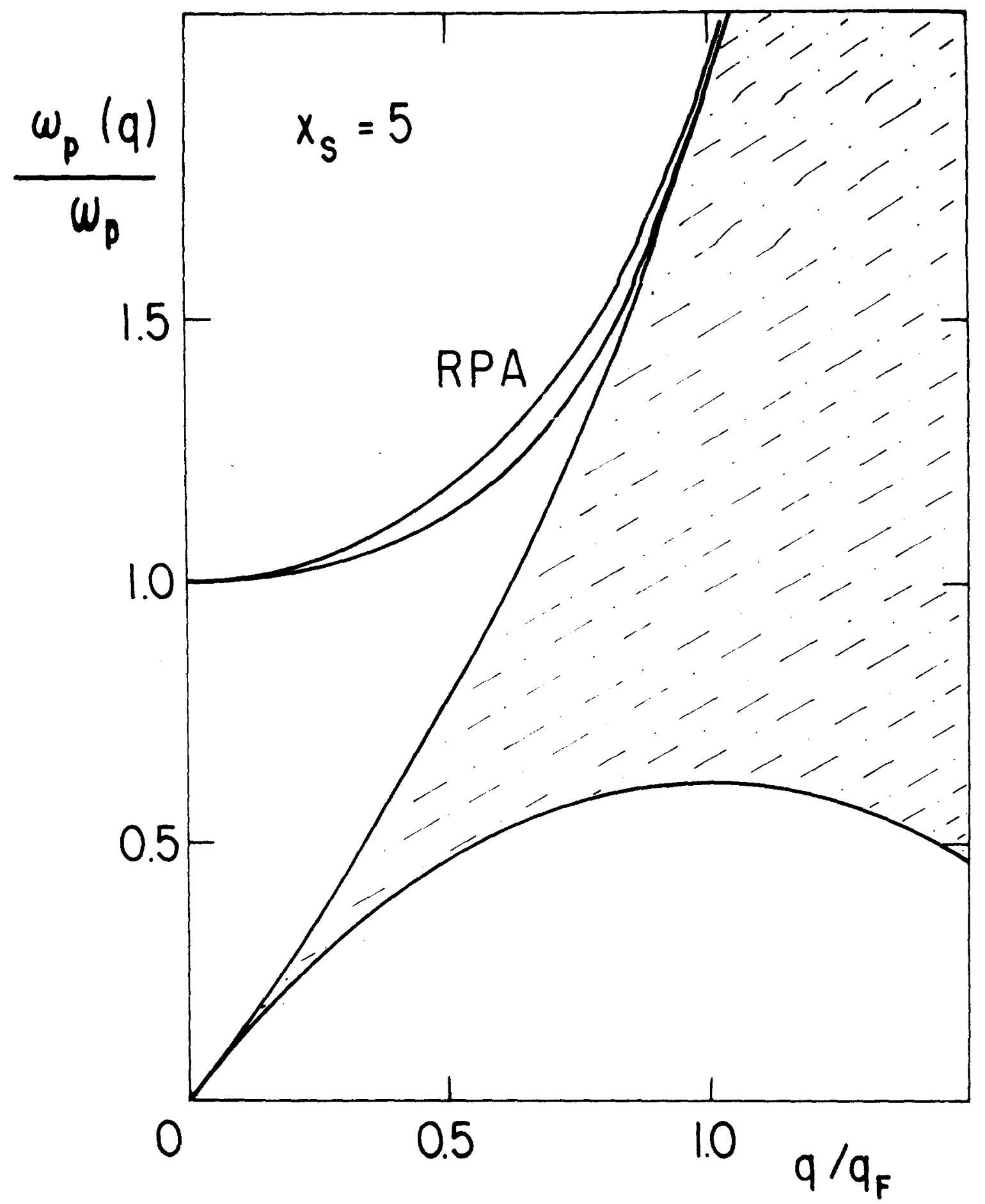

FIGURA 23 


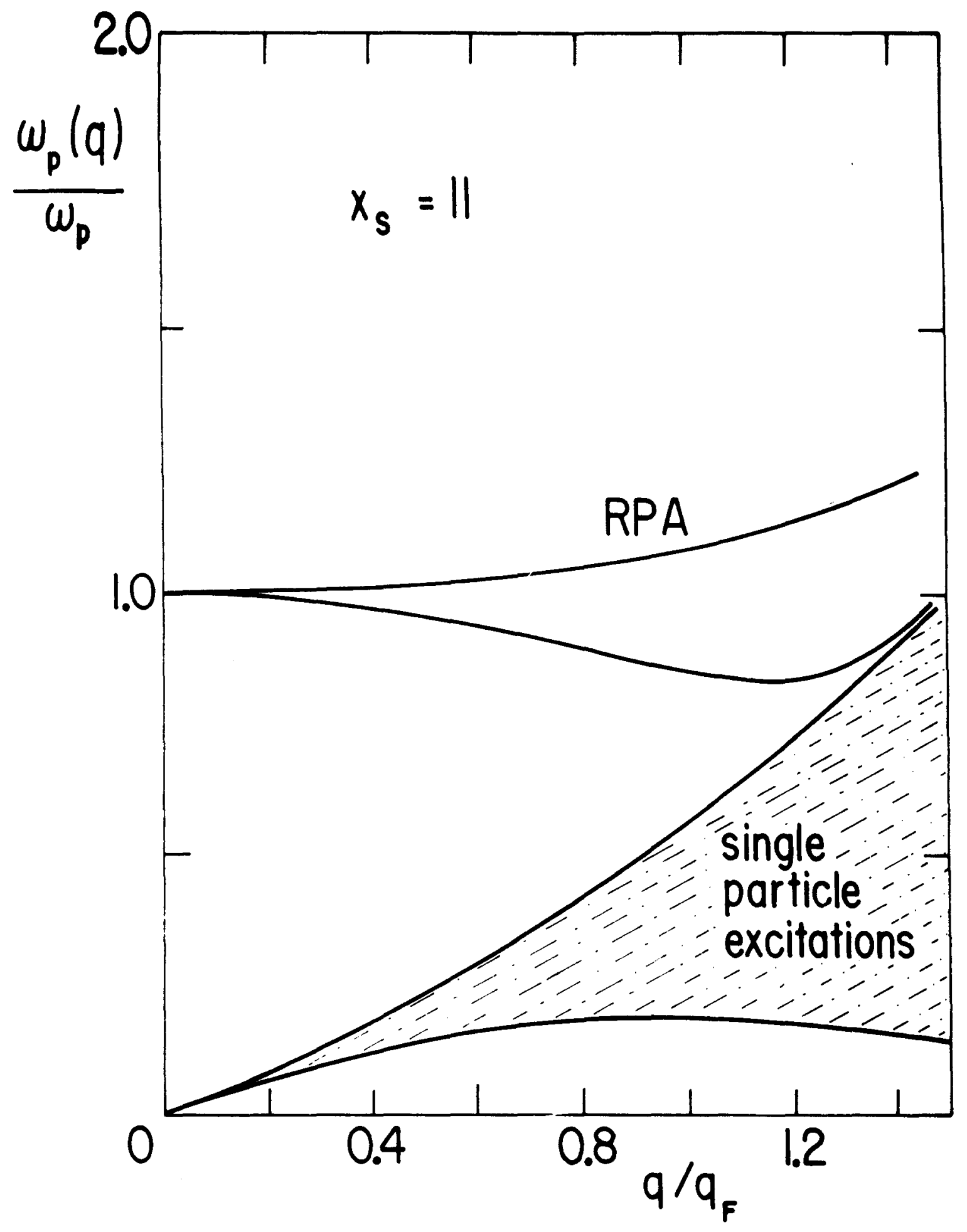

FIGURA 24 


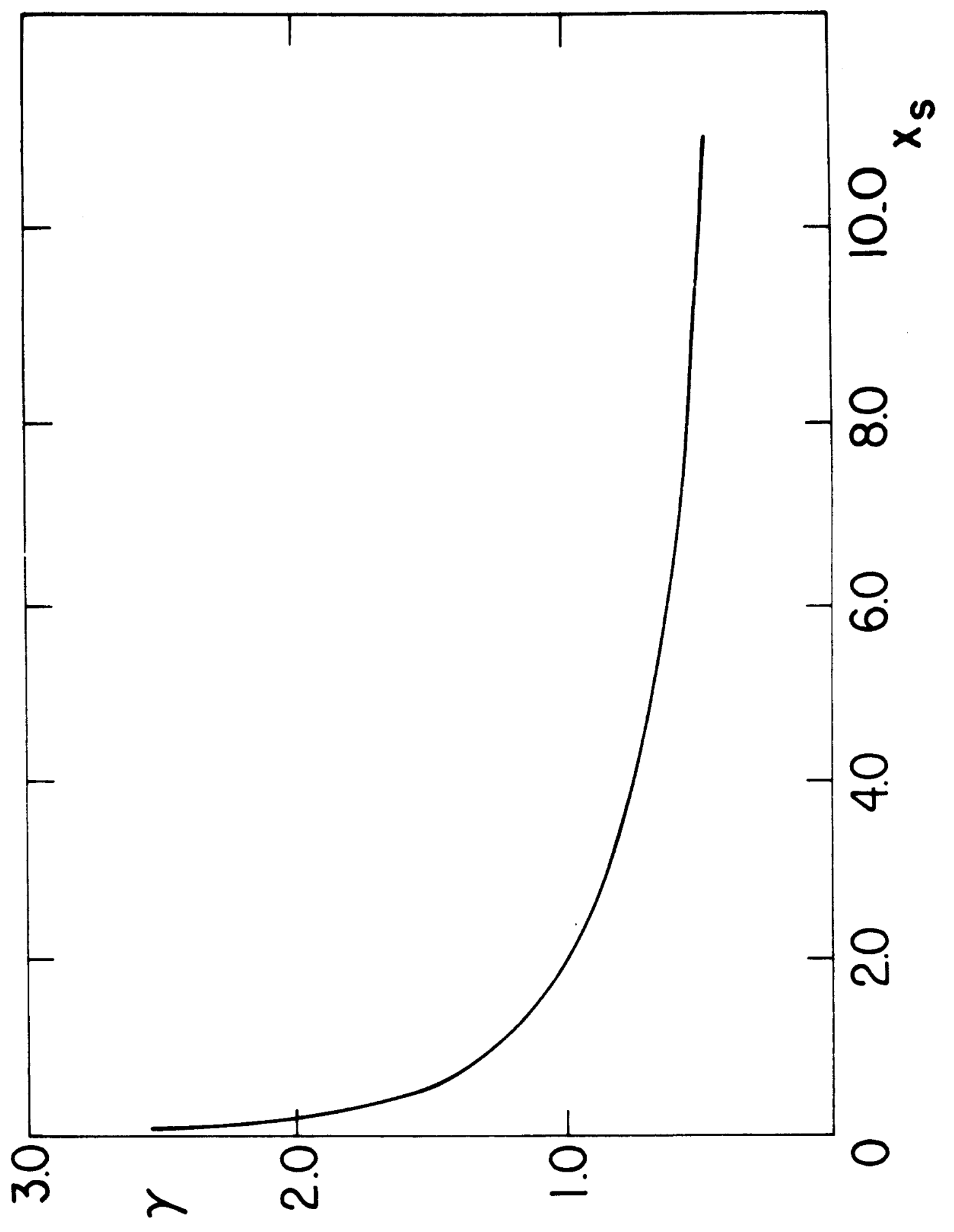

FIGURA 25 


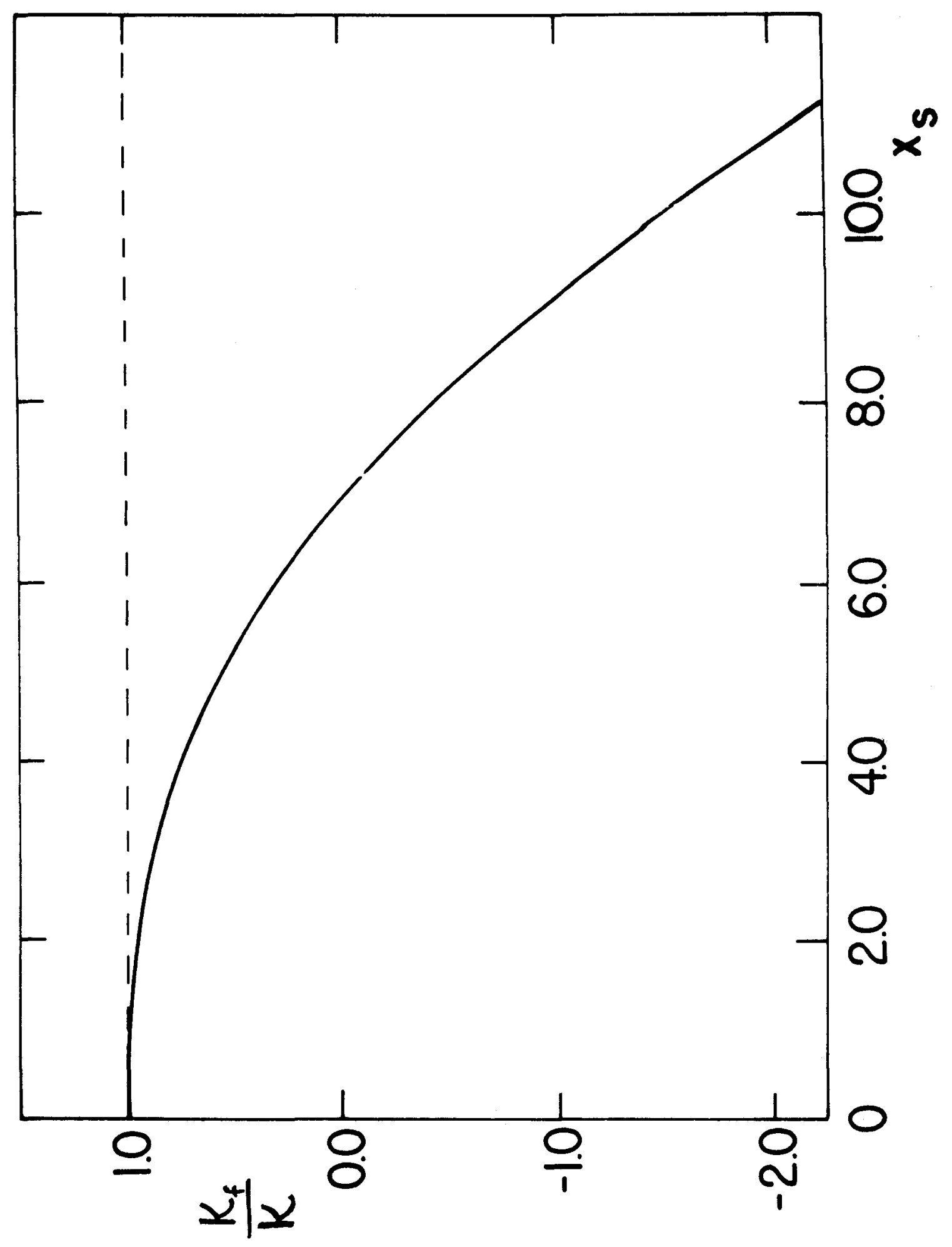

FIGURA 26 